\title{
Derivation of $\mathbf{F}=\mathbf{F}^{\mathrm{e}} \mathbf{F}^{\mathrm{p}}$ as the continuum limit of crystalline slip
}

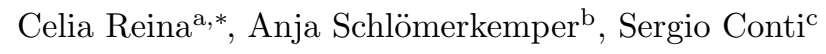 \\ ${ }^{a}$ Department of Mechanical Engineering and Applied Mechanics, University of Pennsylvania, Philadelphia, PA 19104, USA \\ ${ }^{b}$ Institut für Mathematik, Universität Würzburg, Emil-Fischer-Str. 40, 97074 Würzburg, Germany \\ ${ }^{c}$ Institut für Angewandte Mathematik, Universität Bonn, 53115 Bonn, Germany
}

\begin{abstract}
In this paper we provide a proof of the multiplicative kinematic description of crystal elastoplasticity in the setting of large deformations, i.e. $\mathbf{F}=\mathbf{F}^{\mathrm{e}} \mathbf{F}^{\mathrm{p}}$, for a two dimensional single crystal. The proof starts by considering a general configuration at the mesoscopic scale, where the dislocations are discrete line defects (points in the two-dimensional description used here) and the displacement field can be considered continuous everywhere in the domain except at the slip surfaces, over which there is a displacement jump. At such scale, as previously shown by two of the authors, there exists unique physically-based definitions of the total deformation tensor $\mathbf{F}$ and the elastic and plastic tensors $\mathbf{F}^{\mathrm{e}}$ and $\mathbf{F}^{\mathrm{p}}$ that do not require the consideration of any non-realizable intermediate configuration and do not assume any a priori relation between them of the form $\mathbf{F}=\mathbf{F}^{\mathrm{e}} \mathbf{F}^{\mathrm{p}}$. This mesoscopic description is then passed to the continuum limit via homogenization i.e., by increasing the number of slip surfaces to infinity and reducing the lattice parameter to zero. We show for two-dimensional deformations of initially perfect single crystals that the classical continuum formulation is recovered in the limit with $\mathbf{F}=\mathbf{F}^{\mathrm{e}} \mathbf{F}^{\mathrm{p}}$, $\operatorname{det} \mathbf{F}^{\mathrm{p}}=1$ and $\mathbf{G}=\operatorname{Curl} \mathbf{F}^{\mathrm{p}}$ the dislocation density tensor.
\end{abstract}

Keywords: Crystal plasticity, Finite kinematics, Dislocation density tensor, Homogenization

\section{Introduction}

Standard continuum models in the setting of large deformations are based on the kinematic assumption $\mathbf{F}=\mathbf{F}^{\mathrm{e}} \mathbf{F}^{\mathrm{p}}$, which decomposes the total deformation gradient $\mathbf{F}$ multiplicatively into the effective deformation induced by elastic and plastic mechanisms, $\mathbf{F}^{\mathrm{e}}$ and $\mathbf{F}^{\mathrm{p}}$ respectively. This approach was first introduced in the 1950's (Bilby et al., 1957; Kröner, 1959; Lee and Liu, 1967), although some of the underlying ideas may be found in earlier works, such as those by Eckart (1948) (see Sadik and Yavari (2015) for an in depth discussion on the origins of $\mathbf{F}=\mathbf{F}^{\mathrm{e}} \mathbf{F}^{\mathrm{p}}$ ); and its use has been very successful in many engineering applications (Simo, 1988; Ortiz and Repetto, 1999; Nemat-Nasser, 2004; Barton et al., 2013; Dassault Systèmes, 2012). However, the lack of a well-grounded justification for this decomposition has led to numerous debates in the literature. The heuristics behind the expression $\mathbf{F}=\mathbf{F}^{\mathrm{e}} \mathbf{F}^{\mathrm{p}}$ is based on the chain rule for the derivative of the deformation mapping $\mathbf{F}=D \boldsymbol{\varphi}$, when the elastic deformation of the system can be fully relaxed, leading to a purely plastic distortion. At the core of the problem lies the fact that dislocations, when present, physically couple the elastic and plastic field around them, preventing the existence of a physical configuration with associated deformation $\mathbf{F}^{\mathrm{e}}$ (or $\mathbf{F}^{\mathrm{p}}$ ) and thus exclusively elastic (or plastic). This inherent complexity has raised many issues, such as the physical meaning of the individual tensors $\mathbf{F}^{\mathrm{e}}$ or $\mathbf{F}^{\mathrm{p}}$, the existence (Casey and Naghdi, 1992; Deseri and Owen, 2002) and uniqueness (Nemat-Nasser, 1979; Lubarda and Lee, 1981; Zbib, 1993; Naghdi, 1990; Green and Naghdi, 1971; Casey and Naghdi, 1980; Mandel, 1973; Dafalias, 1987; Rice, 1971; Xiao et al., 2006) of the decomposition $\mathbf{F}=\mathbf{F}^{\mathrm{e}} \mathbf{F}^{\mathrm{p}}$, or the appropriate measure of dislocation content in the body (Bilby, 1955; Eshelby, 1956; Kröner, 1959; Fox, 1966; Willis, 1967; Acharya and Bassani, 2000; Cermelli and Gurtin, 2001) based on the incompatibilities of elastic or plastic component

*creina@seas.upenn.edu 
of the deformation. Other decompositions have also been proposed in the literature, some additive, typically in rate form (Truesdell (1955); Nemat-Nasser (1979); Zbib (1993); Pantelides (1994); Volokh (2013)), and others multiplicative $\left(\mathbf{F}=\mathbf{F}^{\mathrm{p}} \mathbf{F}^{\mathrm{e}}\right.$, Clifton (1972), Lubarda (1999); $\mathbf{F}$ based on a three factor decomposition Bammann and Johnson (1987); Lion (2000); Gerken and Dawson (2008); Henann and Anand (2009); Clayton et al. (2014); Casey (2015)).

A micromechanical definition of the different tensors $\mathbf{F}, \mathbf{F}^{\mathrm{e}}$ and $\mathbf{F}^{\mathrm{p}}$ has been recently provided (Reina and Conti, 2014) without any a priori assumption on the relationship between them and without recurring to any artificial intermediate configuration. These definitions are exclusively based on kinematic arguments and physical considerations, are uniquely determined from the microscopic displacement field, and lead to the expected multiplicative decomposition in the continuum limit in the absence of dislocations. From the previous definition of $\mathbf{F}^{\mathrm{p}}, \operatorname{Curl} \mathbf{F}^{\mathrm{p}}$ arises as the natural measure for the dislocation density tensor in the material.

Based on the above micromechanical definitions of $\mathbf{F}, \mathbf{F}^{\mathrm{e}}$ and $\mathbf{F}^{\mathrm{p}}$, we provide in this paper a rigorous proof of the continuum kinematic assumption $\mathbf{F}=\mathbf{F}^{\mathrm{e}} \mathbf{F}^{\mathrm{p}}$, with $\operatorname{det} \mathbf{F}^{\mathrm{p}}=1$, for a general dislocation and slip structure. Such generality is achieved in this work by considering microscopic elastoplastic displacement fields of finite energy that belong to a function space in which displacements are continuous everywhere in the domain except potentially at the slip surfaces over which there is a displacement jump. The continuum limit is then mathematically studied by letting the lattice parameter tend to zero and increasing the number of dislocations and slip surfaces to infinity. We show, for a two-dimensional deformation of an initially perfect single crystal, that the displacement field is indeed continuous in the limit, and that the limit of $\mathbf{F}$ coincides with the product of the limit of $\mathbf{F}^{\mathrm{e}}$ and $\mathbf{F}^{\mathrm{p}}$. The latter statement is highly non-trivial since limits and products do not generally commute. This complexity, combined with the non-linearity induced by the finite kinematic description, has so far limited most of the mathematical results of coarse-graining of dislocation ensembles to the linearized kinematic setting, see e.g. Conti and Ortiz (2005); Garroni et al. (2010) or De Luca et al. (2012), where the principle of superposition applies and the decomposition can be easily proven to be additive, i.e. $\varepsilon=\varepsilon^{\mathrm{e}}+\varepsilon^{\mathrm{p}}$, or to the case of well-separated dislocations (Scardia and Zeppieri, 2012; Müller et al., 2015). A more detailed understanding of the structure of pile-ups has been achieved under kinematic restrictions by Scardia et al. (2014), motion of dislocations in restricted models was studied by Blass et al. (2015); Alicandro et al. (2015). Even at the continuum scale, few mathematical results exist on finite elastoplasticity. Some examples can be found in Conti and Theil (2005), Mielke and Müller (2006); Mainik and Mielke (2009); Conti et al. (2011) and Hackl et al. (2012), the relation between linear and nonlinear context was elucidated by Mielke and Stefanelli (2013). A discrete model which does not require the existence of a reference configuration was developed by Luckhaus and Mugnai (2010).

Approaches closely related to the one of the present paper have already been used in order to improve the micromechanical understanding of the multiplicative decomposition, as in particular in the theory of structured deformations, see Del Piero and Owen (1993) and Deseri and Owen (2002), or in the averaging analyses of Davison (1995). One key idea in those papers is to explicitly account for microscopic slip activity, in that one considers deformations which are discontinuous on set of slip surfaces, and regular away from them. The total deformation gradient then consists of a smooth part, that can be interpreted as the elastic deformation tensor $\left(\mathbf{F}^{\mathrm{e}}\right)$, and a singular part, due to the presence of slips $(\mathbf{M})$, i.e. $\mathbf{F}=\mathbf{F}^{\mathrm{e}}+\mathbf{M}$. This additive decomposition, which does not resort to any artificial intermediate configuration, can be easily recast at $\mathbf{F}=\mathbf{F}^{\mathrm{e}} \mathbf{F}^{\mathrm{p}}$ with a suitable definition of $\mathbf{F}^{\mathrm{p}}$ as $\mathbf{F}^{\mathrm{p}}=\mathbf{I}+\left(\mathbf{F}^{\mathrm{e}}\right)^{-1} \mathbf{M}$. This approach bears significant similarities with the one of Reina and Conti (2014) used in the present paper, primarily the semicontinuous mesoscopic description, but differs in important aspects, as was discussed in Section 6.6 of Reina and Conti (2014), to which we refer for a more detailed comparison with the literature. In particular, our approach includes an explicit treatment of dislocations and a decomposition of slip processes into the composition of single slip maps, that delivers a bottom-up approach with independent definitions of the three deformation tensors, $\mathbf{F}, \mathbf{F}^{\mathrm{e}}$, and $\mathbf{F}^{\mathrm{p}}$, at the microstructural and continuum level. The mathematical approach is also quite different.

The paper is organized as follows. We begin in Section 2 by introducing the mathematical notation needed for the remainder of the text and reviewing the microscopic definitions of $\mathbf{F}, \mathbf{F}^{\mathrm{e}}$ and $\mathbf{F}^{\mathrm{p}}$ following the lines of Reina and Conti (2014). Next, in Section 3 we discuss the passage of the aforementioned quantities 

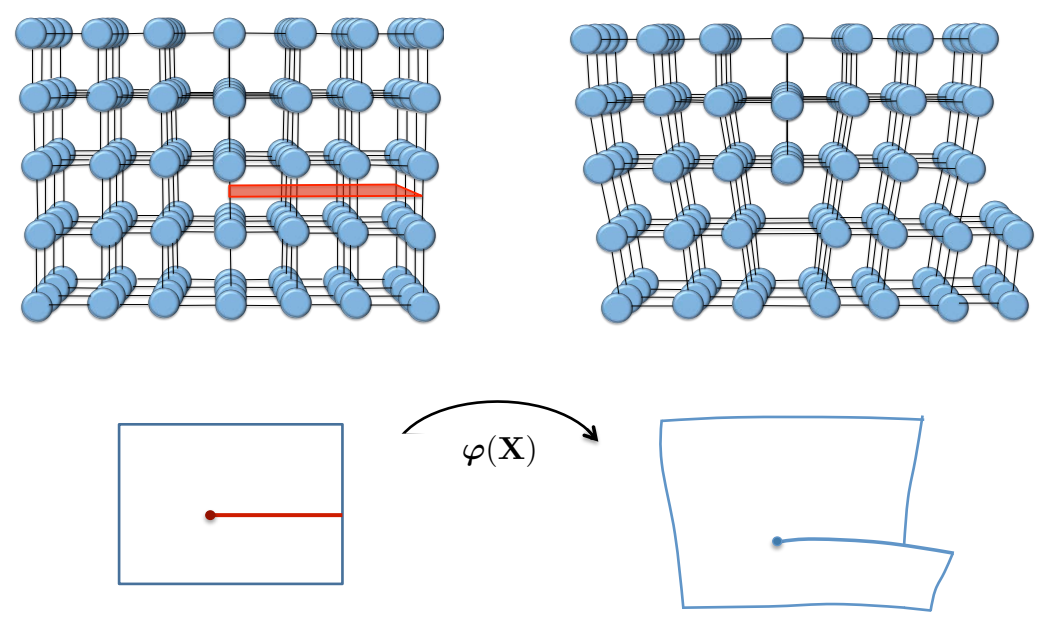

Figure 1: Elastoplastic deformation (right images) of a perfect crystal (left images) at the atomistic (top ${ }^{2}$ ) and mesoscopic scale (bottom) induced by the glide of an edge dislocation from the right surface of the domain till its center. The surface of displacement discontinuity is represented in red in the undeformed configuration and terminates at the dislocation point and the boundary of the domain.

to the continuum limit from a physical perspective, and provide some further guidance on notation in Section 4. This is followed by Sections 5 and 6 where the precise setting of the problem and the mathematical results are shown. The paper is then finalized with some conclusions in Section 7.

\section{Mesoscopic description of elastoplastic deformations}

The present study is exclusively concerned with two-dimensional elastoplastic deformations induced by dislocation glide (of edge type) and disregards other processes such as phase transformations, diffusion, void nucleation or appearance of microcracks. Mathematically, we describe these deformations with a semicontinuous Lagrangian formulation, where the final configuration $\varphi(\mathbf{X})$ of every material point $\mathbf{X}$ in the reference domain $\Omega \subset \mathbb{R}^{2}$, considered here to be a perfect crystal, is described via a deformation mapping $\varphi: \Omega \rightarrow \varphi(\Omega) \subset \mathbb{R}^{2}$. In particular, $\varphi$ is continuous everywhere in the domain, except at the area swept by the dislocations during their motion (lines in two dimensions), over which there is a displacement jump or discontinuity, cf. Fig. 1. These type of deformations belong to the space of special functions of bounded variation or $S B V$, cf. Evans and Gariepy (1991); Ambrosio et al. (2000); and we further require that $\varphi$ is one-to-one in order to avoid interpenetration of matter. The deformation gradient $\mathbf{F}$ is then defined as the distributional gradient of $\varphi$, which for SBV functions is a measure of the form

$$
\mathbf{F}=D \varphi=\nabla \varphi \mathcal{L}^{2}+\llbracket \varphi \rrbracket \otimes \mathbf{N} \mathcal{H}^{1}\lfloor\mathcal{J},
$$

where $\mathcal{L}^{2}$ denotes the Lebesgue measure (which measures area in the plane) and $\mathcal{H}^{1}$ the one-dimensional Hausdorff measure (which measures length). The measure $\mathbf{F}$ consists of an absolutely continuous part, $\nabla \boldsymbol{\varphi} \mathcal{L}^{2}$, and a singular part, $\llbracket \boldsymbol{\varphi} \rrbracket \otimes \mathbf{N} \mathcal{H}^{1}\lfloor\mathcal{J}$, that has its support over the slip lines or jump set $\mathcal{J}$, where $\mathbf{N}(\mathbf{X})$ is the unit normal at $\mathbf{X} \in \mathcal{J}$ and $\llbracket \varphi \rrbracket$ is the jump over the slip line, see below. The function $\nabla \boldsymbol{\varphi}$ is called the approximate differential of $\varphi$ and corresponds to the total deformation $\mathbf{F}$ at the points where no slip occurs and the deformation is thus purely elastic. As a result, $\nabla \varphi$ can be physically identified with the elastic deformation tensor

$$
\mathbf{F}^{\mathrm{e}}=\nabla \varphi
$$

\footnotetext{
${ }^{2}$ Reprinted from Publication 'Kinematic description of crystal plasticity in the finite kinematic framework: A micromechanical understanding of F=FeFp', Vol 67, Authors C. Reina and S. Conti, Page No. 43, Copyright (2014), with permission from Elsevier.
} 


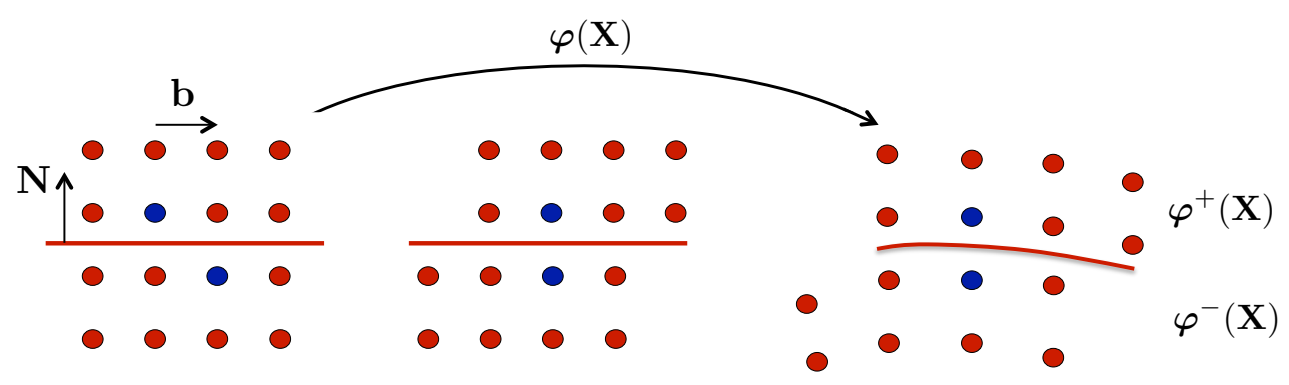

Figure 2: Atomic positions of a perfect crystal (left image) after a plastic deformation (middle image) and an elastoplastic deformation (right image) ${ }^{4}$.

The symbol $\nabla$ corresponds to the 'standard' gradient where the function is differentiable, in particular, only outside of the jump set. By definition, the full deformation gradient $\mathbf{F}$ has a vanishing (distributional) Curl. However, Curl $\nabla \varphi$ does not vanish in general, and the same occurs for the singular part of the deformation gradient. We note that an additive decomposition of the deformation gradient of the form of (2.1), with the first term being identified as the elastic deformation tensor, has been previously considered in the mechanics literature based on local volumetric averages and application of the divergence theorem over continuous regions of the domain (Davison, 1995). An analogous additive decomposition is also found in the theory of structured deformations, introduced by Del Piero and Owen (1993, 2004), cf. Deseri and Owen (2002).

For its part, $\llbracket \varphi \rrbracket(\mathbf{X})=\varphi^{+}(\mathbf{X})-\varphi^{-}(\mathbf{X})$ is the displacement jump at point $\mathbf{X}$ in the slip line, where the + side is the one indicated by the normal $\mathbf{N}$ to the line at point $\mathbf{X}$. Without further restrictions on $\llbracket \varphi \rrbracket$ and $\mathcal{J}$, the class of deformation mappings considered thus far can also model processes such as cavitation, crack opening or interpenetration of matter. A careful analysis of the kinematics of slip in crystalline materials, cf. Reina and Conti (2014), indicates that jump sets in two dimensions necessarily consist of an ensemble of straight segments in the reference configuration that terminate at dislocation points or exit the domain, cf. Fig. 1; and that the displacement jump for single slip satisfies, cf. Fig. 2,

$$
\begin{aligned}
& \varphi^{-}(\mathbf{X})=\varphi^{+}(\mathbf{X}-\mathbf{b}), \quad \forall \mathbf{X}, \mathbf{X}-\mathbf{b} \in \mathcal{J}, \\
& \varphi^{+}(\mathbf{X})=\varphi^{-}(\mathbf{X}+\mathbf{b}), \quad \forall \mathbf{X}, \mathbf{X}+\mathbf{b} \in \mathcal{J},
\end{aligned}
$$

where $\mathbf{b}$ is called the Burgers vector and represents the interatomic distance in the direction of slip. We remark that the two conditions in (2.3) are equivalent when $\mathbf{X}, \mathbf{X}-\mathbf{b}, \mathbf{X}+\mathbf{b} \in \mathcal{J}$.

The jump set $\mathcal{J}$ and the previously introduced vector $\mathbf{b}$ for a single slip system, clearly characterize the plastic deformation with quantities independent of the elastic distortion. Indeed, if we consider for instance a plastic simple shear deformation, such as $\varphi_{1}$ in Fig. 3, the total deformation is purely plastic and thus $D \varphi_{1}$ is equal to $\mathbf{F}^{\mathrm{p}}$, and can be expressed, cf. Eq. (2.1), as

$$
\mathbf{F}=D \varphi_{1}=\mathbf{F}^{\mathrm{p}}=\mathbf{I} \mathcal{L}^{2}+\left|\mathbf{b}_{1}\right| \mathbf{e}_{1} \otimes \mathbf{e}_{2} \mathcal{H}^{1}\lfloor\mathcal{J}
$$

These deformations, which corresponds to rigid blocks sliding over each other, are called two-level shears in the context of generalized deformations, see for example the discussion in Del Piero and Owen (2004).

The situation becomes, however, more complex for the composition of two simple shears where slip lines intersect, cf. Fig. 3 and Reina and Conti (2014), the plastic deformation tensor is uniquely given from Eq. (2.1) as

$$
\mathbf{F}=D\left(\boldsymbol{\varphi}_{2} \circ \boldsymbol{\varphi}_{1}\right)=\mathbf{F}^{\mathrm{p}}=\mathbf{I} \mathcal{L}^{2}+\left|\mathbf{b}_{1}\right| \mathbf{e}_{1} \otimes \mathbf{e}_{2} \mathcal{H}^{1}\left\lfloor\mathcal{J}_{1}+\left|\mathbf{b}_{2}\right| \mathbf{e}_{2} \otimes \mathbf{e}_{1} \mathcal{H}^{1}\left\lfloor\mathcal{J}_{2}+\left|\mathbf{b}_{2}\right| \mathbf{e}_{2} \otimes \mathbf{e}_{2} \mathcal{H}^{1}\left\lfloor\mathcal{J}_{\text {kink }} .\right.\right.\right.
$$

\footnotetext{
${ }^{4}$ Reprinted from Publication 'Kinematic description of crystal plasticity in the finite kinematic framework: A micromechanical understanding of F=FeFp', Vol 67, Authors C. Reina and S. Conti, Page No. 45, Copyright (2014), with permission from Elsevier.
} 


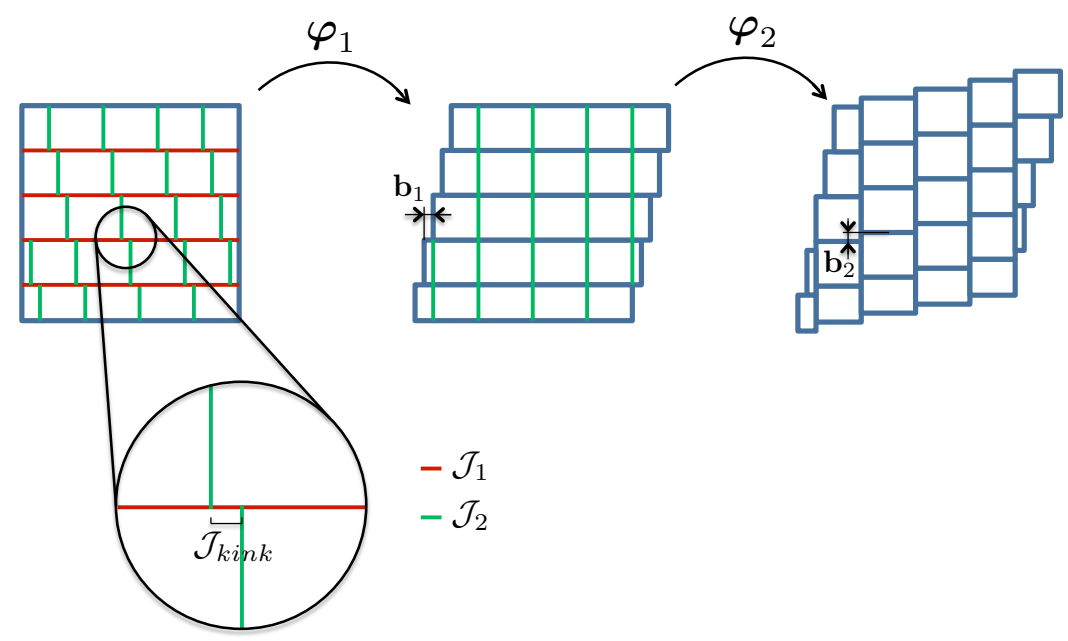

Figure 3: Sequence of plastic deformations involving two orthogonal slip systems. The zoom in the reference configuration is used to label the segments composing the jump set.

The last term in Eq. (2.5) relates to the kink that results from the pullback of the second slip lines into the reference configuration and provides the non-commutative term in the finite kinematic formulation. In other words, the pullback is performed in opposite order in which the slip occurred and therefore the resulting kink term is dependent on whether the slip is first performed in the horizontal or vertical direction.

The above results can be easily generalized for an arbitrary sequence of $N_{s}$ dislocation-free plastic slips, not necessarily homogeneous or belonging to orthogonal slip systems. In that case, $\varphi$ can be written as $\varphi=\varphi_{N_{s}} \circ \ldots \circ \varphi_{\nu} \circ \ldots \circ \varphi_{1}$, where $\mathcal{J}_{\varphi_{\nu}}$ (jump set associated to $\varphi_{\nu}$ ) is an ensemble of parallel straight infinite lines with normal $\mathbf{N}_{\nu}$ and

$$
D \boldsymbol{\varphi}_{\nu}=\mathbf{I} \mathcal{L}^{2}+\mathbf{b}_{\nu} \otimes \mathbf{N}_{\nu} \mathcal{H}^{1}\left\lfloor\mathcal{J}_{\varphi_{\nu}}, \quad \mathbf{b}_{\nu} \cdot \mathbf{N}_{\nu}=0, \quad \mathbf{b}_{\nu} \in \mathbb{R}^{2}\right. \text { constant on each line. }
$$

The total plastic deformation tensor associated to $\varphi$ can then be written as

$$
\mathbf{F}^{\mathrm{p}}=\mathbf{I} \mathcal{L}^{2}+\sum_{j} \mathbf{b}_{j} \otimes \mathbf{N}_{j} \mathcal{H}^{1}\left\lfloor\mathcal{J}_{j}\right.
$$

where the total jump set $\mathcal{J}$ in the reference configuration consists of the union of straight segments $\mathcal{J}_{j}$ (appropriately including all kinks), each of which has an associated Burgers vector $\mathbf{b}_{j}$. In general, as was the case for the composition of two simple shears, $\mathbf{b}_{j} \cdot \mathbf{N}_{j}$ is not necessarily zero. Equation (2.7) remains of course valid if an elastic deformation is superposed to the previous plastic distortion, i.e. $\varphi=\varphi^{\mathrm{e}} \circ \varphi^{\mathrm{p}}, \mathbf{F}^{\mathrm{p}}=D \varphi^{\mathrm{p}}$. In such case, by construction, $\mathbf{F}=D \varphi=D\left(\varphi^{\mathrm{e}} \circ \varphi^{\mathrm{p}}\right)=\nabla\left(\boldsymbol{\varphi}^{\mathrm{e}} \circ \varphi^{\mathrm{p}}\right) \mathcal{L}^{2}+\left(\boldsymbol{\varphi}^{\mathrm{e}}\left(\boldsymbol{\varphi}^{\mathrm{p}+}\right)-\varphi^{\mathrm{e}}\left(\boldsymbol{\varphi}^{\mathrm{p}-}\right)\right) \otimes \mathbf{N} \mathcal{H}^{1}\lfloor\mathcal{J}$, $\mathbf{F}^{\mathrm{e}}=\nabla \varphi=\nabla\left(\varphi^{\mathrm{e}} \circ \varphi^{\mathrm{p}}\right)$, and, if $\mathbf{F}^{\mathrm{e}}$ is sufficiently smooth one obtains that for compatible domains $\mathbf{F}=$ $\mathbf{F}^{\mathrm{e}} \mathbf{F}^{\mathrm{p}}+\mathcal{O}\left(|\mathbf{b}|^{2}\right)$, cf. Reina and Conti (2014).

In a general elastoplastic deformation, dislocations are present in the body, coupling the elastic and plastic field. They render impossible a global decomposition of the total deformation mapping into a purely plastic distortion and a subsequent elastic deformation, i.e. $\varphi \neq \varphi^{\mathrm{e}} \circ \varphi^{\mathrm{p}}$, thus complicating, in principle, the physical definition of the global plastic deformation tensor $\mathbf{F}^{\mathrm{p}}$. However, at this mesoscopic scale where the dislocations are individually resolved, subdomains away from the dislocations are, by construction, defect free and it is therefore possible to obtain in each of them a decomposition of the form $\varphi=\varphi^{\mathrm{e}} \circ \varphi^{\mathrm{p}}$ and an associated $\mathbf{F}^{\mathrm{p}}$ given by $D \varphi^{\mathrm{p}}$, as in Eq. (2.7). It will be shown in Section 5 that $\mathbf{F}^{\mathrm{p}}$ is uniquely defined from $\varphi$ regardless of the potentially many decompositions of the form $\varphi^{\mathrm{e}} \circ \varphi^{\mathrm{p}}$ in each of these subdomains, thus providing a global definition of $\mathbf{F}^{\mathrm{p}}$ almost everywhere in the domain (except at the dislocation cores and close to the boundary). 


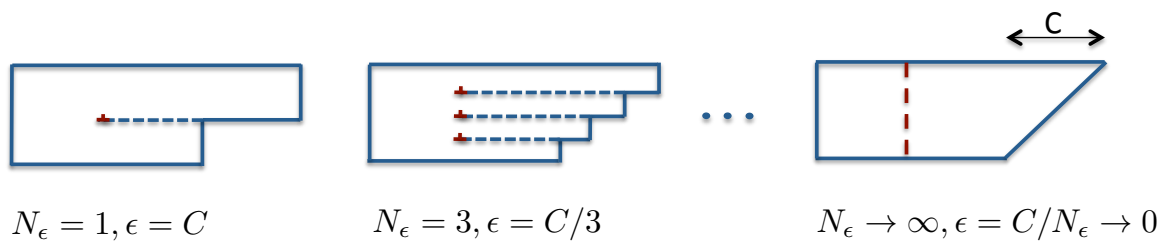

Figure 4: Sequence of elastoplastic deformations as the lattice parameter $\epsilon$ tends to zero, and the number of dislocations $N_{\epsilon}$ and length of the jump set $|\mathcal{J}|$ tends to infinity.

It can therefore be concluded that $\mathbf{F}, \mathbf{F}^{\mathrm{e}}$ and $\mathbf{F}^{\mathrm{p}}$ are uniquely defined from the mesoscopic deformation mapping $\varphi$ in general elastoplastic deformations induced by dislocation glide, and are of the form

$$
\begin{aligned}
& \mathbf{F}=D \boldsymbol{\varphi}=\nabla \boldsymbol{\varphi} \mathcal{L}^{2}+\sum_{j} \llbracket \varphi \rrbracket \otimes \mathbf{N}_{j} \mathcal{H}^{1}\left\lfloor\mathcal{J}_{j},\right. \\
& \mathbf{F}^{\mathrm{e}}=\nabla \boldsymbol{\varphi}, \\
& \mathbf{F}^{\mathrm{p}}=\mathbf{I} \mathcal{L}^{2}+\sum_{j} \mathbf{b}_{j} \otimes \mathbf{N}_{j} \mathcal{H}^{1}\left\lfloor\mathcal{J}_{j} .\right.
\end{aligned}
$$

As will be shown in the following sections, the precise definition of $\mathbf{F}^{\mathrm{p}}$ inside the dislocation cores will be irrelevant in the continuous limit. However, for concreteness, the above definition for $\mathbf{F}^{\mathrm{p}}$ is extended to the interior of the cores, with a constant Burgers vector for each segment terminating at the dislocation point (this will be made precise in Section 5). This choice of $\mathbf{F}^{\mathrm{p}}$ associates to each segment composing the jump set a constant Burgers vector, and as derived analytically in Reina and Conti (2014), Curl $\mathbf{F}^{\mathrm{p}}=\sum_{k=1}^{N_{\epsilon}} \mathbf{b}_{k} \delta_{\mathbf{X}_{k}}$, where $\left\{\mathbf{X}_{k}\right\}$ is the ensemble of $N_{\epsilon}$ dislocation points and $\mathbf{b}_{k}$ the corresponding Burgers vector (sum of the Burgers vector of the slip lines terminating at the dislocation point, with the appropriate sign). In other words, $\mathbf{G}=$ Curl $\mathbf{F}^{\mathrm{p}}$ exactly measures the dislocation content in the body and is therefore an appropriate definition of the dislocation density tensor.

\section{Scaling towards the continuum limit}

Ultimately, we are interested in the description of the elastoplastic deformation in the continuum limit. To that regard, we consider sequences of deformations, see Fig. 4, in which each element of the sequence is characterized by the parameter $\epsilon$ that measures the normalized lattice parameter (ratio between the shortest atomic distance and the characteristic length of the body). In particular, each deformation mapping will be denoted as $\boldsymbol{\varphi}_{\epsilon}$, and its jump set and the Burgers vector of each segment as $\mathcal{J}_{\epsilon}=\sum_{j} \mathcal{J}_{\epsilon j}$ and $\mathbf{b}_{\epsilon j}$ respectively. Similarly, the different deformation tensors, as given by Eqs. (2.8), will be denoted as $\mathbf{F}_{\epsilon}, \mathbf{F}_{\epsilon}^{\mathrm{e}}$ and $\mathbf{F}_{\epsilon}^{\mathrm{p}}$. Furthermore, by crystallographic arguments, the number of slip systems is finite and bounded by some fixed $N_{s} ;\left|\mathbf{b}_{\epsilon j}\right| \leq n \epsilon$ for some fixed $n>0$; and planes of the same slip system are separated by a distance that is equal to or larger than $\epsilon$. From the latter, it is implied that, cf. Fig. 5,

$$
\mathcal{H}^{1}\left(\mathcal{J}_{\epsilon} \cap B_{r}(\mathbf{X})\right) \leq A \frac{r^{2}}{\epsilon}
$$

for some $A$ that depends on $N_{s}$. In the following, the subindex $\epsilon$ will be omitted for the jump set $\mathcal{J}_{\epsilon}$ since the limiting deformation mapping will be continuous, and therefore $\mathcal{J}$ is unequivocally associated to an element of the sequence. Similarly, no subindex $\epsilon$ will be used to denote the dislocation points $\mathbf{X}_{k}$ along the sequence.

The continuum limit is then attained as $\epsilon$ vanishes, and the macroscopic kinematic quantities $\varphi, \mathbf{F}, \mathbf{F}^{\mathrm{e}}$ and $\mathbf{F}^{\mathrm{p}}$ are uniquely defined as the limit of the corresponding mesoscopic quantities, i.e.

$$
\varphi_{\epsilon} \rightarrow \varphi, \quad \mathbf{F}_{\epsilon} \rightarrow \mathbf{F}, \quad \mathbf{F}_{\epsilon}^{\mathrm{e}} \rightarrow \mathbf{F}^{\mathrm{e}}, \quad \mathbf{F}_{\epsilon}^{\mathrm{p}} \rightarrow \mathbf{F}^{\mathrm{p}}
$$




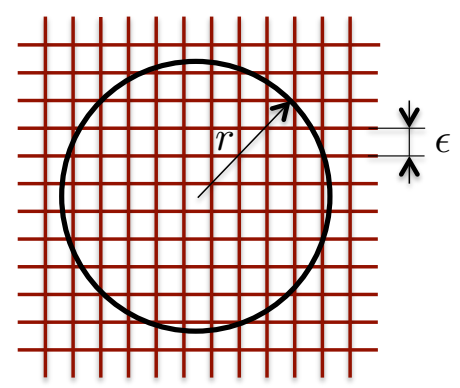

Figure 5: Jump sets corresponding to the same slip system are separated by a distance of at least $\epsilon$. In the case of two orthogonal slip systems, the maximum length of slip lines contained in a ball of radius $r$ is then proportional to $\frac{r^{2}}{\epsilon}$.

as $\epsilon \rightarrow 0$ in the appropriate topology. This perspective thus delivers a well defined $\mathbf{F}^{\mathrm{e}}$ and $\mathbf{F}^{\mathrm{p}}$, that do not invoke any macroscopic unloading path, which in general does not exist. Only at the mesoscopic scale, as discussed in the previous section, a relaxation process (or decomposition of the deformation mapping into a physically realizable elastic and plastic deformation) is defined everywhere except at the dislocation cores.

In analogy to a zoom out process in a real material, the number of dislocations $N_{\epsilon}$ and the total length of the jump set $|\mathcal{J}|$ tend, potentially, to infinity as $\epsilon \rightarrow 0$. Equation (3.1) directly implies

$$
\mathcal{H}^{1}(\mathcal{J}) \epsilon \leq C^{*},
$$

for some $C^{*}$, and thus $\mathbf{F}_{\epsilon}^{\mathrm{p}}$ is at most a finite deformation, as desired. We further assume that the number of dislocations $N_{\epsilon}$ satisfies

$$
N_{\epsilon} \epsilon \leq C,
$$

for some $C$. This scaling allows the formation of dislocation walls and prevents the clustering of infinitely many dislocations at one macroscopic material point, as is commonly observed in experiments. To further support the scaling given by Eq. (3.4), consider an ensemble of identical dislocations positioned over a finite and fixed subdomain with a regular square pattern, and denote by $\delta$ the distance between the dislocations. The remainder of the domain is considered dislocation-free along the sequence. The total amount of dislocations $N_{\epsilon}$ is then proportional to $\frac{1}{\delta^{2}}$ and it will tend to infinity as $\delta$ tends to zero. The elastic deformation away form the cluster (on the dislocation-free region) scales as $\frac{\epsilon}{r \delta^{2}}$ in the linearized kinematic framework, where $r$ is the distance to the cluster. The energy density is then proportional to $\left(\frac{\epsilon}{r \delta^{2}}\right)^{2}$, and the total elastic energy on the non-dislocated region scales as $\left(\frac{\epsilon}{\delta^{2}}\right)^{2}$. Such an energy will then be finite only if $N_{\epsilon} \sim \frac{1}{\delta^{2}} \leq \frac{C}{\epsilon}$.

In the following sections, we will show that the macroscopic quantities $\mathbf{F}, \mathbf{F}^{\mathrm{e}}$ and $\mathbf{F}^{\mathrm{p}}$ defined in (3.2) are related via the standard multiplicative decomposition $\mathbf{F}=\mathbf{F}^{\mathrm{e}} \mathbf{F}^{\mathrm{p}}$ with $\operatorname{det} \mathbf{F}^{\mathrm{p}}=1$, that $\boldsymbol{\varphi}$ is continuous and that $\mathbf{G}=$ Curl $\mathbf{F}^{\mathrm{p}}$ represents the continuum dislocation density tensor when expressed in the reference configuration.

\section{Notation}

The notation used in the following will be, when possible, the standard notation used in continuum mechanics. In particular, vectors and tensors are denoted with boldface symbols (the order of the tensor will be clear from the context), and scalars are written in standard font. Similarly $d X=d \mathcal{L}^{2}$ denotes the differential area in the reference configuration and $d \mathbf{X}$ a differential vector, so that

$$
\int_{\mathbf{X}_{1}}^{\mathbf{X}_{2}} \mathbf{f}(\mathbf{X}) \cdot d \mathbf{X}=\int_{0}^{1} \mathbf{f}\left(\mathbf{X}_{1}+t\left(\mathbf{X}_{2}-\mathbf{X}_{1}\right)\right) \cdot\left(\mathbf{X}_{2}-\mathbf{X}_{1}\right) d t
$$



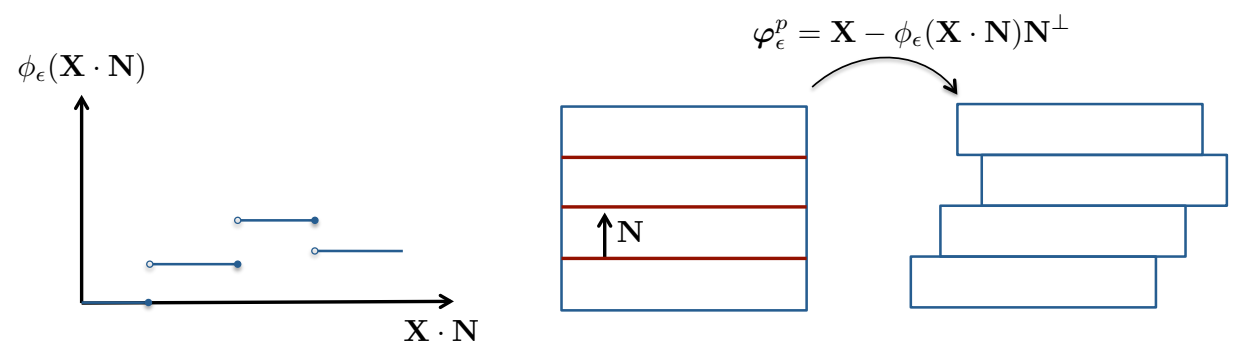

Figure 6: Example of a single slip plastic deformation $\varphi_{\epsilon}^{\mathrm{p}}(\mathbf{X})=\mathbf{X}-\phi_{\epsilon}(\mathbf{X} \cdot \mathbf{N}) \mathbf{N}^{\perp}$. The value of $\phi_{\epsilon}$ at the jump point is arbitrary, but uniquely defined, so that $\varphi_{\epsilon}^{\mathrm{p}}$ is bijective.

The scalar product between two vectors is denoted as $\mathbf{f} \cdot \mathbf{g}$, the full contraction between two tensors as $\mathbf{A}: \mathbf{B}$, the matrix vector multiplication with no symbol $\mathbf{A g}$ and $\otimes$ is used to denote the dyadic product between two vectors $\mathbf{f} \otimes \mathbf{g}$. Furthermore, $|\cdot|$ is used to denote the Euclidean norm of the scalar, vector or tensor that the symbol encloses. For sets, we use the notation $|A|=\mathcal{H}^{1}(A)$ for length and $|A|=\mathcal{L}^{2}(A)$ for area, where the dimension of the set $A$ is clear from the context.

The spaces that will be used in the following are the space of Radon measures $\mathcal{M}_{\text {loc }}$, the subspace of bounded measures $\mathcal{M}$, the $L^{p}$ spaces, the space of functions of bounded variation $B V$ and the space of special functions of bounded variations $S B V$. Further details on these spaces may be found in Ambrosio et al. (2000), Evans and Gariepy (1991) and Adams and Fournier (2003).

The deformation mappings $\varphi_{\epsilon}$ and $\varphi_{\epsilon}^{\mathrm{p}}$ will be considered uniquely defined at the jump set, by arbitrarily choosing the value at either side of the jump. With these considerations in mind, both mappings are bijective, see Fig. 6. In the compatible regions of the domain, where $\varphi_{\epsilon}$ is of the form $\varphi_{\epsilon}=\varphi_{\epsilon}^{\mathrm{e}} \circ \varphi_{\epsilon}^{\mathrm{p}}$, the choice of $\varphi_{\epsilon}, \varphi_{\epsilon}^{\mathrm{p}}$ and the domain of $\varphi_{\epsilon}^{\mathrm{e}}$ need to be consistent with each other. Similar considerations apply for $\mathbf{F}_{\epsilon}^{\mathrm{e}}(\mathbf{X})=\nabla\left(\boldsymbol{\varphi}_{\epsilon}^{\mathrm{e}} \circ \varphi_{\epsilon}^{\mathrm{p}}\right)=\tilde{\nabla} \boldsymbol{\varphi}_{\epsilon}^{\mathrm{e}} \circ \boldsymbol{\varphi}_{\epsilon}^{\mathrm{p}}=\tilde{\mathbf{F}}_{\epsilon}^{\mathrm{e}} \circ \boldsymbol{\varphi}_{\epsilon}^{\mathrm{p}}$ in the compatible region, where the tilde is used to denote quantities in the image of $\varphi_{\epsilon}^{\mathrm{p}}$, i.e. $\tilde{\mathrm{X}}=\varphi_{\epsilon}^{\mathrm{p}}(\mathrm{X})$.

Finally, for ease of notation, the subindex $k$ will be reserved for dislocations, $j$ for segments of the jump set in the reference configuration, $\nu$ for segments of the jump set of a single slip system in the appropriate compatible plastic configuration, and $l$ for domains in the compatible regions away from the dislocation cores.

\section{Setting of the problem}

Let $\Omega \subset \mathbb{R}^{2}$ be an open bounded set with Lipschitz boundary ${ }^{5}$, describing the reference configuration of a defect-free crystalline material. For every $\epsilon>0$, we consider deformation mappings $\varphi_{\epsilon}: \Omega \rightarrow \varphi_{\epsilon}(\Omega) \subset \mathbb{R}^{2}$, $\varphi_{\epsilon} \in X_{\epsilon}$ defined later in (5.4), with finite energy, described by the following functional

$$
E_{T \epsilon}\left(\boldsymbol{\varphi}_{\epsilon}\right)=E_{\epsilon}\left(\boldsymbol{\varphi}_{\epsilon}\right)-\int_{\Omega} \mathbf{f} \cdot \boldsymbol{\varphi}_{\epsilon} d X-\int_{\partial \Omega} \mathbf{t} \cdot \boldsymbol{\varphi}_{\epsilon} d \mathcal{H}^{1}
$$

where $\mathbf{f}$ are body forces, $\mathbf{t}$ are the prescribed tractions at the boundary (body forces and tractions are assumed to be a system of zero net force and momentum for static equilibrium) and

$$
E_{\epsilon}\left(\boldsymbol{\varphi}_{\epsilon}\right)=\int_{\Omega \backslash \bigcup_{k=1}^{N_{\epsilon}} B_{c \epsilon}\left(\mathbf{X}_{k}\right)} W^{\mathrm{e}}\left(\mathbf{F}_{\epsilon}^{\mathrm{e}}\right) d X+\alpha \int_{\bigcup_{k=1}^{N_{\epsilon}} B_{c \epsilon}\left(\mathbf{X}_{k}\right)} W^{\mathrm{e}}\left(\mathbf{F}_{\epsilon}^{\mathrm{e}}\right) d X+\beta\left|D \mathbf{F}_{\epsilon}^{\mathrm{e}}\right|(\Omega),
$$

where $\alpha, \beta>0$ are material parameters, $\mathbf{F}_{\epsilon}^{\mathrm{e}}$ is the elastic deformation tensor and $B_{c \epsilon}\left(\mathbf{X}_{k}\right)$ denotes the dislocation cores. We note that proofs will only use the coercivity of $E_{\epsilon}$, and thus additional nonnegative

\footnotetext{
5i.e., with no 'cusps' (see, for instance, Evans and Gariepy (1991) for a rigorous definition of Lipschitz boundary)
} 
terms may be added to the energy without affecting the results. In particular, a term dependent on the plastic deformation tensor $\mathbf{F}_{\epsilon}^{\mathrm{p}}$ is physically justified to provide an energetic compromise between elastic and plastic mechanisms for achieving a deformation compliant with the external loads.

The elastic energy functional given by Eq. (5.2) is composed of three terms. The first two terms represent the elastic energy outside and inside the dislocation cores respectively, where each core is considered to be a ball of radius $c \epsilon$, around the dislocation points $\mathbf{X}_{k}$. As usual, we consider continuous elastic energy densities $W^{\mathrm{e}}\left(\mathbf{F}_{\epsilon}^{\mathrm{e}}\right)$ with quadratic growth, i.e.,

$$
\frac{1}{C_{L}}\left|\mathbf{F}^{\mathrm{e}}\right|^{2}-C_{L} \leq W^{\mathrm{e}}\left(\mathbf{F}^{\mathrm{e}}\right) \leq C_{U}\left|\mathbf{F}^{\mathrm{e}}\right|^{2}+C_{U}
$$

with $C_{L}, C_{U}>0$. Continuum mechanics is expected to fail in the core, and the parameter $\alpha$ provides the necessary rescaling of the dislocation core energy to match its physical value, as given, for instance, by atomistic simulations. However, the value of $\alpha$ will be irrelevant for the proofs and may be set to one if desired. For its part, the last term of Eq. (5.2) represents the total variation of the measure $D \mathbf{F}_{\epsilon}^{\mathrm{e}}$ over the set $\Omega$. It is mainly introduced for mathematical reasons, and it is needed to obtain $\mathbf{F}_{\epsilon} \approx \mathbf{F}_{\epsilon}^{\mathrm{e}} \mathbf{F}_{\epsilon}^{\mathrm{p}}$ in the domain, see Proposition 6.1. It can be physically interpreted as a non-local elastic deformation term that prevents, for instance, the formation of infinitely thin laminated microstructures with alternate elastic deformations. This type of energy terms are common in the so-called 'gradient elasticity' theories (Aifantis, 2009).

The function space $X_{\epsilon}$, defined below, includes the kinematic restrictions of slip described in Section 2, the crystallographic conditions and scalings discussed in Section 3, and considers two additional constraints that attend to mathematical simplicity. In particular, dislocations are considered to be separated from each other and from the boundary of the domain by a distance of at least several atomic spacings, and the sequence of potentially activated plastic slip systems will be considered fixed with all slip systems distinct. Although this last assumption may appear artificial and indeed does not account for the full set of compatible elastoplastic deformations that a material may suffer (a weaving structure common in textiles would be out of this scope), we will show later in Lemma 5.6 that an arbitrary plastic deformation tensor may be written as the composition of three simple shears with prescribed slip direction and order of activation. The domain is then separated into the dislocation cores $B_{c \epsilon}\left(\mathbf{X}_{k}\right)$, where the Burgers vector is spread over a ball of radius $m \epsilon<c \epsilon$ to remove the elastic singularity, and $\Omega \backslash \bigcup_{k=1}^{N_{\epsilon}} B_{m \epsilon}\left(\mathbf{X}_{k}\right)$, where the deformation mapping is compatible. The regions of overlap, $B_{c \epsilon}\left(\mathbf{X}_{k}\right) \backslash B_{m \epsilon}\left(\mathbf{X}_{k}\right)$, are locally compatible and allow a natural extension of the definition of $\mathbf{F}^{\mathrm{p}}$ everywhere inside the core region. As will be shown in the following, the specific details of the deformation inside each of these cores as well as the definition of $\mathbf{F}^{\mathrm{p}}$ in these regions will be irrelevant in the continuum limit, justifying the use of specific functional forms for the deformation in the cores.

In the following we give a precise definition of the space $X_{\epsilon}$. It depends on three global constants independent of $\epsilon$, namely, the number of distinct slip systems $N_{s}$, the normalized maximum length of the Burgers vector for an individual slip $\left|\mathbf{b}_{\epsilon} / \epsilon\right| \leq n^{\prime}$, and the scaling associated to the number of dislocations $C$, cf. Eq. (3.4). We define

$$
X_{\epsilon}=\left\{\boldsymbol{\varphi}_{\epsilon} \in S B V\left(\Omega ; \mathbb{R}^{2}\right): \boldsymbol{\varphi}_{\epsilon}, \mathbf{F}_{\epsilon}^{\mathrm{e}}, \mathbf{F}_{\epsilon}^{\mathrm{p}} \text { satisfy conditions }(\mathrm{C} 1)-(\mathrm{C} 4)\right\}
$$

for some global constants $n^{\prime}, C, N_{s}>0$ and some $N_{s}$ global unit vectors $\mathbf{N}_{1}, \ldots, \mathbf{N}_{\nu}, \ldots, \mathbf{N}_{N_{s}}$, all distinct. The constants $c, m, L, n>0$ will be defined below from $n^{\prime}, C, N_{s}$.

(C1) There are $N_{\epsilon}$ dislocation points $\left(\mathbf{X}_{k}\right)_{k}$, with

$$
N_{\epsilon} \leq \frac{C}{\epsilon}
$$

which describe the dislocations. They are well-separated in the sense that

$$
\begin{aligned}
& \left|\mathbf{X}_{k_{1}}-\mathbf{X}_{k_{2}}\right|>3 c \epsilon, \quad \forall k_{1} \neq k_{2}, \\
& \operatorname{dist}\left(\mathbf{X}_{k}, \partial \Omega\right)>2 c \epsilon, \quad \forall k=1, \ldots, N_{\epsilon},
\end{aligned}
$$




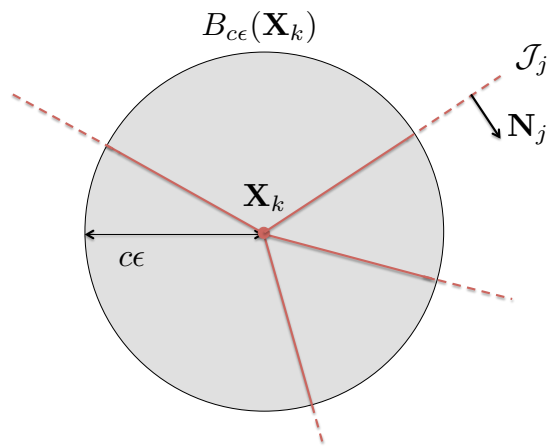

Figure 7: Example of a dislocation core $B_{c \epsilon}\left(\mathbf{X}_{k}\right)$. The dislocation is centered at $\mathbf{X}_{k}$ and is the boundary of a finite number of slip lines $\mathcal{J}_{j}, 1 \leq j \leq N_{d k} \leq N_{s}$.

where $c=m+2 L^{2}$, with $m=2 N_{s} n^{\prime}$ and $L>0$ as defined later in Lemma 5.1. The value of $m$ is discussed in (C3) and the value of $c$ results from Lemma 5.3.

The jump set in each dislocation core $\mathbf{X}_{k}, \mathcal{J} \cap B_{c \epsilon}\left(\mathbf{X}_{k}\right)$, consists of $N_{d k} \leq N_{s}$ distinct straight segments joining $\mathbf{X}_{k}$ with a point in $\partial B_{c \epsilon}\left(\mathbf{X}_{k}\right)$.

(C2) For every convex domain $\omega \subset \mathbb{R}^{2} \backslash \bigcup_{k=1}^{N_{\epsilon}} B_{m \epsilon}\left(\mathbf{X}_{k}\right), \varphi_{\epsilon}$ is a compatible deformation in $\omega \cap \Omega$, in the sense that there are two maps $\varphi_{\epsilon}^{\mathrm{e}}$ and $\varphi_{\epsilon}^{\mathrm{p}} \operatorname{such}$ that

$$
\boldsymbol{\varphi}_{\epsilon}(\mathbf{X})=\left(\varphi_{\epsilon}^{\mathrm{e}} \circ \varphi_{\epsilon}^{\mathrm{p}}\right)(\mathbf{X}) \quad \forall \mathbf{X} \in \omega \cap \Omega,
$$

where $\varphi_{\epsilon}^{\mathrm{e}}: \mathbb{R}^{2} \rightarrow \mathbb{R}^{2}$ is Lipschitz continuous and one-to-one and $\varphi_{\epsilon}^{\mathrm{p}}: \mathbb{R}^{2} \rightarrow \mathbb{R}^{2}$ can be written as the composition of $N_{s}$ single slip deformations, $\varphi_{\epsilon}^{\mathrm{p}}=\varphi_{\epsilon, N_{s}}^{\mathrm{p}} \circ \ldots \circ \varphi_{\epsilon, \nu}^{\mathrm{p}} \circ \ldots \circ \varphi_{\epsilon, 1}^{\mathrm{p}}$, where each $\varphi_{\epsilon, \nu}^{\mathrm{p}}$ is of the form

$$
\varphi_{\epsilon, \nu}^{\mathrm{p}}(\mathbf{X})=\mathbf{X}-\phi_{\epsilon, \nu}\left(\mathbf{X} \cdot \mathbf{N}_{\nu}\right) \mathbf{N}_{\nu}^{\perp}
$$

Here the normals $\mathbf{N}_{\nu}$ have been defined after (5.4), in particular their order is fixed. Further, each function $\phi_{\epsilon, \nu}: \mathbb{R} \rightarrow \mathbb{R}$ is piecewise constant, with discontinuity points separated by at least $\epsilon$ and jumps no larger than $n^{\prime} \epsilon$ (see Fig. 6 for an illustration). This implies that

$\varphi_{\epsilon, \nu}^{\mathrm{p}} \in S B V_{\mathrm{loc}}\left(\mathbb{R}^{2} ; \mathbb{R}^{2}\right)$

$\mathcal{J}_{\varphi_{\epsilon, \nu}^{\mathrm{p}}}$ consists of parallel straight infinite lines with normal $\mathbf{N}_{\nu}$ separated at least by distance $\epsilon$,

$D \boldsymbol{\varphi}_{\epsilon, \nu}^{\mathrm{p}}=\mathbf{I} \mathcal{L}^{2}+\mathbf{b}_{\epsilon \nu} \otimes \mathbf{N}_{\nu} \mathcal{H}^{1}\left\lfloor_{\mathcal{J}_{\varphi_{\epsilon, \nu}^{\mathrm{p}}}}, \quad \mathbf{b}_{\epsilon \nu} \cdot \mathbf{N}_{\nu}=0, \quad \mathbf{b}_{\epsilon \nu} \in \mathbb{R}^{2} \quad\right.$ constant on each line, $\left|\mathbf{b}_{\epsilon \nu}\right| \leq n^{\prime} \epsilon$

Let $\omega^{\prime}=\left\{\mathbf{X} \in \omega: \operatorname{dist}(\mathbf{X}, \partial \omega)>L^{2} \epsilon\right\}$. Then the plastic strain $\mathbf{F}_{\epsilon}^{\mathrm{p}}$ is defined by

$$
\mathbf{F}_{\epsilon}^{\mathrm{p}}\left\lfloor_{\omega^{\prime}}=D \varphi_{\epsilon}^{\mathrm{p}}{\left\lfloor\omega^{\prime}\right.}=\mathbf{I} \mathcal{L}^{2}\left\lfloor_{\omega^{\prime}}+\sum_{j} \mathbf{b}_{\epsilon j} \otimes \mathbf{N}_{j} \mathcal{H}^{1}\left\lfloor\mathcal{J}_{j} \cap \omega^{\prime}\right.\right.\right.
$$

Here $\mathcal{J}_{j}$ are the finitely many segments which constitute the jump set of $\varphi_{\epsilon}^{\mathrm{p}}$ in $\omega$, each of which has a constant jump $\mathbf{b}_{\epsilon j}=\llbracket \varphi_{\epsilon}^{\mathrm{p}} \rrbracket$.

Note that we shall show below (Lemma 5.2(ii)) that $D \varphi_{\epsilon}^{\mathrm{p}}$ is uniquely defined in $\omega^{\prime}$ and that this gives a unique definition of $\mathbf{F}_{\epsilon}^{\mathrm{p}}$ from $\boldsymbol{\varphi}_{\epsilon}$ on $\Omega^{\prime} \backslash\left(\bigcup_{k=1}^{N_{\epsilon}} B_{\left(m+L^{2}\right) \epsilon}\left(\mathbf{X}_{k}\right)\right)$, where $\Omega^{\prime}=\{\mathbf{X} \in \Omega: \operatorname{dist}(\mathbf{X}, \partial \Omega)>$ $\left.L^{2} \epsilon\right\}$.

(C3) For any dislocation $\mathbf{X}_{k}$, there is a map $\varphi_{\epsilon}^{\mathrm{e}}$, Lipschitz continuous and one-to-one, such that $\boldsymbol{\varphi}_{\epsilon}$ can be expressed as

$$
\boldsymbol{\varphi}_{\epsilon}(\mathbf{X})=\left(\boldsymbol{\varphi}_{\epsilon}^{\mathrm{e}} \circ \boldsymbol{\varphi}_{\epsilon}^{\mathrm{d}}\right)(\mathbf{X}) \quad \forall \mathbf{X} \in B_{c \epsilon}\left(\mathbf{X}_{k}\right)
$$


The function $\varphi_{\epsilon}^{\mathrm{d}} \in S B V_{\text {loc }}\left(\mathbb{R}^{2} ; \mathbb{R}^{2}\right)$ is explicitly defined from the slip-line normals and the Burgers vectors. Precisely, in each core we are given $N_{d k}$ normals to the jump set $\mathbf{N}_{j}$, cf. (C1), with slips $\mathbf{b}_{\epsilon j} \in \mathbb{R}^{2}$, which obey $\left|\mathbf{b}_{\epsilon j}\right| \leq n^{\prime} \epsilon$ and $\mathbf{b}_{\epsilon j} \cdot \mathbf{N}_{j}=0$ as in (C2). As a matter of convention, $\mathbf{N}_{j}^{\perp}$ will denote the counterclockwise $90^{\circ}$ rotation of $\mathbf{N}_{j}$, and the normals to the jump sets are chosen so that $\mathbf{N}_{j}^{\perp}$ points radially outward from the dislocation center, to the direction of the slip line, see Fig. 7 . Given these parameters, we set

$$
\boldsymbol{\varphi}_{\epsilon}^{\mathrm{d}}(\mathbf{X})=\mathbf{X}-\sum_{j=1}^{N_{d k}} \mathbf{b}_{\epsilon j}^{*}\left(\mathbf{X}-\mathbf{X}_{k}\right) \frac{\theta_{j}\left(\mathbf{X}-\mathbf{X}_{k}\right)}{2 \pi}
$$

where $\mathbf{b}_{\epsilon j}^{*}(\mathbf{X})$ is the smoothed radial slip,

$$
\mathbf{b}_{\epsilon j}^{*}(\mathbf{X})=\frac{\mathbf{X}}{|\mathbf{X}|} \mathbf{N}_{j}^{\perp} \cdot \mathbf{b}_{\epsilon j} \min \left\{\frac{|\mathbf{X}|}{m \epsilon}, 1\right\}
$$

Each function $\mathbf{b}_{\epsilon j}^{*}(\mathbf{X})$ vanishes at the origin, is Lipschitz continuous and radial, in the sense that $\mathbf{b}_{\epsilon j}^{*}(\mathbf{X})$ is parallel to $\mathbf{X}$ for all $\mathbf{X}$. Further, on the part of the slip line outside $B_{m \epsilon}$ it coincides with the "external" slip $\mathbf{b}_{\epsilon j}$, in the sense that

$$
\mathbf{b}_{\epsilon j}^{*}\left(t \mathbf{N}_{j}^{\perp}\right)=\mathbf{b}_{\epsilon j} \quad \text { for all } t \geq m \epsilon .
$$

The angle $\theta_{j}: \mathbb{R}^{2} \rightarrow[-2 \pi, 2 \pi]$ is a function which obeys $\theta_{j}\left(t \mathbf{e}_{1}\right)=0$ for all $t>0$,

$$
\mathbf{X}=|\mathbf{X}|\left(\cos \theta_{j}(\mathbf{X}), \sin \theta_{j}(\mathbf{X})\right) \quad \text { for all } \mathbf{X} \in \mathbb{R}^{2},
$$

and is continuous away from $[0, \infty) \mathbf{N}_{j}^{\perp}$. Clearly $\theta_{j}(t \mathbf{X})=\theta_{j}(\mathbf{X})$ for $t>0$, and $\theta_{j}$ is unique up to the value on the jump.

We remark that $\varphi_{\epsilon}^{\mathrm{d}}$ is a $S B V_{\text {loc }}$ function, with jump set $\mathbf{X}_{k}+\cup_{j}(0, \infty) \mathbf{N}_{j}^{\perp}$. Further, we recall that

$$
m=2 N_{s} n^{\prime},
$$

cf. (C1). This conditions guarantees that $\boldsymbol{\varphi}_{\epsilon}^{\mathrm{d}}$ is bijective. In particular, since all $\mathbf{b}_{\epsilon j}^{*}$ are radial we have $\boldsymbol{\varphi}_{\epsilon}^{\mathrm{d}}\left(\mathbf{X}_{k}+\mathbb{R} \mathbf{v}\right) \subset \mathbf{X}_{k}+\mathbb{R} \mathbf{v}$ for all unit vectors $\mathbf{v}$. An explicit computation shows that, for any unit vector $\mathbf{v}$ and $t \in \mathbb{R}$,

$$
\boldsymbol{\varphi}_{\epsilon}^{\mathrm{d}}\left(\mathbf{X}_{k}+t \mathbf{v}\right)=\mathbf{X}_{k}+\mathbf{v}\left[t-\operatorname{sgn}(t) \min \left\{\frac{|t|}{m \epsilon}, 1\right\} \sum_{j=1}^{N_{d k}} \mathbf{N}_{j}^{\perp} \cdot \mathbf{b}_{\epsilon j} \frac{\theta_{j}(\mathbf{v})}{2 \pi}\right] .
$$

This function is guaranteed to be bijective if $\left(\boldsymbol{\varphi}_{\epsilon}^{\mathrm{d}}-\mathbf{X}_{k}\right) \cdot \mathbf{v}$ is a monotone function of $t$, or equivalently, $\partial\left(\boldsymbol{\varphi}_{\epsilon}^{\mathrm{d}}-\mathbf{X}_{k}\right) \cdot \mathbf{v} / \partial t>0$. Since $\left|\mathbf{b}_{\epsilon j}\right| \leq n^{\prime} \epsilon$, such condition will be satisfied by setting $m$ as in (5.16). An explicit computation shows that

$$
\left|\nabla \varphi_{\epsilon}^{\mathrm{d}}-\mathbf{I}\right| \leq 2 \text {. }
$$

Further, outside the inner core $B_{m \epsilon}\left(\mathbf{X}_{k}\right)$ the jump of $\varphi_{\epsilon}^{\mathrm{d}}$ has the same form as required in (C2), in the sense that

$$
D \boldsymbol{\varphi}_{\epsilon}^{\mathrm{d}} \bigsqcup_{\left(\mathbb{R}^{2} \backslash B_{m \epsilon}\left(\mathbf{X}_{k}\right)\right)}=\nabla \boldsymbol{\varphi}_{\epsilon}^{\mathrm{d}} \mathcal{L}^{2}\left\lfloor_{\left(\mathbb{R}^{2} \backslash B_{m \epsilon}\left(\mathbf{X}_{k}\right)\right)}+\sum_{j=1}^{N_{d k}} \mathbf{b}_{\epsilon j} \otimes \mathbf{N}_{j} \mathcal{H}^{1} \iota_{\left(\mathbf{X}_{k}+(m \epsilon, \infty) \mathbf{N}_{j}^{+}\right)} .\right.
$$

(C4) The total deformation gradient and its elastic and plastic parts are respectively defined as

$$
\begin{aligned}
& \mathbf{F}_{\epsilon}=D \boldsymbol{\varphi}_{\epsilon} \quad \in \mathcal{M}\left(\Omega ; \mathbb{R}^{2 \times 2}\right) \\
& \mathbf{F}_{\epsilon}^{\mathrm{e}}=\nabla \boldsymbol{\varphi}_{\epsilon} \quad \in L^{1}\left(\Omega ; \mathbb{R}^{2 \times 2}\right), \\
& \mathbf{F}_{\epsilon}^{\mathrm{p}}=\mathbf{I} \mathcal{L}^{2}+\sum_{j} \mathbf{b}_{\epsilon j} \otimes \mathbf{N}_{j} \mathcal{H}^{1}\left\lfloor\mathcal{J}_{j} \cap \Omega^{\prime} \quad \in \mathcal{M}\left(\Omega ; \mathbb{R}^{2 \times 2}\right),\right.
\end{aligned}
$$


where $\mathbf{F}_{\epsilon}^{\mathrm{p}}$ is set as $\mathbf{I}$ on $\Omega \backslash \Omega^{\prime}$ and $\Omega^{\prime}$ is defined as above. By (C2) and (C3), the jumpset $\mathcal{J}$ is a union of segments, i.e. $\mathcal{J}=\cup_{j} \mathcal{J}_{j}$, each with a constant vector $\mathbf{b}_{\epsilon j}$. The value of $\mathbf{b}_{\epsilon j}$ inside the core is taken for each $j$ equal to that of the corresponding segment in the compatible region $B_{c \epsilon}\left(\mathbf{X}_{k}\right) \backslash B_{m \epsilon}\left(\mathbf{X}_{k}\right)$. Then Curl $\mathbf{F}_{\epsilon}^{\mathrm{p}}$ in $\Omega^{\prime}$ satisfies, cf. Reina and Conti (2014),

$$
\operatorname{Curl} \mathbf{F}_{\epsilon}^{\mathrm{p}}\left\lfloor_{\Omega^{\prime}}=\sum_{j} \pm \mathbf{b}_{\epsilon j} \mathcal{H}^{0}\left\lfloor_{\left(\partial \mathcal{J}_{j}\right) \cap \Omega^{\prime}}\right.\right.
$$

where the $+\operatorname{sign}$ corresponds to the endpoint of segment $\mathcal{J}_{j}$ pointed by $\mathbf{N}^{\perp}$, and the - sign to the opposite endpoint. The support of Curl $\mathbf{F}_{\epsilon}^{\mathrm{p}}\left\lfloor_{\Omega^{\prime}}\right.$ thus corresponds to the $N_{\epsilon}$ dislocation points.

The fields $\mathbf{F}_{\epsilon}^{\mathrm{e}}$ that result in a finite energy belong to the space $B V\left(\Omega ; \mathbb{R}^{2 \times 2}\right)$ and therefore the traces $\mathbf{F}_{\epsilon}^{\mathrm{e}+}$ and $\mathbf{F}_{\epsilon}^{\mathrm{e}-}$ on each side of the jump set $\mathcal{J}$ are well-defined quantities by Theorem 3.77 of Ambrosio et al. (2000). We then define $\mathbf{F}_{\epsilon}^{\mathrm{e}}$ on the jump set $\mathcal{J}$ as

$$
\mathbf{F}_{\epsilon}^{\mathrm{e}}(\mathbf{X})=\frac{\mathbf{F}_{\epsilon}^{\mathrm{e}+}(\mathbf{X})+\mathbf{F}_{\epsilon}^{\mathrm{e}-}(\mathbf{X})}{2} \quad \forall \mathbf{X} \in \mathcal{J}
$$

although the precise definition will not affect the limiting results.

We first show that $\varphi_{\epsilon}^{\mathrm{p}}$ is approximately Lipschitz.

Lemma 5.1. Let $\varphi_{\epsilon}^{\mathrm{p}}: \mathbb{R}^{2} \rightarrow \mathbb{R}^{2}$ be a pure-slip deformation as defined in (C2), and set $L=2^{N_{s}}\left(1+n^{\prime}\right)^{N_{s}}$. Then the following holds:

(i) $\varphi_{\epsilon}^{\mathrm{p}}$ is invertible, in the sense that there is $\boldsymbol{\varphi}_{\epsilon}^{\mathrm{p},-1}: \mathbb{R}^{2} \rightarrow \mathbb{R}^{2}$ such that $\boldsymbol{\varphi}_{\epsilon}^{\mathrm{p},-1}\left(\boldsymbol{\varphi}_{\epsilon}^{\mathrm{p}}(\mathbf{X})\right)=\boldsymbol{\varphi}_{\epsilon}^{\mathrm{p}}\left(\boldsymbol{\varphi}_{\epsilon}^{\mathrm{p},-1}(\mathbf{X})\right)=$ $\mathbf{X}$ for all $\mathbf{X} \in \mathbb{R}^{2}$.

(ii) For all $\mathbf{X}, \mathbf{Y} \in \mathbb{R}^{2}$ satisfying $|\mathbf{X}-\mathbf{Y}| \geq \epsilon$ one has

$$
\left|\varphi_{\epsilon}^{\mathrm{p}}(\mathbf{X})-\varphi_{\epsilon}^{\mathrm{p}}(\mathbf{Y})\right| \leq L|\mathbf{X}-\mathbf{Y}|
$$

and, similarly, for all $\tilde{\mathbf{X}}, \tilde{\mathbf{Y}} \in \mathbb{R}^{2}$ satisfying $|\tilde{\mathbf{X}}-\tilde{\mathbf{Y}}| \geq \epsilon$,

$$
\left|\varphi_{\epsilon}^{\mathrm{p},-1}(\tilde{\mathbf{X}})-\varphi_{\epsilon}^{\mathrm{p},-1}(\tilde{\mathbf{Y}})\right| \leq L|\tilde{\mathbf{X}}-\tilde{\mathbf{Y}}| .
$$

(iii) For any $\mathbf{X} \in \mathbb{R}^{2}$ and any $\tilde{\mathbf{X}} \in \mathbb{R}^{2}$ one has

$$
\begin{aligned}
& \boldsymbol{\varphi}_{\epsilon}^{\mathrm{p}}\left(B_{\epsilon}(\mathbf{X})\right) \subset B_{L \epsilon}\left(\boldsymbol{\varphi}_{\epsilon}^{\mathrm{p}}(\mathbf{X})\right), \\
& \boldsymbol{\varphi}_{\epsilon}^{\mathrm{p},-1}\left(B_{\epsilon}(\tilde{\mathbf{X}})\right) \subset B_{L \epsilon}\left(\boldsymbol{\varphi}_{\epsilon}^{\mathrm{p},-1}(\tilde{\mathbf{X}})\right) .
\end{aligned}
$$

(iv) Setting $n=\left(1+n^{\prime}\right)^{N_{s}}$ one has, for all $\mathbf{X}$ in the jump set of $\boldsymbol{\varphi}_{\epsilon}^{\mathrm{p}}$,

$$
\left|\llbracket \varphi_{\epsilon}^{\mathrm{p}} \rrbracket\right|(\mathbf{X}) \leq n \epsilon
$$

Here and below we use the overhead tilde to denote quantities in the intermediate (compatible) configuration $\varphi_{\epsilon}^{\mathrm{p}}\left(\mathbb{R}^{2}\right)$.

Proof. Consider a single slip deformation $\varphi_{\epsilon, 1}^{\mathrm{p}}$, cf. (C2). By construction, it can be written as

$$
\varphi_{\epsilon, 1}^{\mathrm{p}}(\mathbf{X})=\mathbf{X}-\phi_{\epsilon}(\mathbf{X} \cdot \mathbf{N}) \mathbf{N}^{\perp}
$$

with $\phi_{\epsilon}: \mathbb{R} \rightarrow \mathbb{R}$ piecewise constant, see Fig. 6. Further, the discontinuity points have a separation of at least $\epsilon$ from each other, and the jumps of $\phi_{\epsilon}$ are no larger than $n^{\prime} \epsilon$. This implies $\left|\phi_{\epsilon}(s)-\phi_{\epsilon}(t)\right| \leq$ $n^{\prime} \epsilon \#\left(\mathcal{J}_{\phi_{\epsilon}} \cap[s, t]\right) \leq n^{\prime}(|t-s|+\epsilon)$ and therefore

$$
\left|\boldsymbol{\varphi}_{\epsilon, 1}^{\mathrm{p}}(\mathbf{X})-\boldsymbol{\varphi}_{\epsilon, 1}^{\mathrm{p}}(\mathbf{Y})\right|+\epsilon \leq\left(1+n^{\prime}\right)(|\mathbf{X}-\mathbf{Y}|+\epsilon) \quad \text { for all } \mathbf{X}, \mathbf{Y} \in \mathbb{R}^{2}
$$




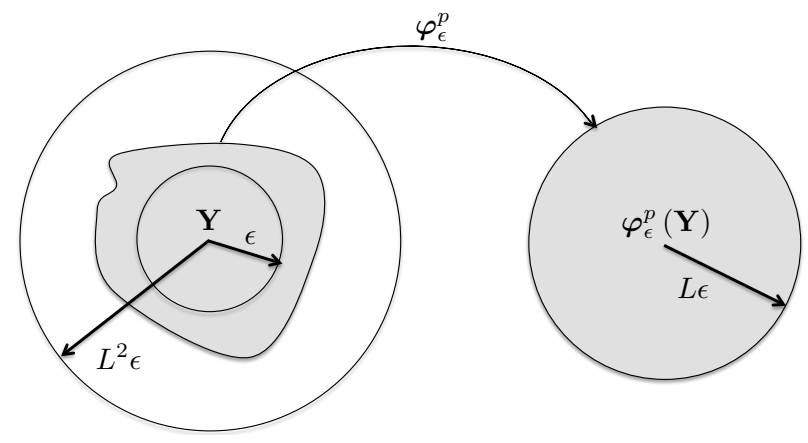

Figure 8: Choice of domains to prove the uniqueness of $\mathbf{F}_{\epsilon}^{\mathrm{p}}$ in $B_{\epsilon}(\mathbf{Y})$, for $B_{L^{2} \epsilon}(\mathbf{Y}) \subset \Omega \backslash \bigcup_{k=1}^{N_{\epsilon} B_{m \epsilon}}\left(\mathbf{X}_{k}\right)$.

The same holds for all $\varphi_{\epsilon, \nu}^{\mathrm{p}}$. Taking the composition we obtain

$$
\left|\boldsymbol{\varphi}_{\epsilon}^{\mathrm{p}}(\mathbf{X})-\varphi_{\epsilon}^{\mathrm{p}}(\mathbf{Y})\right|+\epsilon \leq\left(1+n^{\prime}\right)^{N_{s}}(|\mathbf{X}-\mathbf{Y}|+\epsilon) \quad \text { for all } \mathbf{X}, \mathbf{Y} \in \mathbb{R}^{2} .
$$

Setting $L=2^{N_{s}}\left(1+n^{\prime}\right)^{N_{s}}$ concludes the proof of (5.25). Taking the limit $\mathbf{Y} \rightarrow \mathbf{X}^{+}, \mathbf{X} \rightarrow \mathbf{X}^{-}$proves (5.29).

To prove (i) we observe that (5.30) easily implies that $\varphi_{\epsilon, 1}^{\mathrm{p}}$ is invertible, with inverse

$$
\varphi_{\epsilon, 1}^{\mathrm{p},-1}(\tilde{\mathbf{X}})=\tilde{\mathbf{X}}+\phi_{\epsilon}(\tilde{\mathbf{X}} \cdot \mathbf{N}) \mathbf{N}^{\perp}
$$

where $\phi_{\epsilon}$ is the same function as in (5.30). Then $\varphi_{\epsilon}^{\mathrm{p},-1}$ is defined as the composition of the inverses of the single slips, and the estimate

$$
\left|\varphi_{\epsilon}^{\mathrm{p},-1}(\tilde{\mathbf{X}})-\varphi_{\epsilon}^{\mathrm{p},-1}(\tilde{\mathbf{Y}})\right|+\epsilon \leq\left(1+n^{\prime}\right)^{N_{s}}(|\tilde{\mathbf{X}}-\tilde{\mathbf{Y}}|+\epsilon) \quad \text { for all } \tilde{\mathbf{X}}, \tilde{\mathbf{Y}} \in \mathbb{R}^{2}
$$

follows. This concludes the proof of (i) and (ii). Finally, assertion (iii) follows immediately from (5.32) and (5.34).

Now we prove that $\mathbf{F}_{\epsilon}^{\mathrm{p}}$ is well defined.

Lemma 5.2. Let $\boldsymbol{\varphi}_{\epsilon}, \boldsymbol{\varphi}_{\epsilon}^{\mathrm{e}}, \varphi_{\epsilon}^{\mathrm{p}}$ and $\mathbf{F}_{\epsilon}^{\mathrm{p}}$ as in (C2-C4). Then the following results hold:

(i) The measure $\mathbf{F}_{\epsilon}^{\mathrm{p}}$ is uniquely defined from $\varphi_{\epsilon}$.

(ii) If $\mathbf{Y}$ is such that $\omega=B_{L^{2} \epsilon}(\mathbf{Y}) \subset \Omega \backslash \bigcup_{k=1}^{N_{\epsilon}} B_{m \epsilon}\left(\mathbf{X}_{k}\right)$, and $\boldsymbol{\varphi}_{\epsilon}=\boldsymbol{\varphi}_{\epsilon}^{\mathrm{e}} \circ \boldsymbol{\varphi}_{\epsilon}^{\mathrm{p}}, \boldsymbol{\varphi}_{\epsilon}=\hat{\boldsymbol{\varphi}}_{\epsilon}^{\mathrm{e}} \circ \hat{\boldsymbol{\varphi}}_{\epsilon}^{\mathrm{p}}$ are two decompositions as in (C2) on the domain $\omega$, then on the smaller set $B_{\epsilon}(\mathbf{Y})$ they coincide up to a translation, in the sense that there is $\mathbf{c} \in \mathbb{R}^{2}$ such that $\hat{\boldsymbol{\varphi}}_{\epsilon}^{\mathrm{p}}(\mathbf{X})=\varphi_{\epsilon}^{\mathrm{p}}(\mathbf{X})-\mathbf{c}$ for $\mathbf{X} \in B_{\epsilon}(\mathbf{Y})$ and $\hat{\boldsymbol{\varphi}}_{\epsilon}^{\mathrm{e}}(\tilde{\mathbf{X}})=\varphi_{\epsilon}^{\mathrm{e}}(\tilde{\mathbf{X}}+\mathbf{c})$ for $\tilde{\mathbf{X}} \in \varphi_{\epsilon}^{\mathrm{p}}\left(B_{\epsilon}(\mathbf{Y})\right)$.

Proof. We first show that (ii) implies (i). Recall that $\mathbf{F}_{\epsilon}^{\mathrm{p}}=\mathbf{I}$ outside $\Omega^{\prime}=\left\{\mathbf{X} \in \Omega\right.$ : $\left.\operatorname{dist}(\mathbf{X}, \partial \Omega)>L^{2} \epsilon\right\}$. Every $\mathbf{Y} \in \Omega^{\prime} \backslash \bigcup_{k=1}^{N_{\epsilon}} B_{\left(m+L^{2}\right) \epsilon}\left(\mathbf{X}_{k}\right)$ has the property stated in (ii), therefore $\mathbf{F}_{\epsilon}^{\mathrm{p}}$ is unique on this set. Further, this shows that it is unique in the outer region $B_{c \epsilon}\left(\mathbf{X}_{k}\right) \backslash B_{\left(m+L^{2}\right) \epsilon}\left(\mathbf{X}_{k}\right)$ of each core, and therefore by (C3) also in the interior. This concludes the proof of (i).

It remains to prove (ii). Let $\mathbf{Y}$ be as stated. By the previous Lemma, $\varphi_{\epsilon}^{\mathrm{p}}\left(B_{\epsilon}(\mathbf{Y})\right) \subset B_{L \epsilon}\left(\boldsymbol{\varphi}_{\epsilon}^{\mathrm{p}}(\mathbf{Y})\right)$ and $\varphi_{\epsilon}^{\mathrm{p},-1}\left(B_{L \epsilon}\left(\boldsymbol{\varphi}_{\epsilon}^{\mathrm{p}}(\mathbf{Y})\right)\right) \subset B_{L^{2} \epsilon}(\mathbf{Y})=\omega$, see Fig. 8. We shall show that $D \boldsymbol{\varphi}_{\epsilon}^{\mathrm{p}}=D \hat{\boldsymbol{\varphi}}_{\epsilon}^{\mathrm{p}}$ on $B_{\epsilon}(\mathbf{Y})$ by showing that $\mathcal{J}_{\varphi_{\epsilon}^{\mathrm{p}}}$ and $\mathbf{b}_{\epsilon}$ are uniquely defined from $\varphi_{\epsilon}$ on this set.

Uniqueness of $\mathcal{J}_{\varphi_{\epsilon}^{\mathrm{p}}}$. By definition, $\boldsymbol{\varphi}_{\epsilon}=\boldsymbol{\varphi}_{\epsilon}^{\mathrm{e}} \circ \boldsymbol{\varphi}_{\epsilon}^{\mathrm{p}}$, with $\boldsymbol{\varphi}_{\epsilon}^{\mathrm{p}} \in S B V_{\mathrm{loc}}\left(\mathbb{R}^{2} ; \mathbb{R}^{2}\right)$ and $\boldsymbol{\varphi}_{\epsilon}^{\mathrm{e}}$ Lipschitz continuous. Therefore, by the chain rule, cf. Theorem 3.96 of Ambrosio et al. (2000), and since $\nabla \varphi_{\epsilon}^{\mathrm{p}}=\mathbf{I}$,

$$
D \boldsymbol{\varphi}_{\epsilon}=\left(\tilde{\nabla} \boldsymbol{\varphi}_{\epsilon}^{\mathrm{e}}\right) \circ \boldsymbol{\varphi}_{\epsilon}^{\mathrm{p}} \mathcal{L}^{2}+\left(\boldsymbol{\varphi}_{\epsilon}^{\mathrm{e}}\left(\boldsymbol{\varphi}_{\epsilon}^{\mathrm{p}+}\right)-\boldsymbol{\varphi}_{\epsilon}^{\mathrm{e}}\left(\boldsymbol{\varphi}_{\epsilon}^{\mathrm{p}-}\right)\right) \otimes \mathbf{N} \mathcal{H}^{1}\left\lfloor_{\mathcal{J}_{\varphi_{\epsilon}^{\mathrm{p}}}}\right.
$$


and $\mathcal{J}_{\varphi_{\epsilon}} \subseteq \mathcal{J}_{\varphi_{\epsilon}^{\mathrm{p}}}$. For $\mathbf{X} \in \mathcal{J}_{\varphi_{\epsilon}^{\mathrm{p}}}, \varphi_{\epsilon}^{\mathrm{p}+}(\mathbf{X}) \neq \varphi_{\epsilon}^{\mathrm{p}-}(\mathbf{X})$ and since $\varphi_{\epsilon}^{\mathrm{e}}$ is one-to-one, then $\varphi_{\epsilon}^{\mathrm{e}}\left(\varphi_{\epsilon}^{\mathrm{p}+}(\mathbf{X})\right) \neq$ $\varphi_{\epsilon}^{\mathrm{e}}\left(\boldsymbol{\varphi}_{\epsilon}^{\mathrm{p}-}(\mathbf{X})\right)$. The function $\boldsymbol{\varphi}_{\epsilon}$ is then not approximately continuous in $\mathbf{X}$ and $\mathcal{H}^{1}\left(\mathcal{J}_{\varphi_{\epsilon}^{\mathrm{p}}} \backslash \mathcal{J}_{\boldsymbol{\varphi}_{\epsilon}}\right)=\emptyset$. Therefore, $\mathcal{J}_{\varphi_{\epsilon}^{\mathrm{p}}}=\mathcal{J}_{\varphi_{\epsilon}}$ and $\mathcal{J}_{\varphi_{\epsilon}^{\mathrm{p}}}$ is uniquely defined from $\varphi_{\epsilon}$, up to null sets.

Uniqueness of $\mathbf{b}_{\epsilon}$. We consider two decompositions $\boldsymbol{\varphi}_{\epsilon}=\boldsymbol{\varphi}_{\epsilon}^{\mathrm{e}} \circ \boldsymbol{\varphi}_{\epsilon}^{\mathrm{p}}=\hat{\boldsymbol{\varphi}}_{\epsilon}^{\mathrm{e}} \circ \hat{\boldsymbol{\varphi}}_{\epsilon}^{\mathrm{p}}$ in $B_{L^{2} \epsilon}(\mathbf{Y})$. We write $B_{L^{2} \epsilon}(\mathbf{Y})$ as the union of finitely many non overlapping connected open domains $\mathcal{B}$, i.e. $\omega=\cup \overline{\mathcal{B}}$, such that $\mathcal{B} \cap \mathcal{J}=\emptyset$. For each domain $\mathcal{B}, \varphi_{\epsilon}^{\mathrm{p}}$ and $\hat{\varphi}_{\epsilon}^{\mathrm{p}}$ can be written, by construction, as $\varphi_{\epsilon}^{\mathrm{p}}(\mathbf{X})=\mathbf{X}+\mathbf{c}_{\mathcal{B}}$ and $\hat{\boldsymbol{\varphi}}_{\epsilon}^{\mathrm{p}}(\mathbf{X})=\mathbf{X}+\hat{\mathbf{c}}_{\mathcal{B}}$, with $\mathbf{c}_{\mathcal{B}}$ and $\hat{\mathbf{c}}_{\mathcal{B}}$ constant vectors. Then,

$$
\boldsymbol{\varphi}_{\epsilon}(\mathbf{X})=\left(\boldsymbol{\varphi}_{\epsilon}^{\mathrm{e}} \circ \boldsymbol{\varphi}_{\epsilon}^{\mathrm{p}}\right)(\mathbf{X})=\left(\hat{\boldsymbol{\varphi}}_{\epsilon}^{\mathrm{e}} \circ \hat{\boldsymbol{\varphi}}_{\epsilon}^{\mathrm{p}}\right)(\mathbf{X})=\boldsymbol{\varphi}_{\epsilon}^{\mathrm{e}}\left(\mathbf{X}+\mathbf{c}_{\mathcal{B}}\right)=\hat{\boldsymbol{\varphi}}_{\epsilon}^{\mathrm{e}}\left(\mathbf{X}+\hat{\mathbf{c}}_{\mathcal{B}}\right)
$$

Let $\mathbf{d}_{\mathcal{B}}=\mathbf{c}_{\mathcal{B}}-\hat{\mathbf{c}}_{\mathcal{B}}$. We proceed to show that all blocks $\mathcal{B}$ such that $\mathcal{B} \cap B_{\epsilon}(\mathbf{Y}) \neq \emptyset$ have the same $\mathbf{d}_{\mathcal{B}}$. For that purpose, consider two blocks $\mathcal{B}$ and $\mathcal{B}^{\prime}$ such that they are in contact in the intermediate configuration defined by $\varphi_{\epsilon}^{\mathrm{p}}$, i.e. $\left(\overline{\mathcal{B}}+\mathbf{c}_{\mathcal{B}}\right) \cap\left(\overline{\mathcal{B}}^{\prime}+\mathbf{c}_{\mathcal{B}^{\prime}}\right) \neq \emptyset$. Then, there exists $\mathbf{X} \in \overline{\mathcal{B}}, \mathbf{X}^{\prime} \in \overline{\mathcal{B}}^{\prime}$, such that $\mathbf{X}+\mathbf{c}_{\mathcal{B}}=\mathbf{X}^{\prime}+\mathbf{c}_{\mathcal{B}^{\prime}}$ and $\varphi_{\epsilon}^{\mathrm{e}}\left(\mathrm{X}+\mathbf{c}_{\mathcal{B}}\right)=\varphi_{\epsilon}^{\mathrm{e}}\left(\mathrm{X}^{\prime}+\mathbf{c}_{\mathcal{B}^{\prime}}\right)$. Then, since $\varphi_{\epsilon}=\varphi_{\epsilon}^{\mathrm{e}} \circ \varphi_{\epsilon}^{\mathrm{p}}=\hat{\varphi}_{\epsilon}^{\mathrm{e}} \circ \hat{\varphi}_{\epsilon}^{\mathrm{p}}$ and the elastic deformations are continuous,

$$
\boldsymbol{\varphi}_{\epsilon}^{\mathrm{e}}\left(\mathrm{X}+\mathbf{c}_{\mathcal{B}}\right)=\hat{\varphi}_{\epsilon}^{\mathrm{e}}\left(\mathbf{X}+\hat{\mathbf{c}}_{\mathcal{B}}\right)=\varphi_{\epsilon}^{\mathrm{e}}\left(\mathrm{X}^{\prime}+\mathbf{c}_{\mathcal{B}^{\prime}}\right)=\hat{\varphi}_{\epsilon}^{\mathrm{e}}\left(\mathbf{X}^{\prime}+\hat{\mathbf{c}}_{\mathcal{B}^{\prime}}\right) .
$$

Since $\hat{\varphi}_{\epsilon}^{\mathrm{e}}$ is injective, $\mathbf{X}+\hat{\mathbf{c}}_{\mathcal{B}}=\mathbf{X}^{\prime}+\hat{\mathbf{c}}_{\mathcal{B}^{\prime}}$. Subtracting this equation from $\mathbf{X}+\mathbf{c}_{\mathcal{B}}=\mathbf{X}^{\prime}+\mathbf{c}_{\mathcal{B}^{\prime}}$, one obtains that $\mathbf{d}_{\mathcal{B}}=\mathbf{d}_{\mathcal{B}^{\prime}}$ or, equivalently, that $\mathbf{c}_{\mathcal{B}}-\mathbf{c}_{\mathcal{B}^{\prime}}=\hat{\mathbf{c}}_{\mathcal{B}}-\hat{\mathbf{c}}_{\mathcal{B}^{\prime}}$ for every two pair of blocks in contact in $B_{L \epsilon}\left(\varphi_{\epsilon}^{\mathrm{p}}(\mathbf{Y})\right)$. Since its pullback to the reference configuration contains $B_{\epsilon}(\mathbf{Y})$, one obtains $\mathbf{b}_{\epsilon}=\llbracket \boldsymbol{\varphi}_{\epsilon}^{\mathrm{p}} \rrbracket=\llbracket \hat{\boldsymbol{\varphi}}_{\epsilon}^{\mathrm{p}} \rrbracket=\hat{\mathbf{b}}_{\epsilon}$ on $B_{\epsilon}(\mathbf{Y})$ and the result follows.

The next Lemma shows that the decomposition of $\boldsymbol{\varphi}_{\epsilon}^{\mathrm{p}}$ is unique, and will be used to prove $\operatorname{det} \mathbf{F}^{\mathrm{p}}=1$.

Lemma 5.3. Let $\boldsymbol{\varphi}_{\epsilon} \in X_{\epsilon}$ as defined in (5.4). Consider $\mathbf{Y}$ such that $\operatorname{dist}(\mathbf{Y}, \partial \Omega)>2 L^{2} \epsilon$, with $L$ as in Lemma 5.1 and $B_{L^{2} \epsilon}(\mathbf{Y})$ satisfying $B_{L^{2} \epsilon}(\mathbf{Y}) \cap\left(\bigcup_{k=1}^{N_{\epsilon}} B_{m \epsilon}\left(\mathbf{X}_{k}\right)\right)=\emptyset$. If $\boldsymbol{\varphi}_{\epsilon}^{\mathrm{p}}$ and $\hat{\boldsymbol{\varphi}}_{\epsilon}^{\mathrm{p}}$ are two deformations on $B_{L^{2} \epsilon}(\mathbf{Y})$, satisfying $\mathbf{F}_{\epsilon}^{\mathrm{p}}=D \boldsymbol{\varphi}_{\epsilon}^{\mathrm{p}}=D \hat{\boldsymbol{\varphi}}_{\epsilon}^{\mathrm{p}}$, each with its decomposition of the form

$$
\begin{aligned}
& \boldsymbol{\varphi}_{\epsilon}^{\mathrm{p}}=\varphi_{\epsilon, N_{s}}^{\mathrm{p}} \circ \ldots \circ \varphi_{\epsilon, \nu}^{\mathrm{p}} \circ \ldots \circ \varphi_{\epsilon, 1}^{\mathrm{p}}, \\
& \hat{\varphi}_{\epsilon}^{\mathrm{p}}=\hat{\varphi}_{\epsilon, N_{s}}^{\mathrm{p}} \circ \ldots \circ \hat{\varphi}_{\epsilon, \nu}^{\mathrm{p}} \circ \ldots \circ \hat{\varphi}_{\epsilon, 1}^{\mathrm{p}},
\end{aligned}
$$

then there are constant vectors $\mathbf{c}_{\nu}, \nu=0, . ., N_{s}$ such that

$$
\begin{aligned}
& \boldsymbol{\varphi}_{\epsilon, \nu}^{\mathrm{p}}(\mathbf{X})=\hat{\boldsymbol{\varphi}}_{\epsilon, \nu}^{\mathrm{p}}\left(\mathbf{X}-\mathbf{c}_{\nu-1}\right)+\mathbf{c}_{\nu}, \\
& D \boldsymbol{\varphi}_{\epsilon, \nu}^{\mathrm{p}}\left(\varphi_{\epsilon, \nu-1}^{\mathrm{p}} \circ \ldots \circ \varphi_{\epsilon, 1}^{\mathrm{p}}\right)=D \hat{\varphi}_{\epsilon, \nu}^{\mathrm{p}}\left(\hat{\varphi}_{\epsilon, \nu-1}^{\mathrm{p}} \circ \ldots \circ \hat{\varphi}_{\epsilon, 1}^{\mathrm{p}}\right)
\end{aligned}
$$

in $B_{\epsilon}(\mathbf{Y})$.

Proof. Consider two possible plastic deformation mappings $\varphi_{\epsilon}^{\mathrm{p}}, \hat{\varphi}_{\epsilon}^{\mathrm{p}}$ on $B_{L^{2} \epsilon}(\mathbf{Y})$ and their decomposition as in (5.38). By Lemma 5.2, $\mathbf{F}_{\epsilon}^{\mathrm{p}}=D \boldsymbol{\varphi}_{\epsilon}^{\mathrm{p}}=D \hat{\boldsymbol{\varphi}}_{\epsilon}^{\mathrm{p}}$ on $B_{L^{2} \epsilon}(\mathbf{Y})$, and by integration one obtains $\boldsymbol{\varphi}_{\epsilon}^{\mathrm{p}}=\hat{\boldsymbol{\varphi}}_{\epsilon}^{\mathrm{p}}+\mathbf{c}_{N_{s}}$, where $\mathbf{c}_{N_{s}}$ corresponds to a rigid translation. Define $\bar{\varphi}_{\epsilon, N_{s}}^{\mathrm{p}}(\mathbf{X})=\hat{\varphi}_{\epsilon, N_{s}}^{\mathrm{p}}(\mathbf{X})+\mathbf{c}_{N_{s}}$. Then,

$$
\boldsymbol{\varphi}_{\epsilon, N_{s}}^{\mathrm{p}} \circ \boldsymbol{\varphi}_{\epsilon, N_{s}-1}^{\mathrm{p}} \circ \ldots \circ \varphi_{\epsilon, 1}^{\mathrm{p}}=\bar{\varphi}_{\epsilon, N_{s}}^{\mathrm{p}} \circ \hat{\varphi}_{\epsilon, N_{s}-1}^{\mathrm{p}} \circ \ldots \circ \hat{\boldsymbol{\varphi}}_{\epsilon, 1}^{\mathrm{p}} \quad \text { on } B_{L^{2} \epsilon}(\mathbf{Y}) .
$$

The last deformation applied in the sequence corresponds to slip along jump sets with normal $\mathbf{N}_{N_{s}}$. Since the normals $\mathbf{N}_{\nu}, \nu=1, \ldots, N_{s}$ are all distinct, the jump set with normal $\mathbf{N}_{N_{s}}$ on configuration $\boldsymbol{\varphi}_{\epsilon, N_{s}-1}^{\mathrm{p}} \circ \ldots \circ \boldsymbol{\varphi}_{\epsilon, 1}^{\mathrm{p}}\left(B_{L^{2} \epsilon}(\mathbf{Y})\right)$ will necessarily traverse regions where $\mathbf{F}_{\epsilon}^{\mathrm{p}}=\mathbf{I}$. The segments in those regions will suffer a translation upon pullback to the reference configuration, and will thus have that same normal $\mathbf{N}_{N_{s}}$, each with a given Burgers vector. The ensemble of segments with normal $\mathbf{N}_{N_{s}}$ will, by construction, form a set of straight lines in the plastically deformed configuration with a constant Burgers vector per line, potentially different between lines. Thus, $D \boldsymbol{\varphi}_{\epsilon, N_{s}}^{\mathrm{p}}$ is uniquely characterized from $\mathbf{F}_{\epsilon}^{\mathrm{p}}$ on the set $\boldsymbol{\varphi}_{\epsilon}^{\mathrm{p}}\left(B_{L^{2} \epsilon}(\mathbf{Y})\right)$, which implies $D \varphi_{\epsilon, N_{s}}^{\mathrm{p},-1}=D \bar{\varphi}_{\epsilon, N_{s}}^{\mathrm{p},-1}$ and

$$
\begin{aligned}
& \boldsymbol{\varphi}_{\epsilon, N_{s}}^{\mathrm{p},-1}(\tilde{\mathbf{X}})=\bar{\varphi}_{\epsilon, N_{s}}^{\mathrm{p},-1}(\tilde{\mathbf{X}})+\mathbf{c}_{N_{s}-1}=\hat{\varphi}_{\epsilon, N_{s}}^{\mathrm{p},-1}\left(\tilde{\mathbf{X}}-\mathbf{c}_{N_{s}}\right)+\mathbf{c}_{N_{s}-1} \quad \text { on } D_{N_{s}}, \\
& \varphi_{\epsilon, N_{s}}^{\mathrm{p}}(\mathbf{X})=\bar{\varphi}_{\epsilon, N_{s}}^{\mathrm{p}}\left(\mathbf{X}-\mathbf{c}_{N_{s}-1}\right)=\hat{\varphi}_{\epsilon, N_{s}}^{\mathrm{p}}\left(\mathbf{X}-\mathbf{c}_{N_{s}-1}\right)+\mathbf{c}_{N_{s}} \quad \text { on } \boldsymbol{\varphi}_{\epsilon, N_{s}}^{\mathrm{p},-1}\left(D_{N_{s}}\right),
\end{aligned}
$$


where $D_{N_{s}}=B_{L \epsilon}\left(\varphi_{\epsilon}^{\mathrm{p}}(\mathbf{Y})\right) \subset \varphi_{\epsilon}^{\mathrm{p}}\left(B_{L^{2} \epsilon}(\mathbf{Y})\right)$ and $\mathbf{c}_{N_{s}-1}$ and $\mathbf{c}_{N_{s}}$ are constant vectors. We define $E_{N_{s}}=$ $\varphi_{\epsilon}^{\mathrm{p},-1}\left(D_{N_{s}}\right)$ so that

$$
\boldsymbol{\varphi}_{\epsilon, N_{s}-1}^{\mathrm{p}} \circ \ldots \circ \boldsymbol{\varphi}_{\epsilon, 1}^{\mathrm{p}}=\hat{\boldsymbol{\varphi}}_{\epsilon, N_{s}-1}^{\mathrm{p}} \circ \ldots \circ \hat{\boldsymbol{\varphi}}_{\epsilon, 1}^{\mathrm{p}}+\mathbf{c}_{N_{s}-1} \quad \text { on } E_{N_{s}} .
$$

Defining $\bar{\varphi}_{\epsilon, N_{s}-1}^{\mathrm{p}}(\mathbf{X})=\hat{\boldsymbol{\varphi}}_{\epsilon, N_{s}-1}^{\mathrm{p}}(\mathbf{X})+\mathbf{c}_{N_{s}-1}$, it is readily obtained

$$
\boldsymbol{\varphi}_{\epsilon, N_{s}-1}^{\mathrm{p}} \circ \boldsymbol{\varphi}_{\epsilon, N_{s}-2}^{\mathrm{p}} \circ \ldots \circ \varphi_{\epsilon, 1}^{\mathrm{p}}=\overline{\boldsymbol{\varphi}}_{\epsilon, N_{s}-1}^{\mathrm{p}} \circ \hat{\varphi}_{\epsilon, N_{s}-2}^{\mathrm{p}} \circ \ldots \circ \hat{\boldsymbol{\varphi}}_{\epsilon, 1}^{\mathrm{p}} \quad \text { on } E_{N_{s}} .
$$

The above procedure may be iterated for $\nu=N_{s}-1$ successively till $\nu=1$, obtaining for all $\nu$

$$
\begin{aligned}
& \bar{\varphi}_{\epsilon, \nu}^{\mathrm{p}}(\mathbf{X})=\hat{\varphi}_{\epsilon, \nu}^{\mathrm{p}}(\mathbf{X})+\mathbf{c}_{\nu}, \\
& \boldsymbol{\varphi}_{\epsilon, \nu}^{\mathrm{p}}(\mathbf{X})=\overline{\boldsymbol{\varphi}}_{\epsilon, \nu}^{\mathrm{p}}\left(\mathbf{X}-\mathbf{c}_{\nu-1}\right)=\hat{\boldsymbol{\varphi}}_{\epsilon, \nu}^{\mathrm{p}}\left(\mathbf{X}-\mathbf{c}_{\nu-1}\right)+\mathbf{c}_{\nu} \quad \text { on } \boldsymbol{\varphi}_{\epsilon, \nu}^{\mathrm{p},-1}\left(D_{\nu}\right), \\
& D \boldsymbol{\varphi}_{\epsilon, \nu}^{\mathrm{p}}(\mathbf{X})=D \bar{\varphi}_{\epsilon, \nu}^{\mathrm{p}}\left(\mathbf{X}-\mathbf{c}_{\nu-1}\right)=D \hat{\varphi}_{\epsilon, \nu}^{\mathrm{p}}\left(\mathbf{X}-\mathbf{c}_{\nu-1}\right) \quad \text { on } \boldsymbol{\varphi}_{\epsilon, \nu}^{\mathrm{p},-1}\left(D_{\nu}\right), \\
& \boldsymbol{\varphi}_{\epsilon, \nu-1}^{\mathrm{p}} \circ \boldsymbol{\varphi}_{\epsilon, \nu-2}^{\mathrm{p}} \circ \ldots \circ \boldsymbol{\varphi}_{\epsilon, 1}^{\mathrm{p}}=\bar{\varphi}_{\epsilon, \nu-1}^{\mathrm{p}} \circ \hat{\boldsymbol{\varphi}}_{\epsilon, \nu-2}^{\mathrm{p}} \circ \ldots \circ \hat{\boldsymbol{\varphi}}_{\epsilon, 1}^{\mathrm{p}}, \quad \text { on } E_{\nu},
\end{aligned}
$$

where $D_{\nu}=B_{L \epsilon /\left(2+2 n^{\prime}\right)^{N_{s}-\nu}}\left(\left(\varphi_{\epsilon, \nu}^{\mathrm{p}} \circ \ldots \circ \varphi_{\epsilon, 1}^{\mathrm{p}}\right)(\mathbf{Y})\right), E_{\nu}=\left(\varphi_{\epsilon, \nu}^{\mathrm{p}} \circ . . \circ \varphi_{\epsilon, 1}^{\mathrm{p}}\right)^{-1}\left(D_{\nu}\right)$ and $\mathbf{c}_{\nu}$ are constant vectors. By (5.31), $D_{\nu} \subset \varphi_{\nu+1}^{\mathrm{p},-1}\left(D_{\nu+1}\right)$. Note that for $\nu=2$, the last expression gives $\varphi_{\epsilon, 1}^{\mathrm{p}}(\mathbf{X})=\bar{\varphi}_{\epsilon, 1}^{\mathrm{p}}(\mathbf{X})$, and therefore $\mathbf{c}_{0}=0$.

The composition of two consecutive deformation mappings then gives

$$
\boldsymbol{\varphi}_{\epsilon, \nu}^{\mathrm{p}}\left(\boldsymbol{\varphi}_{\epsilon, \nu-1}^{\mathrm{p}}(\mathbf{X})\right)=\hat{\boldsymbol{\varphi}}_{\epsilon, \nu}^{\mathrm{p}}\left(\varphi_{\epsilon, \nu-1}^{\mathrm{p}}(\mathbf{X})-\mathbf{c}_{\nu-1}\right)+\mathbf{c}_{\nu}=\hat{\boldsymbol{\varphi}}_{\epsilon, \nu}^{\mathrm{p}}\left(\hat{\boldsymbol{\varphi}}_{\epsilon, \nu-1}^{\mathrm{p}}\left(\mathbf{X}-\mathbf{c}_{\nu-2}\right)\right)+\mathbf{c}_{\nu}
$$

and the composition of the first $\nu$ mappings yields

$$
\boldsymbol{\varphi}_{\epsilon, \nu}^{\mathrm{p}} \circ \boldsymbol{\varphi}_{\epsilon, \nu-1}^{\mathrm{p}} \circ \ldots \circ \boldsymbol{\varphi}_{\epsilon, 1}^{\mathrm{p}}=\hat{\boldsymbol{\varphi}}_{\epsilon, \nu}^{\mathrm{p}} \circ \hat{\boldsymbol{\varphi}}_{\epsilon, \nu-1}^{\mathrm{p}} \circ \ldots \circ \hat{\boldsymbol{\varphi}}_{\epsilon, 1}^{\mathrm{p}}\left(\mathbf{X}-\mathbf{c}_{0}\right)+\mathbf{c}_{\nu} \quad \text { on } E_{1},
$$

where $\mathbf{c}_{0}=0$. Thus, from (5.47),

$$
D \boldsymbol{\varphi}_{\epsilon, \nu}^{\mathrm{p}}\left(\boldsymbol{\varphi}_{\epsilon, \nu-1}^{\mathrm{p}} \circ \ldots \circ \boldsymbol{\varphi}_{\epsilon, 1}^{\mathrm{p}}\right)=D \hat{\boldsymbol{\varphi}}_{\epsilon, \nu}^{\mathrm{p}}\left(\boldsymbol{\varphi}_{\epsilon, \nu-1}^{\mathrm{p}} \circ \ldots \circ \boldsymbol{\varphi}_{\epsilon, 1}^{\mathrm{p}}-\mathbf{c}_{\nu-1}\right)=D \hat{\boldsymbol{\varphi}}_{\epsilon, \nu}^{\mathrm{p}}\left(\hat{\boldsymbol{\varphi}}_{\epsilon, \nu-1}^{\mathrm{p}} \circ \ldots \circ \hat{\boldsymbol{\varphi}}_{\epsilon, 1}^{\mathrm{p}}\right) \quad \text { on } E_{1} .
$$

We note that $B_{\epsilon}(\mathbf{Y}) \subset E_{1}$, and $E_{\nu} \subset E_{\nu+1}$, and thus Eqs. (5.39) hold for all $\nu$.

Lemma 5.4. Let $\mathcal{J}$ be the jump set of $\varphi_{\epsilon} \in X_{\epsilon}$. Then, the following results hold:

(i) There exists $A>0$ such that for any ball $B_{r}(\mathbf{X}) \subset \mathbb{R}^{2}$ of radius $r>\epsilon$

$$
\left|\mathcal{J} \cap B_{r}(\mathbf{X})\right| \leq \frac{A r^{2}}{\epsilon} .
$$

(ii) There exists $C^{*}>0$ depending only on $\Omega$ such that

$$
|\mathcal{J} \cap \Omega| \leq \frac{C^{*}}{\epsilon} .
$$

We recall that by definition $\mathcal{J} \subset \Omega$.

Proof. Consider first a ball $B_{\epsilon}(\mathbf{Y})$ such that $B_{2 L \epsilon}(\mathbf{Y}) \cap\left(\bigcup_{k=1}^{N_{\epsilon}} B_{m \epsilon}\left(\mathbf{X}_{k}\right)\right)=\emptyset$. In this ball, the representation of $\varphi_{\epsilon}^{\mathrm{p}}$ from (C2) holds. Since the $\varphi_{\epsilon, \nu}^{\mathrm{p}}$ are piecewise translations and the normals $N_{\nu}$ are all different, the jump set of $\varphi_{\epsilon}^{\mathrm{p}}$ is the union of the counterimages of the jump sets of the individual $\varphi_{\epsilon, \nu}^{\mathrm{p}}$,

$$
\mathcal{J}_{\boldsymbol{\varphi}_{\epsilon}^{\mathrm{p}}}=\mathcal{J}_{\boldsymbol{\varphi}_{\epsilon, 1}^{\mathrm{p}}} \cup \boldsymbol{\varphi}_{\epsilon, 1}^{\mathrm{p},-1}\left(\mathcal{J}_{\boldsymbol{\varphi}_{\epsilon, 2}^{\mathrm{p}}}\right) \cup \cdots \cup\left(\boldsymbol{\varphi}_{\epsilon, 1}^{\mathrm{p},-1} \circ \cdots \circ \varphi_{\epsilon, N_{s}-1}^{\mathrm{p},-1}\right)\left(\mathcal{J}_{\boldsymbol{\varphi}_{\epsilon, N_{s}}^{\mathrm{p}}}\right) .
$$

For every $\nu$, let $\mathbf{Y}_{\nu}=\left(\varphi_{\epsilon, \nu-1}^{\mathrm{p}} \circ \cdots \circ \varphi_{\epsilon, 1}^{\mathrm{p}}\right)(\mathbf{Y})$. Since the jump set of $\varphi_{\epsilon, \nu}^{\mathrm{p}}$ consists of parallel lines with distance at least $\epsilon$, we have

$$
\left|\mathcal{J}_{\varphi_{\epsilon, \nu}^{\mathrm{p}}} \cap B_{L \epsilon}\left(\mathbf{Y}_{\nu}\right)\right| \leq(2 L+1) 2 L \epsilon
$$


and, recalling that $\left(\varphi_{\epsilon, \nu-1}^{\mathrm{p}} \circ \cdots \circ \varphi_{\epsilon, 1}^{\mathrm{p}}\right)\left(B_{\epsilon}(\mathbf{Y})\right) \subset B_{L \epsilon}\left(\mathbf{Y}_{\nu}\right)$, cf. Lemma 5.1(iii),

$$
\left|\left(\boldsymbol{\varphi}_{\epsilon, 1}^{\mathrm{p},-1} \circ \cdots \circ \boldsymbol{\varphi}_{\epsilon, \nu-1}^{\mathrm{p},-1}\right)\left(\mathcal{J}_{\boldsymbol{\varphi}_{\epsilon, \nu}^{\mathrm{p}}}\right) \cap B_{\epsilon}(\mathbf{Y})\right| \leq(2 L+1) 2 L \epsilon .
$$

Therefore $\left|\mathcal{J}_{\boldsymbol{\varphi}_{\epsilon}^{\mathrm{p}}} \cap B_{\epsilon}(\mathbf{Y})\right| \leq N_{s}(2 L+1) 2 L \epsilon$.

Consider now a generic ball $B_{r}(\mathbf{X})$. We cover $B_{r}(\mathbf{X}) \backslash\left(\bigcup_{k=1}^{N_{\epsilon}} B_{c \epsilon}\left(\mathbf{X}_{k}\right)\right)$ with balls $\omega_{l}=B_{\epsilon}\left(\mathbf{Y}_{l}\right)$ with finite overlap such that $B_{2 L \epsilon}\left(\mathbf{Y}_{l}\right) \cap\left(\bigcup_{k=1}^{N_{\epsilon}} B_{m \epsilon}\left(\mathbf{X}_{k}\right)\right)=\emptyset$. The number of those sets $\omega_{l}$ is bounded by $A_{1} r^{2} / \epsilon^{2}$ for some constant $A_{1}$. Similarly, by (5.6) the number of cores intersecting $B_{r}(\mathbf{X})$ is bounded by $A_{2} r^{2} / \epsilon^{2}$ for some constant $A_{2}$, and the total length of the jump set in a core is not larger than $c \epsilon N_{s}$. Then,

$$
\begin{aligned}
\left|\mathcal{J} \cap B_{r}\right| & \leq \sum_{l}\left|\mathcal{J} \cap \omega_{l}\right|+\sum_{k=1}^{N_{\epsilon}}\left|\mathcal{J} \cap\left(B_{r}(\mathbf{X}) \cap B_{c \epsilon}\left(\mathbf{X}_{k}\right)\right)\right| \\
& \leq A_{1} \frac{r^{2}}{\epsilon^{2}} N_{s}(2 L+1) 2 L \epsilon+A_{2} \frac{r^{2}}{\epsilon^{2}} N_{s} c \epsilon \leq A \frac{r^{2}}{\epsilon}
\end{aligned}
$$

which proves (i).

For assertion (ii), since $\Omega$ is a bounded domain, it suffices to cover it with balls of the two types used in (i), or to choose in (i) a ball which covers $\Omega$.

The next Lemma will be used to show that the elastic energy associated to cores vanishes in the continuum limit $\epsilon \rightarrow 0$.

Lemma 5.5. Let $\boldsymbol{\varphi}_{\epsilon}^{\mathrm{d}}$ be as defined in (5.12), and $N_{\epsilon}$ and $B_{c \epsilon}\left(\mathbf{X}_{k}\right)$ as defined in (C1) and (C3). Then there exists $C_{c}>0$ such that

$$
\sum_{k=1}^{N_{\epsilon}} \int_{B_{c \epsilon}\left(\mathbf{X}_{k}\right)}\left|\nabla \varphi_{\epsilon}^{\mathrm{d}}\right|^{2} d X \leq C_{c} \epsilon
$$

Proof. From (5.18) and scaling (5.5), the result is readily obtained

$$
\sum_{k=1}^{N_{\epsilon}} \int_{B_{c \epsilon}\left(\mathbf{X}_{k}\right)}\left|\nabla \boldsymbol{\varphi}_{\epsilon}^{\mathrm{d}}\right|^{2} d X \leq 16 \sum_{k=1}^{N_{\epsilon}}\left|B_{c \epsilon}\left(\mathbf{X}_{k}\right)\right| \leq C_{c} \epsilon
$$

In closing this Section we show that the decomposition we assumed for the plastic strain is generically admissible. This is not used in the current argument, but illustrates the generality of our assumptions.

Lemma 5.6. Consider an arbitrary $\mathbf{F}^{\mathrm{p}} \in C^{\infty}\left(\Omega ; \mathbb{R}^{2 \times 2}\right)$ such that $\operatorname{det} \mathbf{F}^{\mathrm{p}}=1, F_{11}^{\mathrm{p}} \neq 0$ and $F_{12}^{\mathrm{p}} \neq F_{11}^{\mathrm{p}}$. Then $\mathbf{F}^{\mathrm{p}}$ admits a unique description as the composition of the following three sequential simple shear deformations

$$
\mathbf{F}^{\mathrm{p}}=\left(\mathbf{I}-\gamma \mathbf{e}_{2} \otimes \mathbf{e}_{1}\right)\left(\mathbf{I}+\eta \mathbf{e}_{1} \otimes \mathbf{e}_{2}\right)\left(\mathbf{I}+\mu \mathbf{e}_{3}^{\perp} \otimes \mathbf{e}_{3}\right)
$$

with $\mathbf{e}_{3}=\sqrt{2} / 2\left(\mathbf{e}_{1}+\mathbf{e}_{2}\right)$. In particular,

$$
\begin{aligned}
& \mu=2 \frac{F_{11}^{\mathrm{p}}-1}{F_{12}^{\mathrm{p}}-F_{11}^{\mathrm{p}}} \\
& \eta=1+F_{12}^{\mathrm{p}}-F_{11}^{\mathrm{p}} \\
& \gamma=\frac{1+F_{21}^{\mathrm{p}}-F_{22}^{\mathrm{p}}}{F_{12}^{\mathrm{p}}-F_{11}^{\mathrm{p}}},
\end{aligned}
$$

where $F_{11}^{\mathrm{p}}, F_{12}^{\mathrm{p}}, F_{21}^{\mathrm{p}}$ and $F_{22}^{\mathrm{p}}$ are the components of $\mathbf{F}^{\mathrm{p}}$. 
Proof. Expanding the product in (5.57) one obtains

$$
\mathbf{F}^{\mathrm{p}}=\left(\begin{array}{cc}
F_{11}^{\mathrm{p}} & F_{12}^{\mathrm{p}} \\
F_{21}^{\mathrm{p}} & F_{22}^{\mathrm{p}}
\end{array}\right)=\left(\begin{array}{cc}
1-\frac{\mu}{2}+\eta \frac{\mu}{2} & -\frac{\mu}{2}+\eta+\eta \frac{\mu}{2} \\
-\gamma+\gamma \frac{\mu}{2}-\frac{\mu}{2} \gamma \eta+\frac{\mu}{2} & \gamma \frac{\mu}{2}-\gamma \eta-\gamma \eta \frac{\mu}{2}+1+\frac{\mu}{2}
\end{array}\right) .
$$

From the expressions of $F_{11}^{\mathrm{p}}=1+\frac{\mu}{2}(\eta-1)$ and $F_{12}^{\mathrm{p}}=\eta+\frac{\mu}{2}(\eta-1)$, one obtains $\eta=1+F_{12}^{\mathrm{p}}-F_{11}^{\mathrm{p}}$. If $F_{12}^{\mathrm{p}} \neq F_{11}^{\mathrm{p}}$, then, plugging the result into the equation for $F_{11}^{\mathrm{p}}$ we get $F_{11}^{\mathrm{p}}=1+\frac{\mu}{2}\left(F_{12}^{\mathrm{p}}-F_{11}^{\mathrm{p}}\right)$, and so $(5.58)$. Finally, the expression for $F_{21}^{\mathrm{p}}$ simplifies to

$$
F_{21}^{\mathrm{p}}=\frac{\mu}{2}+\gamma\left[-\frac{\mu}{2}(\eta-1)-1\right]=\frac{\mu}{2}-\gamma F_{11}^{\mathrm{p}}
$$

Using the previous result for $\mu$, and the fact that $\operatorname{det} \mathbf{F}^{\mathrm{p}}=1$, one obtains

$$
\gamma F_{11}^{\mathrm{p}}=\frac{F_{11}^{\mathrm{p}}-F_{11}^{\mathrm{p}} F_{22}^{\mathrm{p}}+F_{11}^{\mathrm{p}} F_{21}^{\mathrm{p}}}{F_{12}^{\mathrm{p}}-F_{11}^{\mathrm{p}}}
$$

which simplifies to $(5.60)$ if $F_{11}^{\mathrm{p}} \neq 0$.

We note that the choice of the orientation of the three slip systems was arbitrary and different orientations may be chosen. The conditions for obtaining a unique representation under such choice will then differ from that of this lemma.

\section{Main results.}

We first show that the multiplicative decomposition approximately holds at the mesoscopic level, i.e. $D \varphi_{\epsilon} \sim$ $\mathbf{F}_{\epsilon}^{\mathrm{e}} \mathbf{F}_{\epsilon}^{\mathrm{p}}$. This is one key estimate for our result, and gives a bound both on the regular part (which will constitute $\mathbf{F}^{\mathrm{e}}$ ) and on the jump part. Indeed, both $D \boldsymbol{\varphi}_{\epsilon}$ and $\mathbf{F}_{\epsilon}^{\mathrm{p}}$ are measures, containing singular contributions on the slip lines.

Proposition 6.1. Let $\boldsymbol{\varphi}_{\epsilon} \in X_{\epsilon}$ as defined in (5.4), $\mathbf{F}_{\epsilon}^{\mathrm{e}}$ and $\mathbf{F}_{\epsilon}^{\mathrm{p}}$ as defined in (5.21) and (5.22) respectively and $L$ as defined in Lemma 5.1. Assume $\sup _{\epsilon} E_{\epsilon}\left(\varphi_{\epsilon}\right)<\infty$. Then, there is $C_{1}$ depending on $\sup _{\epsilon} E_{\epsilon}\left(\varphi_{\epsilon}\right)$, such that for any set $K$ compactly contained in $\Omega$ and for any $\epsilon \in(0,1)$ satisfying $\epsilon<\operatorname{dist}(\partial \Omega, K) /\left(2 L^{2}\right)$

$$
\left|D \varphi_{\epsilon}-\mathbf{F}_{\epsilon}^{\mathrm{e}} \mathbf{F}_{\epsilon}^{\mathrm{p}}\right|(K) \leq C_{1} \epsilon^{1 / 2}
$$

Proof. We decompose the proof into three steps (S1)-(S3) that concern, respectively, the dislocation cores, the compatible subdomains away from the dislocations and, finally, the total domain. These intermediate results will often use a combination of the Boundary Trace theorem (Theorem 3.87 of Ambrosio et al. (2000)) and the Poincaré inequality (Theorem 3.44 of Ambrosio et al. (2000)) for $B V$ functions, that we make precise here. Note that the constant in the Boundary Trace theorem depends on the domain. If the domain is a ball $B_{1}$ of radius 1 , the theorem asserts $\int_{\partial B_{1}}|f| d \mathcal{H}^{1} \leq C_{t 1}\|f\|_{B V\left(B_{1}\right)}=C_{t 1} \int_{B_{1}}|f| d X+C_{t 1}|D f|\left(B_{1}\right)$ for some constant $C_{t 1}>0$. If $B_{1}$ is replaced by $B_{\epsilon}$, where $B_{\epsilon}$ represents a ball of radius $\epsilon$, a scaling argument yields the inequality $\int_{\partial B_{\epsilon}}|f| d \mathcal{H}^{1} \leq \frac{C_{t 1}}{\epsilon} \int_{B_{\epsilon}}|f| d X+C_{t 1}|D f|\left(B_{\epsilon}\right)$ for the same constant $C_{t 1}$ as before. In particular, if $f \in B V\left(B_{\epsilon}\right)$, then

$$
\int_{\partial B_{\epsilon}}|f-\bar{f}| d \mathcal{H}^{1} \leq C_{t 1}|D f|\left(B_{\epsilon}\right)+\frac{C_{t_{1}}}{\epsilon} \int_{B_{\epsilon}}|f-\bar{f}| d X \leq C_{t p}|D f|\left(B_{\epsilon}\right),
$$

where $\bar{f}=\left|B_{\epsilon}\right|^{-1} \int_{B_{\epsilon}} f d X$ and the second inequality follows from Poincaré's inequality, see also Ambrosio et al. (2000) [Remark 3.45] for a corresponding scaling argument. The constants $C_{t 1}$ and $C_{t p}$ are independent of $\epsilon$ and of course, independent of $f$. The above result is also true for $\mathcal{J} \cap B_{\epsilon}$,

$$
\int_{\mathcal{J} \cap B_{\epsilon}}|f-\bar{f}| d \mathcal{H}^{1} \leq C_{t p^{\prime}}|D f|\left(B_{\epsilon}\right)
$$




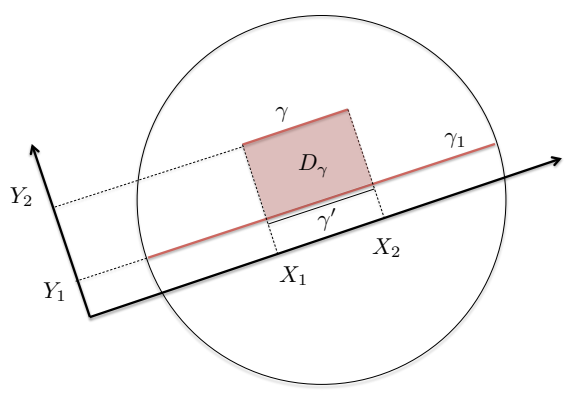

Figure 9: Sketch of the strategy to bound the absolute value of a function over a segment in a ball, with the variation of the function over the ball.

as long as $\mathcal{J} \cap B_{\epsilon}$ is the union of at most $M$ segments, where the constant $C_{t p^{\prime}}$ depends only on $M$. This estimate holds for $f$ on the jump set being defined as either one of the two one-sided traces $f^{+}$and $f^{-}$, or as the average $\left(f^{+}+f^{-}\right) / 2$. To see this, let $C_{p 1}$ be the constant entering the estimate corresponding to (6.2) for the half ball, which does not depend on the orientation of the half ball. Given a segment $\gamma \subset B_{\epsilon}$, consider the diameter $\gamma_{1}$ parallel to $\gamma$, and let $\gamma^{\prime}$ be the projection of $\gamma$ onto $\gamma_{1}$. By the reverse triangle inequality and the fundamental theorem of calculus, working for notational simplicity in coordinates such that $\gamma$ is parallel to $e_{1}$,

$$
\begin{aligned}
& \left|\int_{\gamma}\right| f-\bar{f}\left|d \mathcal{H}^{1}-\int_{\gamma^{\prime}}\right| f-\bar{f}\left|d \mathcal{H}^{1}\right|=\left|\int_{\left[X_{1}, X_{2}\right]}\left(|f-\bar{f}|\left(X, Y_{2}\right)-|f-\bar{f}|\left(X, Y_{1}\right)\right) d \mathcal{H}^{1}\right| \\
& \quad \leq \int_{\left[X_{1}, X_{2}\right]}\left|\left(|f-\bar{f}|\left(X, Y_{2}\right)-|f-\bar{f}|\left(X, Y_{1}\right)\right)\right| d \mathcal{H}^{1} \leq \int_{\left[X_{1}, X_{2}\right]}\left|\left((f-\bar{f})\left(X, Y_{2}\right)-(f-\bar{f})\left(X, Y_{1}\right)\right)\right| d \mathcal{H}^{1} \\
& \quad \leq \int_{\left[X_{1}, X_{2}\right]}\left|\int_{\left[Y_{1}, Y_{2}\right]} D_{2}(f-\bar{f}) d \mathcal{H}^{1}\right| d \mathcal{H}^{1} \leq \int_{D_{\gamma}}\left|D_{2}(f-\bar{f})\right| d X
\end{aligned}
$$

where $D_{2}$ denotes the distributional derivative in direction orthogonal to segment $\gamma$, and $\left(X_{1}, Y_{2}\right),\left(X_{2}, Y_{2}\right)$ are the endpoints of $\gamma,\left(X_{1}, Y_{1}\right),\left(X_{2}, Y_{1}\right)$ the endpoints of $\gamma^{\prime}$, see Fig. 9. Therefore,

$$
\int_{\gamma}|f-\bar{f}| d \mathcal{H}^{1} \leq \int_{\gamma^{\prime}}|f-\bar{f}| d \mathcal{H}^{1}+|D f|\left(D_{\gamma}\right) \leq \int_{\gamma_{1}}|f-\bar{f}| d \mathcal{H}^{1}+|D f|\left(B_{\epsilon}\right) \leq\left(C_{p 1}+1\right)|D f|\left(B_{\epsilon}\right),
$$

so that for $M$ segments, $(6.3)$ holds with $C_{t p^{\prime}}=M\left(C_{p 1}+1\right)$.

(S1) In this first step we show

$$
\begin{aligned}
& \left|D \boldsymbol{\varphi}_{\epsilon}\right|\left(\bigcup_{k=1}^{N_{\epsilon}} B_{c \epsilon}\left(\mathbf{X}_{k}\right)\right) \leq C_{2} \epsilon^{1 / 2}, \\
& \left|\mathbf{F}_{\epsilon}^{\mathrm{e}} \mathbf{F}_{\epsilon}^{\mathrm{p}}\right|\left(\bigcup_{k=1}^{N_{\epsilon}} B_{c \epsilon}\left(\mathbf{X}_{k}\right)\right) \leq C_{3} \epsilon^{1 / 2} .
\end{aligned}
$$

We start with (6.6). The displacement jump at $\mathbf{X} \in \mathcal{J}_{j} \cap B_{c \epsilon}\left(\mathbf{X}_{k}\right)$ is

$$
\llbracket \boldsymbol{\varphi}_{\epsilon}(\mathbf{X}) \rrbracket=\boldsymbol{\varphi}_{\epsilon}^{+}(\mathbf{X})-\boldsymbol{\varphi}_{\epsilon}^{-}(\mathbf{X})=\left(\boldsymbol{\varphi}_{\epsilon}^{+}(\mathbf{X})-\boldsymbol{\varphi}_{\epsilon}^{+}\left(\mathbf{X}_{k}\right)\right)-\left(\boldsymbol{\varphi}_{\epsilon}^{-}(\mathbf{X})-\boldsymbol{\varphi}_{\epsilon}^{-}\left(\mathbf{X}_{k}\right)\right)
$$

where we have used $\varphi_{\epsilon}^{+}\left(\mathbf{X}_{k}\right)=\varphi_{\epsilon}^{-}\left(\mathbf{X}_{k}\right)$, cf. (C3) in (5.4). We further note that between $\mathbf{X}_{k}$ and $\mathbf{X}$ either above or below $\mathcal{J}_{j}$ we do not cross any other jump set, and therefore $D \boldsymbol{\varphi}_{\epsilon}=\mathbf{F}_{\epsilon}^{\mathrm{e}} \mathcal{L}^{2}$ on both 
sides. Since $\mathbf{F}_{\epsilon}^{\mathrm{e}}$ has a trace on each side of the jump set, we obtain

$$
\varphi_{\epsilon}^{+}(\mathbf{X})-\varphi_{\epsilon}^{+}\left(\mathbf{X}_{k}\right)=\int_{\mathbf{X}_{k}}^{\mathbf{X}} \mathbf{F}_{\epsilon}^{\mathrm{e}+} d \mathbf{X}^{\prime},
$$

and the same on the other side. Inserting in (6.8),

$$
\boldsymbol{\varphi}_{\epsilon}^{+}(\mathbf{X})-\boldsymbol{\varphi}_{\epsilon}^{-}(\mathbf{X})=\int_{\mathbf{X}_{k}}^{\mathbf{X}}\left(\mathbf{F}_{\epsilon}^{\mathrm{e}+}-\mathbf{F}_{\epsilon}^{\mathrm{e}-}\right) d \mathbf{X}^{\prime},
$$

which implies $\left|\llbracket \boldsymbol{\varphi}_{\epsilon}(\mathbf{X}) \rrbracket\right| \leq\left|D \mathbf{F}_{\epsilon}^{\mathrm{e}}\right|\left(B_{c \epsilon}\left(\mathbf{X}_{k}\right)\right)$. Integrating over one of the slip lines inside the core, which have length $c \epsilon$, and then summing over all of the slip lines, we obtain

$$
\sum_{j=1}^{N_{d k}} \int_{\mathcal{J}_{j} \cap B_{c \epsilon}\left(\mathbf{X}_{k}\right)}\left|\llbracket \boldsymbol{\varphi}_{\epsilon} \rrbracket\right| d \mathcal{H}^{1} \leq N_{s} c \epsilon\left|D \mathbf{F}_{\epsilon}^{\mathrm{e}}\right|\left(B_{c \epsilon}\left(\mathbf{X}_{k}\right)\right),
$$

where we have used $N_{d k} \leq N_{s}$, cf. (C1) in (5.4). Summing now over all dislocation cores, we obtain

$$
\sum_{k=1}^{N_{\epsilon}} \sum_{j=1}^{N_{d k}} \int_{\mathcal{J}_{j} \cap B_{c \epsilon}\left(\mathbf{X}_{k}\right)}\left|\llbracket \boldsymbol{\varphi}_{\epsilon} \rrbracket\right| d \mathcal{H}^{1} \leq N_{s} c \epsilon\left|D \mathbf{F}_{\epsilon}^{\mathrm{e}}\right|\left(\bigcup_{k=1}^{N_{\epsilon}} B_{c \epsilon}\left(\mathbf{X}_{k}\right)\right) \leq C_{4} \epsilon E_{\epsilon} .
$$

We turn to the absolutely continuous part of the gradient of $\boldsymbol{\varphi}_{\epsilon}$, which by Hölder's inequality can be estimated as

$$
\sum_{k=1}^{N_{\epsilon}} \int_{B_{c \epsilon}\left(\mathbf{X}_{k}\right)}\left|\nabla \boldsymbol{\varphi}_{\epsilon}\right| d X=\sum_{k=1}^{N_{\epsilon}} \int_{B_{c \epsilon}\left(\mathbf{X}_{k}\right)}\left|\mathbf{F}_{\epsilon}^{\mathrm{e}}\right| d X \leq \sqrt{\pi} c \epsilon N_{\epsilon}^{1 / 2}\left(\sum_{k=1}^{N_{\epsilon}} \int_{B_{c \epsilon}\left(\mathbf{X}_{k}\right)}\left|\mathbf{F}_{\epsilon}^{\mathrm{e}}\right|^{2} d X\right)^{1 / 2} .
$$

Recalling the quadratic growth of the energy, cf. (5.3), and the definition (5.2) we see that the parenthesis is bounded. Since $N_{\epsilon} \leq C / \epsilon$ (recall (5.5)), this proves (6.6).

We now turn to (6.7). By (5.24) and the bound $\left|\mathbf{b}_{\epsilon j}\right| \leq n^{\prime} \epsilon \leq n \epsilon$, cf. (C2) and (C3) in (5.4),

$$
\left|\mathbf{F}_{\epsilon}^{\mathrm{e}} \mathbf{F}_{\epsilon}^{\mathrm{p}}\right|\left(B_{c \epsilon}\left(\mathbf{X}_{k}\right)\right) \leq \int_{B_{c \epsilon}\left(\mathbf{X}_{k}\right)}\left|\mathbf{F}_{\epsilon}^{\mathrm{e}}\right| d X+n \epsilon \sum_{j=1}^{N_{d k}} \int_{\mathcal{J}_{j} \cap B_{c \epsilon}\left(\mathbf{X}_{k}\right)}\left|\mathbf{F}_{\epsilon}^{\mathrm{e}}\right| d \mathcal{H}^{1} .
$$

In the second term we use (6.3), so that it becomes

$$
n \epsilon \sum_{j=1}^{N_{d k}} \int_{\mathcal{J}_{j} \cap B_{c \epsilon}\left(\mathbf{X}_{k}\right)}\left|\mathbf{F}_{\epsilon}^{\mathrm{e}}\right| d \mathcal{H}^{1} \leq n \epsilon \sum_{j=1}^{N_{d k}} \int_{\mathcal{J}_{j} \cap B_{c \epsilon}\left(\mathbf{X}_{k}\right)}\left|\overline{\mathbf{F}}_{\epsilon}^{\mathrm{e}}\right| d \mathcal{H}^{1}+C_{5} n \epsilon\left|D \mathbf{F}_{\epsilon}^{\mathrm{e}}\right|\left(B_{c \epsilon}\left(\mathbf{X}_{k}\right)\right),
$$

where $\overline{\mathbf{F}}_{\epsilon}^{\mathrm{e}}$ denotes the average of $\mathbf{F}_{\epsilon}^{\mathrm{e}}$ over $B_{c \epsilon}\left(\mathbf{X}_{k}\right)$, which obeys

$$
\left|\overline{\mathbf{F}}_{\epsilon}^{\mathrm{e}}\left(\mathbf{X}_{k}\right)\right|=\frac{1}{\pi c^{2} \epsilon^{2}}\left|\int_{B_{c \epsilon}\left(\mathbf{X}_{k}\right)} \mathbf{F}_{\epsilon}^{\mathrm{e}} d X\right| \leq \frac{1}{\pi c^{2} \epsilon^{2}} \int_{B_{c \epsilon}\left(\mathbf{X}_{k}\right)}\left|\mathbf{F}_{\epsilon}^{\mathrm{e}}\right| d X
$$

Therefore

$$
\left|\mathbf{F}_{\epsilon}^{\mathrm{e}} \mathbf{F}_{\epsilon}^{\mathrm{p}}\right|\left(B_{c \epsilon}\left(\mathbf{X}_{k}\right)\right) \leq\left(1+\frac{n N_{s} c}{\pi c^{2}}\right) \int_{B_{c \epsilon}\left(\mathbf{X}_{k}\right)}\left|\mathbf{F}_{\epsilon}^{\mathrm{e}}\right| d X+C_{5} n \epsilon\left|D \mathbf{F}_{\epsilon}^{\mathrm{e}}\right|\left(B_{c \epsilon}\left(\mathbf{X}_{k}\right)\right) .
$$

After summing over $k$, the first term is bounded as in (6.13), the second as in (6.12). This concludes the proof of (6.7). 
(S2) Next, we cover the domain $K \backslash \bigcup_{k=1}^{N_{\epsilon}} B_{c \epsilon}\left(\mathbf{X}_{k}\right)$ with finitely many balls $B_{\epsilon}\left(\mathbf{Y}_{l}\right)$, with $\mathbf{Y}_{l} \in K$, such that the larger balls $B_{L^{2} \epsilon}\left(\mathbf{Y}_{l}\right)$ do not intersect the dislocation cores $B_{m \epsilon}\left(\mathbf{X}_{k}\right)$ and have uniformly bounded overlap. Such a covering is possible in view of Eq. (5.6) and, by the definition of $K, B_{L^{2} \epsilon}\left(\mathbf{Y}_{l}\right) \subset \Omega^{\prime} \subset \Omega$. We first show that for any $l$ one has

$$
\left|D \boldsymbol{\varphi}_{\epsilon}-\mathbf{F}_{\epsilon}^{\mathrm{e}} \mathbf{F}_{\epsilon}^{\mathrm{p}}\right|\left(B_{\epsilon}\left(\mathbf{Y}_{l}\right)\right) \leq C_{6} \epsilon\left|D \mathbf{F}_{\epsilon}^{\mathrm{e}}\right|\left(B_{h \epsilon}\left(\mathbf{Y}_{l}\right)\right)
$$

with $h=1+L n \leq L^{2}$. Summing over all the balls will give the estimate on $K \backslash \bigcup_{k=1}^{N_{\epsilon}} B_{c \epsilon}\left(\mathbf{X}_{k}\right)$. Fix $l$ and let $\boldsymbol{\varphi}_{\epsilon}^{\mathrm{e}}$ and $\boldsymbol{\varphi}_{\epsilon}^{\mathrm{p}}$ be as in (C2), applied to the set $B_{2 L^{2} \epsilon}\left(\mathbf{Y}_{l}\right)$. Then, $\boldsymbol{\varphi}_{\epsilon}=\boldsymbol{\varphi}_{\epsilon}^{\mathrm{e}} \circ \boldsymbol{\varphi}_{\epsilon}^{\mathrm{p}}$. Taking the gradient we obtain

$$
D \boldsymbol{\varphi}_{\epsilon}=\mathbf{F}_{\epsilon}^{\mathrm{e}} \mathcal{L}^{2}+\sum_{j} \llbracket \boldsymbol{\varphi}_{\epsilon} \rrbracket \otimes \mathbf{N}_{j} \mathcal{H}^{1}\left\lfloor_{\mathcal{J}_{j}} \quad \text { and } \quad \mathbf{F}_{\epsilon}^{\mathrm{e}}=\tilde{\nabla} \boldsymbol{\varphi}_{\epsilon}^{\mathrm{e}} \circ \boldsymbol{\varphi}_{\epsilon}^{\mathrm{p}} \quad \text { in } B_{h \epsilon}\left(\mathbf{Y}_{l}\right)\right.
$$

Therefore, recalling (5.10),

$$
\left|D \boldsymbol{\varphi}_{\epsilon}-\mathbf{F}_{\epsilon}^{\mathrm{e}} \mathbf{F}_{\epsilon}^{\mathrm{p}}\right|\left(B_{\epsilon}\left(\mathbf{Y}_{l}\right)\right)=\int_{B_{\epsilon}\left(\mathbf{Y}_{l}\right) \cap \mathcal{J}}\left|\llbracket \boldsymbol{\varphi}_{\epsilon} \rrbracket-\mathbf{F}_{\epsilon}^{\mathrm{e}} \mathbf{b}_{\epsilon}\right| d \mathcal{H}^{1}
$$

where $\mathbf{b}_{\epsilon}$ takes the constant value $\mathbf{b}_{\epsilon j}$ on each segment $\mathcal{J}_{j} \subset B_{\epsilon}\left(\mathbf{Y}_{l}\right) \cap \mathcal{J}$. For $\mathbf{X} \in B_{\epsilon}\left(\mathbf{Y}_{l}\right) \cap \mathcal{J}_{j}$ we write $\tilde{\mathbf{X}}^{+}=\varphi_{\epsilon}^{\mathrm{p}+}(\mathbf{X}), \tilde{\mathbf{X}}^{-}=\varphi_{\epsilon}^{\mathrm{p}-}(\mathbf{X})$ and $\tilde{\mathbf{X}}^{+}-\tilde{\mathbf{X}}^{-}=\mathbf{b}_{\epsilon j}$, cf. (5.10). Then,

$$
\llbracket \boldsymbol{\varphi}_{\epsilon} \rrbracket(\mathbf{X})=\varphi_{\epsilon}^{\mathrm{e}}\left(\tilde{\mathbf{X}}^{+}\right)-\boldsymbol{\varphi}_{\epsilon}^{\mathrm{e}}\left(\tilde{\mathbf{X}}^{-}\right)=\int_{\tilde{\mathbf{X}}^{-}}^{\tilde{\mathbf{X}}^{+}} \tilde{\nabla} \boldsymbol{\varphi}_{\epsilon}^{\mathrm{e}} d \tilde{\mathbf{X}}=\int_{0}^{1} \mathbf{F}_{\epsilon}^{\mathrm{e}}\left(\boldsymbol{\varphi}_{\epsilon}^{\mathrm{p},-1}\left(\tilde{\mathbf{X}}^{-}+t \mathbf{b}_{\epsilon j}\right)\right) \mathbf{b}_{\epsilon j} d t .
$$

In the last step we used the second identity in (6.19). The value of $\mathbf{F}_{\epsilon}^{\mathrm{e}}$ on the jump set was defined in (5.24). Since $S=\left[\tilde{\mathbf{X}}^{+}, \tilde{\mathbf{X}}^{-}\right]$is a segment of length no larger than $n \epsilon$, by Lemma $5.1, \varphi_{\epsilon}^{\mathrm{p},-1}(S) \subset$ $B_{h \epsilon}\left(\mathbf{Y}_{l}\right)$. Furthermore, $\varphi_{\epsilon}^{\mathrm{p},-1}(S)$ consists of the union of at most $M=(1+n)^{N_{s}}$ segments. Indeed, each $\varphi_{\epsilon, \nu}^{\mathrm{p},-1}$ is piecewise a translation, and a segment of length no larger than $n \epsilon$ crosses at most $\lfloor n\rfloor+1$ discontinuity points, hence gets decomposed into at most $\lfloor n\rfloor+1$ segments, each of length no larger than $n \epsilon$ (plus possibly a finite number of points, which may be ignored). Iterating at most $N_{s}$ times gives the result. Then, by (6.3) one obtains

$$
\int_{\boldsymbol{\varphi}_{\epsilon}^{\mathrm{p},-1}(S)}\left|\mathbf{F}_{\epsilon}^{\mathrm{e}}-\overline{\mathbf{F}}_{\epsilon}^{\mathrm{e}}\right| d \mathcal{H}^{1} \leq C_{7}\left|D \mathbf{F}_{\epsilon}^{\mathrm{e}}\right|\left(B_{h \epsilon}\left(\mathbf{Y}_{l}\right)\right)
$$

where $\overline{\mathbf{F}}_{\epsilon}^{\mathrm{e}}$ is the average of $\mathbf{F}_{\epsilon}^{\mathrm{e}}$ over $B_{h \epsilon}\left(\mathbf{Y}_{l}\right)$,

$$
\overline{\mathbf{F}}_{\epsilon}^{\mathrm{e}}=\frac{1}{\left|B_{h \epsilon}\left(\mathbf{Y}_{l}\right)\right|} \int_{B_{h \epsilon}\left(\mathbf{Y}_{l}\right)} \mathbf{F}_{\epsilon}^{\mathrm{e}} d X
$$

Therefore (6.21) and (6.22) yield

$$
\begin{aligned}
\left|\llbracket \boldsymbol{\varphi}_{\epsilon} \rrbracket(\mathbf{X})-\overline{\mathbf{F}}_{\epsilon}^{\mathrm{e}} \mathbf{b}_{\epsilon j}\right| & =\left|\int_{0}^{1}\left(\mathbf{F}_{\epsilon}^{\mathrm{e}}\left(\boldsymbol{\varphi}_{\epsilon}^{\mathrm{p},-1}\left(\tilde{\mathbf{X}}^{-}+t \mathbf{b}_{\epsilon j}\right)\right)-\overline{\mathbf{F}}_{\epsilon}^{\mathrm{e}}\right) \mathbf{b}_{\epsilon j} d t\right| \\
& \leq \int_{\boldsymbol{\varphi}_{\epsilon}^{\mathrm{p},-1}(S)}\left|\mathbf{F}_{\epsilon}^{\mathrm{e}}-\overline{\mathbf{F}}_{\epsilon}^{\mathrm{e}}\right| d \mathcal{H}^{1} \leq C_{7}\left|D \mathbf{F}_{\epsilon}^{\mathrm{e}}\right|\left(B_{h \epsilon}\left(\mathbf{Y}_{l}\right)\right) .
\end{aligned}
$$

We write $(6.20)$ as

$$
\left|D \boldsymbol{\varphi}_{\epsilon}-\mathbf{F}_{\epsilon}^{\mathrm{e}} \mathbf{F}_{\epsilon}^{\mathrm{p}}\right|\left(B_{\epsilon}\left(\mathbf{Y}_{l}\right)\right) \leq \int_{B_{\epsilon}\left(\mathbf{Y}_{l}\right) \cap \mathcal{J}}\left|\llbracket \boldsymbol{\varphi}_{\epsilon} \rrbracket-\overline{\mathbf{F}}_{\epsilon}^{\mathrm{e}} \mathbf{b}_{\epsilon}\right| d \mathcal{H}^{1}+\left|\mathbf{b}_{\epsilon}\right| \int_{B_{\epsilon}\left(\mathbf{Y}_{l}\right) \cap \mathcal{J}}\left|\mathbf{F}_{\epsilon}^{\mathrm{e}}-\overline{\mathbf{F}}_{\epsilon}^{\mathrm{e}}\right| d \mathcal{H}^{1} .
$$

Since, by (6.3),

$$
\int_{B_{\epsilon}\left(\mathbf{Y}_{l}\right) \cap \mathcal{J}}\left|\mathbf{F}_{\epsilon}^{\mathrm{e}}-\overline{\mathbf{F}}_{\epsilon}^{\mathrm{e}}\right| d \mathcal{H}^{1} \leq C_{8}\left|D \mathbf{F}_{\epsilon}^{\mathrm{e}}\right|\left(B_{h \epsilon}\left(\mathbf{Y}_{l}\right)\right)
$$

this concludes the proof of (6.18). We remark that by $(\mathrm{C} 2)$ and the proof of Lemma $5.4,\left|B_{\epsilon}\left(\mathbf{Y}_{k}\right) \cap \mathcal{J}\right|$ consists of a finite union of segments bounded independently of $\epsilon$. 
(S3) We combine the results for the dislocation cores in (S1) and each $B_{\epsilon}\left(\mathbf{Y}_{l}\right)$ in (S2), which, by construction, have a finite overlap. Then,

$$
\left|D \varphi_{\epsilon}-\mathbf{F}_{\epsilon}^{\mathrm{e}} \mathbf{F}_{\epsilon}^{\mathrm{p}}\right|(K) \leq C_{1} \epsilon^{1 / 2}
$$

Next, we show the convergence of the mesoscopic quantities $\mathbf{F}^{\mathrm{e}}, \mathbf{F}_{\epsilon}^{\mathrm{e}}, \mathbf{F}_{\epsilon}^{\mathrm{p}}, \boldsymbol{\varphi}_{\epsilon}$ and Curl $\mathbf{F}_{\epsilon}^{\mathrm{p}}$ to their corresponding limiting quantities (which do not have the subindex $\epsilon$ ) in the appropriate topologies. At this stage the convergence of the elastic and plastic strains are independent, the relation between them will be treated later.

Theorem 6.2. Let $\boldsymbol{\varphi}_{\epsilon} \in X_{\epsilon}, \mathbf{F}_{\epsilon}, \mathbf{F}_{\epsilon}^{\mathrm{e}}$ and $\mathbf{F}_{\epsilon}^{\mathrm{p}}$ as defined in Section 5, and $\sup _{\epsilon} E_{\epsilon}\left(\boldsymbol{\varphi}_{\epsilon}\right)<\infty$. Then there exist $\mathbf{F}^{\mathrm{p}}, \mathbf{F}^{\mathrm{e}}, \boldsymbol{\varphi}$ and a subsequence (not relabeled) such that

$$
\begin{aligned}
& \mathbf{F}_{\epsilon}^{\mathrm{p}} \stackrel{*}{\rightarrow} \mathbf{F}^{\mathrm{p}} \quad \text { in } \mathcal{M}\left(\Omega ; \mathbb{R}^{2 \times 2}\right), \\
& \mathbf{F}_{\epsilon}^{\mathrm{e}} \rightarrow \mathbf{F}^{\mathrm{e}} \quad \text { in } L^{2}\left(\Omega ; \mathbb{R}^{2 \times 2}\right), \\
& \mathbf{F}_{\epsilon}^{\mathrm{e}} \stackrel{*}{\rightarrow} \mathbf{F}^{\mathrm{e}} \quad \text { in } B V\left(\Omega ; \mathbb{R}^{2 \times 2}\right), \\
& \mathbf{F}_{\epsilon}^{\mathrm{e}} \rightarrow \mathbf{F}^{\mathrm{e}} \quad \text { in } L^{1}\left(\Omega ; \mathbb{R}^{2 \times 2}\right), \\
& \boldsymbol{\varphi}_{\epsilon}-\mathbf{v}_{\epsilon} \rightarrow \boldsymbol{\varphi} \quad \text { in } L_{\mathrm{loc}}^{1}\left(\Omega ; \mathbb{R}^{2}\right), \\
& \mathbf{F}_{\epsilon}=D \boldsymbol{\varphi}_{\epsilon} \stackrel{*}{\rightarrow} D \boldsymbol{\varphi}=\mathbf{F} \quad \text { in } \mathcal{M}_{\mathrm{loc}}\left(\Omega ; \mathbb{R}^{2 \times 2}\right), \\
& \text { Curl } \mathbf{F}_{\epsilon}^{\mathrm{p}} \Omega_{\Omega^{\prime}} \stackrel{*}{\rightarrow} \text { Curl } \mathbf{F}^{\mathrm{p}} \quad \text { in } \mathcal{M}\left(\Omega ; \mathbb{R}^{2}\right)
\end{aligned}
$$

as $\epsilon \rightarrow 0$, where $\mathbf{v}_{\epsilon}$ is a sequence of rigid translations and $\Omega^{\prime}=\left\{\mathbf{X} \in \Omega: \operatorname{dist}(\mathbf{X}, \partial \Omega)>L^{2} \epsilon\right\}$. The support of the measure $\operatorname{Curl} \mathbf{F}_{\epsilon}^{\mathrm{p}}\left\lfloor_{\Omega^{\prime}}\right.$ thus exclusively consists of dislocation points. Furthermore $\boldsymbol{\varphi}$ is approximately continuous $\mathcal{H}^{1}$-almost everywhere.

We recall that $\mathcal{M}_{\text {loc }}\left(\Omega ; \mathbb{R}^{2}\right)$ is the space of vector-valued Radon measures on the set $\Omega, \mathcal{M}\left(\Omega ; \mathbb{R}^{2}\right)$ is the subset of bounded measures. A sequence $\mu_{\epsilon}$ is said to weakly* converge to $\mu$ in $\mathcal{M}(\Omega)$, and it is denoted $\stackrel{*}{\rightarrow}$, if

$$
\lim _{\epsilon \rightarrow 0} \int_{\Omega} f d \mu_{\epsilon}=\int_{\Omega} f d \mu
$$

for all continuous functions $f \in C^{0}(\Omega)$. Convergence in $\mathcal{M}_{\text {loc }}(\Omega)$ is the same if only functions $f \in C_{c}(\Omega)$ are allowed, cf. Definition 1.58 of Ambrosio et al. (2000).

\section{Proof. Convergence of $\mathbf{F}_{\epsilon}^{\mathrm{p}}$.}

By the definitions of $\mathbf{F}_{\epsilon}^{\mathrm{p}}$ and $\mathcal{J}$, and the bound on $\mathbf{b}_{\epsilon j}(5.29),\left|\mathbf{F}_{\epsilon}^{\mathrm{p}}\right|(\Omega) \leq \sqrt{2}|\Omega|+\sum_{j}\left|\mathbf{b}_{\epsilon j}\right|\left|\mathcal{J}_{j}\right| \leq$ $\sqrt{2}|\Omega|+n \epsilon|\mathcal{J}|$. By Lemma 5.4(ii) we obtain $\sup _{\epsilon}\left|\mathbf{F}_{\epsilon}^{\mathrm{p}}\right|(\Omega)<\infty$ and (6.29) follows by weak* compactness of finite Radon measures (Theorem 1.59 of Ambrosio et al. (2000)).

Convergence of $\mathbf{F}_{\epsilon}^{\mathrm{e}}$.

By the growth condition of the elastic energy (5.3), $\sup _{\epsilon}\left\|\mathbf{F}_{\epsilon}^{\mathrm{e}}\right\|_{L^{2}(\Omega)}<\infty$. Then, (6.30) follows by weak compactness (Theorem 1.36 of Ambrosio et al. (2000)). Furthermore, since $\Omega$ is bounded and the energy imposes $\sup _{\epsilon}\left|D \mathbf{F}_{\epsilon}^{\mathrm{e}}\right|(\Omega)<\infty$, we obtain $\sup _{\epsilon}\left\|\mathbf{F}_{\epsilon}^{\mathrm{e}}\right\|_{B V(\Omega)}<\infty$. Hence (6.31) and (6.32) follow by $B V$ compactness (Theorem 3.23 of Ambrosio et al. (2000)).

Convergence of $\varphi_{\epsilon}$ and $D \varphi_{\epsilon}$.

Consider a fixed closed ball $B$ compactly contained in $\Omega$, and define $\mathbf{v}_{\epsilon}=\frac{1}{|B|} \int_{B} \boldsymbol{\varphi}_{\epsilon} d X$. Next, choose $K^{\prime} \subset \subset \Omega$ and define $K=K^{\prime} \cup B$. We shall show that $\left|D \varphi_{\epsilon}\right|(K) \leq C(K)$ for all $\epsilon<\operatorname{dist}(K, \partial \Omega) /\left(2 L^{2}\right)$, with $C(K)$ depending only on $K$. To do this we decompose the domain into the dislocation cores and the remainder of the domain, as in the proof of Proposition 6.1. By (6.6),

$$
\left|D \boldsymbol{\varphi}_{\epsilon}\right|\left(\bigcup_{k=1}^{N_{\epsilon}} B_{c \epsilon}\left(\mathbf{X}_{k}\right)\right) \leq C_{2} \epsilon^{1 / 2}
$$


We cover $K \backslash \bigcup_{k=1}^{N_{\epsilon}} B_{c \epsilon}\left(\mathbf{X}_{k}\right)$ with balls of radius $\epsilon, B_{\epsilon}\left(\mathbf{Y}_{l}\right), l \in \mathbb{N}$ with finite overlap, as in Proposition 6.1 (S3) and we further define

$$
\overline{\mathbf{F}}_{\epsilon}^{\mathrm{e}}\left(\mathbf{Y}_{l}\right)=\frac{1}{\left|B_{\epsilon}\right|} \int_{B_{\epsilon}\left(\mathbf{Y}_{l}\right)} \mathbf{F}_{\epsilon}^{\mathrm{e}} d X
$$

By Proposition 6.1, $\left|D \boldsymbol{\varphi}_{\epsilon}-\mathbf{F}_{\epsilon}^{\mathrm{e}} \mathbf{F}_{\epsilon}^{\mathrm{p}}\right|(K) \leq C_{1} \epsilon^{1 / 2}$. The bound on $\left|\mathbf{F}_{\epsilon}^{\mathrm{e}} \mathbf{F}_{\epsilon}^{\mathrm{p}}\right|(K)$ is obtained with a similar strategy as in Proposition 6.1. For each ball, since $\left|\mathbf{b}_{\epsilon j}\right| \leq n \epsilon$ we have

$$
\left|\mathbf{F}_{\epsilon}^{\mathrm{e}} \mathbf{F}_{\epsilon}^{\mathrm{p}}\right|\left(B_{\epsilon}\left(\mathbf{Y}_{l}\right)\right) \leq \int_{B_{\epsilon}\left(\mathbf{Y}_{l}\right)}\left|\mathbf{F}_{\epsilon}^{\mathrm{e}}\right| d X+n \epsilon \int_{\mathcal{J} \cap B_{\epsilon}\left(\mathbf{Y}_{l}\right)}\left|\mathbf{F}_{\epsilon}^{\mathrm{e}}\right| d \mathcal{H}^{1},
$$

and $\mathcal{J} \cap B_{\epsilon}\left(\mathbf{Y}_{l}\right)$ consists of finitely many segments. Using (6.3) as in (6.27), and estimating the average via (6.16) as in (6.17) gives

$$
\left|\mathbf{F}_{\epsilon}^{\mathrm{e}} \mathbf{F}_{\epsilon}^{\mathrm{p}}\right|\left(B_{\epsilon}\left(\mathbf{Y}_{l}\right)\right) \leq C_{9} \int_{B_{\epsilon}\left(\mathbf{Y}_{l}\right)}\left|\mathbf{F}_{\epsilon}^{\mathrm{e}}\right| d X+C_{10} n \epsilon\left|D \mathbf{F}_{\epsilon}^{\mathrm{e}}\right|\left(B_{\epsilon}\left(\mathbf{Y}_{l}\right)\right) .
$$

Summing over all balls,

$$
\left|\mathbf{F}_{\epsilon}^{\mathrm{e}} \mathbf{F}_{\epsilon}^{\mathrm{p}}\right|\left(K \backslash\left(\bigcup_{k=1}^{N_{\epsilon}} B_{c \epsilon}\left(\mathbf{X}_{k}\right)\right)\right) \leq C_{11} \int_{\Omega}\left|\mathbf{F}_{\epsilon}^{\mathrm{e}}\right| d X+C_{12} \epsilon\left|D \mathbf{F}_{\epsilon}^{\mathrm{e}}\right|(\Omega)
$$

and therefore, recalling (6.37),

$$
\begin{aligned}
\left|D \boldsymbol{\varphi}_{\epsilon}\right|(K) & \leq\left|D \boldsymbol{\varphi}_{\epsilon}\right|\left(\bigcup_{k=1}^{N_{\epsilon}} B_{c \epsilon}\left(\mathbf{X}_{k}\right)\right)+\left|D \boldsymbol{\varphi}_{\epsilon}-\mathbf{F}_{\epsilon}^{\mathrm{e}} \mathbf{F}_{\epsilon}^{\mathrm{p}}\right|(K)+\left|\mathbf{F}_{\epsilon}^{\mathrm{e}} \mathbf{F}_{\epsilon}^{\mathrm{p}}\right|\left(K \backslash \bigcup_{k=1}^{N_{\epsilon}} B_{c \epsilon}\left(\mathbf{X}_{k}\right)\right) \\
& \leq C_{2} \epsilon^{1 / 2}+C_{1} \epsilon^{1 / 2}+C_{11} \int_{\Omega}\left|\mathbf{F}_{\epsilon}^{\mathrm{e}}\right| d X+\epsilon C_{12}\left|D \mathbf{F}_{\epsilon}^{\mathrm{e}}\right|(\Omega) .
\end{aligned}
$$

Therefore $\sup _{\epsilon}\left|D \varphi_{\epsilon}\right|(K)<\infty$. Then, by the Poincaré theorem for $B V$ functions, cf. Theorem 3.44 of Ambrosio et al. (2000), $\sup _{\epsilon}\left\|\boldsymbol{\varphi}_{\epsilon}-\bar{\varphi}_{\epsilon}\right\|_{L^{1}(K)}<\infty$, where $\bar{\varphi}_{\epsilon}$ is the average of $\boldsymbol{\varphi}_{\epsilon}$ over $K$. Since $B \subset K$ it follows that $\left|\mathbf{v}_{\epsilon}-\bar{\varphi}_{\epsilon}\right|$ is bounded as $\epsilon \rightarrow 0$. Then, as a result, by $B V$-compactness, cf. Theorem 3.23 of Ambrosio et al. (2000)) and the definition of weak* convergence in $B V$ (Definition 3.11 of Ambrosio et al. (2000)), (6.33) and (6.34) follow.

\section{Continuity of the limiting solution}

From the previous results, $\varphi_{\epsilon}-\mathbf{v}_{\epsilon} \rightarrow \varphi$ in $L_{\text {loc }}^{1}(\Omega)$. Further, the measures $D \varphi_{\epsilon}$ converge weakly to the measure $D \varphi$. Since the total variation of a limit is smaller or equal than the limit of the total variations, see for example page 172 of Evans and Gariepy (1991),

$$
|D \varphi|\left(B_{r}\right) \leq \liminf _{\epsilon \rightarrow 0}\left|D \varphi_{\epsilon}\right|\left(B_{r}\right) \quad \forall B_{r} \subset \subset \Omega,
$$

where $B_{r}$ denotes a ball of radius $r$. We write $B_{2 r}$ for the ball with the same center and radius $2 r$, and assume $B_{2 r} \subset \subset \Omega$.

Let us consider a set $K \subset \subset \Omega$, such that $B_{2 r} \subset \subset K$, and take $\epsilon<\operatorname{dist}(\partial \Omega, K) /\left(2 L^{2}\right)$. Then, defining $K_{\epsilon}=K \backslash\left(\bigcup_{k=1}^{N_{\epsilon}} B_{c \epsilon}\left(\mathbf{X}_{k}\right)\right)$, we obtain by Proposition 6.1 and $(6.37)$

$$
\begin{aligned}
\left|D \boldsymbol{\varphi}_{\epsilon}\right|\left(B_{r}\right) & \leq\left|D \boldsymbol{\varphi}_{\epsilon}\right|\left(B_{r} \cap K_{\epsilon}\right)+\left|D \boldsymbol{\varphi}_{\epsilon}\right|\left(B_{r} \cap \bigcup_{k=1}^{N_{\epsilon}} B_{c \epsilon}\left(\mathbf{X}_{k}\right)\right) \\
& \leq\left|D \boldsymbol{\varphi}_{\epsilon}-\mathbf{F}_{\epsilon}^{\mathrm{e}} \mathbf{F}_{\epsilon}^{\mathrm{p}}\right|\left(B_{r} \cap K_{\epsilon}\right)+\left|\mathbf{F}_{\epsilon}^{\mathrm{e}} \mathbf{F}_{\epsilon}^{\mathrm{p}}\right|\left(B_{r} \cap K_{\epsilon}\right)+\left|D \boldsymbol{\varphi}_{\epsilon}\right|\left(B_{r} \cap \bigcup_{k=1}^{N_{\epsilon}} B_{c \epsilon}\left(\mathbf{X}_{k}\right)\right) \\
& \leq C_{13} \epsilon^{1 / 2}+\left|\mathbf{F}_{\epsilon}^{\mathrm{e}} \mathbf{F}_{\epsilon}^{\mathrm{p}}\right|\left(B_{r} \cap K_{\epsilon}\right) .
\end{aligned}
$$


To estimate the last term we cover $B_{r} \cap K_{\epsilon}$ with balls of radius $\epsilon$ which do not intersect the cores, as done in (6.41). The balls have finite overlap and are all contained in the larger ball $B_{2 r}$. Therefore

$$
\left|D \boldsymbol{\varphi}_{\epsilon}\right|\left(B_{r}\right) \leq C_{13} \epsilon^{1 / 2}+C_{14}\left\|\mathbf{F}_{\epsilon}^{\mathrm{e}}\right\|_{L^{1}\left(B_{2 r} \cap K_{\epsilon}\right)}+C_{15} \epsilon\left|D \mathbf{F}_{\epsilon}^{\mathrm{e}}\right|\left(B_{2 r}\right) .
$$

Passing to the limit, since (6.32) implies $\left\|\mathbf{F}_{\epsilon}^{\mathrm{e}}\right\|_{L^{1}\left(B_{2 r}\right)} \rightarrow\left\|\mathbf{F}^{\mathrm{e}}\right\|_{L^{1}\left(B_{2 r}\right)}$, we obtain

$$
|D \boldsymbol{\varphi}|\left(B_{r}\right) \leq \liminf _{\epsilon \rightarrow 0}\left|D \boldsymbol{\varphi}_{\epsilon}\right|\left(B_{r}\right) \leq C_{14}\left\|\mathbf{F}^{\mathrm{e}}\right\|_{L^{1}\left(B_{2 r}\right)} \leq C_{14} 2 \sqrt{\pi} r\left\|\mathbf{F}^{\mathrm{e}}\right\|_{L^{2}\left(B_{2 r}\right)} .
$$

Therefore

$$
|D \varphi|\left(B_{r}\right) \leq C_{16} r\left\|\mathbf{F}^{\mathrm{e}}\right\|_{L^{2}\left(B_{2 r}\right)}
$$

Since, by $(6.30), \mathbf{F}^{\mathrm{e}} \in L^{2}(\Omega)$,

$$
\liminf _{r \rightarrow 0} \frac{|D \varphi|\left(B_{r}\right)}{r}=0
$$

As a result, cf. Proposition 3.92 of Ambrosio et al. (2000), the jump set of $D \varphi$ has $\mathcal{H}^{1}$ measure zero, and $\boldsymbol{\varphi}$ is approximately continuous $\mathcal{H}^{1}$-almost everywhere. We note that it may contain a Cantor part.

\section{Convergence of Curl $\mathbf{F}_{\epsilon}^{\mathrm{p}}$}

By the scaling (5.5) and the bound on the Burgers vector (5.29), Curl $\mathbf{F}_{\epsilon}^{\mathrm{p}}\left\lfloor_{\Omega^{\prime}}\right.$ is equibounded. Therefore, by weak* compactness (Theorem 1.59 of Ambrosio et al. (2000)),

$$
\operatorname{Curl} \mathbf{F}_{\epsilon}^{\mathrm{p}}\left\lfloor\Omega^{\prime} \stackrel{*}{\rightarrow} \mathbf{G} \quad \text { in } \mathcal{M}\left(\Omega ; \mathbb{R}^{2}\right)\right. \text {. }
$$

Since $\mathbf{F}_{\epsilon}^{\mathrm{p}}$ also converges weakly* in $\mathcal{M}$, we have $\mathbf{G}=\operatorname{Curl} \mathbf{F}^{\mathrm{p}}$. Indeed, for every $\phi \in C_{c}^{\infty}(\Omega)$, one can choose $\epsilon$ such that supp $\phi \subset \subset \Omega^{\prime}$. Then, cf. Appendix A,

$$
\lim _{\epsilon \rightarrow 0} \int_{\Omega} \phi d\left(\operatorname{Curl} \mathbf{F}_{\epsilon}^{\mathrm{p}}-\operatorname{Curl} \mathbf{F}^{\mathrm{p}}\right)=\lim _{\epsilon \rightarrow 0} \int_{\Omega^{\prime}} \phi d\left(\operatorname{Curl} \mathbf{F}_{\epsilon}^{\mathrm{p}}-\operatorname{Curl} \mathbf{F}^{\mathrm{p}}\right)=\lim _{\epsilon \rightarrow 0}-\int_{\Omega^{\prime}} D \phi \times d\left(\mathbf{F}_{\epsilon}^{\mathrm{p}}-\mathbf{F}^{\mathrm{p}}\right)=0
$$

where the cross product is considered between $D \phi$ and each row of $\left(\mathbf{F}_{\epsilon}^{\mathrm{p}}-\mathbf{F}^{\mathrm{p}}\right)$.

The next assertion is our main result, stating that the gradient of the limit deformation $\varphi$ (which is defined as the limit of the deformations) coincides with the product of the elastic strain $\mathbf{F}^{\mathrm{e}}$ (defined as the limit of the mesoscopic elastic strains $\mathbf{F}_{\epsilon}^{\mathrm{e}}$ ) and the plastic strain $\mathbf{F}^{\mathrm{p}}$ (defined as the limit of the measure-valued mesoscopic plastic strains $\mathbf{F}_{\epsilon}^{\mathrm{p}}$ ).

Theorem 6.3. Let $\boldsymbol{\varphi}_{\epsilon}, \mathbf{F}_{\epsilon}=D \boldsymbol{\varphi}_{\epsilon}, \mathbf{F}_{\epsilon}^{\mathrm{e}}, \mathbf{F}_{\epsilon}^{\mathrm{p}}$ as defined in Section 5, with $\sup _{\epsilon} E_{\epsilon}\left(\boldsymbol{\varphi}_{\epsilon}\right)<\infty$. Let $\mathbf{F}=D \varphi$, $\mathbf{F}^{\mathrm{e}}$ and $\mathbf{F}^{\mathrm{p}}$ be as in Theorem 6.2. Then

$$
\mathbf{F}=\mathbf{F}^{\mathrm{e}} \mathbf{F}^{\mathrm{p}}
$$

Additionally, $\mathbf{F}^{\mathrm{p}} \in L^{\infty}\left(\Omega ; \mathbb{R}^{2 \times 2}\right), \mathbf{F} \in L^{2}\left(\Omega ; \mathbb{R}^{2 \times 2}\right)$ and $\boldsymbol{\varphi} \in W^{1,2}\left(\Omega ; \mathbb{R}^{2}\right)$.

Proof. We first show $D \boldsymbol{\varphi}_{\epsilon} \stackrel{*}{\rightarrow} \mathbf{F}^{\mathrm{e}} \mathbf{F}^{\mathrm{p}}$ in the sense of distributions. By Theorem 6.2 and uniqueness of the limit, this implies (6.51).

We begin by defining $\boldsymbol{\mu}_{\epsilon}$ as the mollification of $\mathbf{F}_{\epsilon}^{\mathrm{p}}$ with a kernel $\eta_{\epsilon} \in C_{c}^{\infty}\left(B_{\epsilon}\right)$ satisfying $\int_{B_{\epsilon}} \eta_{\epsilon} d X=1$, $\eta_{\epsilon}(X)=\eta_{\epsilon}(-X)$ and $\eta_{\epsilon} \leq C_{17} \frac{1}{\epsilon^{2}} \chi_{B_{\epsilon}}$, i.e.

$$
\boldsymbol{\mu}_{\epsilon}(\mathbf{X})=\left(\mathbf{F}_{\epsilon}^{\mathrm{p}} * \eta_{\epsilon}\right)(\mathbf{X})=\int_{B_{\epsilon}} \eta_{\epsilon}(\mathbf{X}-\mathbf{Y}) d \mathbf{F}_{\epsilon}^{\mathrm{p}}(\mathbf{Y})
$$

Then, for any $K \subset \subset \Omega$, and $\epsilon<\operatorname{dist}(\partial \Omega, K) /\left(2 L^{2}\right)$, one has, by (5.52) and (5.29), that $\left\|\boldsymbol{\mu}_{\epsilon}\right\|_{L^{\infty}(K)}$ is equibounded, the bound does not depend on $K$. Therefore there exist $\boldsymbol{\mu} \in L^{\infty}\left(\Omega ; \mathbb{R}^{2 \times 2}\right)$ and a subsequence 
satisfying $\boldsymbol{\mu}_{\epsilon} \stackrel{*}{\rightarrow} \boldsymbol{\mu}$ in $L^{\infty}\left(K ; \mathbb{R}^{2 \times 2}\right)$ for all $K \subset \subset \Omega$. Next, we show that $\boldsymbol{\mu}=\mathbf{F}^{\mathrm{p}}$. Let $\phi \in C_{c}^{\infty}(\Omega)$. By choosing $K$ such that $\operatorname{supp}(\phi) \subset \subset K \subset \subset \Omega$, and choosing $\epsilon<\operatorname{dist}(\partial \Omega, K) /\left(2 L^{2}\right)$,

$$
\begin{aligned}
\int_{\Omega} \phi\left(d \mathbf{F}_{\epsilon}^{\mathrm{p}}-d \boldsymbol{\mu}_{\epsilon}\right) & =\int_{K} \phi\left(d \mathbf{F}_{\epsilon}^{\mathrm{p}}-d \boldsymbol{\mu}_{\epsilon}\right)=\int_{K} \phi\left(d \mathbf{F}_{\epsilon}^{\mathrm{p}}-d\left(\mathbf{F}_{\epsilon}^{\mathrm{p}} * \eta_{\epsilon}\right)\right)=\int_{K}\left(\phi-\phi * \eta_{\epsilon}\right) d \mathbf{F}_{\epsilon}^{\mathrm{p}} \\
& \leq \sup _{\epsilon}\left|\phi-\phi * \eta_{\epsilon}\right|\left|\mathbf{F}_{\epsilon}^{\mathrm{p}}\right|(\Omega) \rightarrow 0, \text { as } \epsilon \rightarrow 0,
\end{aligned}
$$

since $\left|\mathbf{F}_{\epsilon}^{\mathrm{p}}\right|(\Omega)$ equibounded, cf. proof of Theorem 6.2, and $\phi * \eta_{\epsilon} \rightarrow \phi$ uniformly as $\epsilon \rightarrow 0$. Therefore $\boldsymbol{\mu}=\mathbf{F}^{\mathrm{p}}$ and $\mathbf{F}^{\mathrm{p}} \in L^{\infty}\left(\Omega ; \mathbb{R}^{2 \times 2}\right)$. Furthermore, writing for simplicity, with abuse of notation, $D \boldsymbol{\varphi}_{\epsilon} d X$ and $\mathbf{F}_{\epsilon}^{\mathrm{p}} d X$ for $d D \boldsymbol{\varphi}_{\epsilon}$ and $d \mathbf{F}_{\epsilon}^{\mathrm{p}}$,

$$
\begin{aligned}
\left|\int_{\Omega} \phi\left(D \boldsymbol{\varphi}_{\epsilon}-\mathbf{F}^{\mathrm{e}} \mathbf{F}^{\mathrm{p}}\right) d X\right|= & \left|\int_{K} \phi\left(D \boldsymbol{\varphi}_{\epsilon}-\mathbf{F}^{\mathrm{e}} \mathbf{F}^{\mathrm{p}}\right) d X\right| \\
\leq & \int_{K}\left|\phi\left(D \boldsymbol{\varphi}_{\epsilon}-\mathbf{F}_{\epsilon}^{\mathrm{e}} \mathbf{F}_{\epsilon}^{\mathrm{p}}\right)\right| d X+\left|\int_{K} \phi\left(\mathbf{F}_{\epsilon}^{\mathrm{e}} \mathbf{F}_{\epsilon}^{\mathrm{p}}-\mathbf{F}_{\epsilon}^{\mathrm{e}} \boldsymbol{\mu}_{\epsilon}\right) d X\right| \\
& +\int_{K}\left|\phi\left(\mathbf{F}_{\epsilon}^{\mathrm{e}} \boldsymbol{\mu}_{\epsilon}-\mathbf{F}^{\mathrm{e}} \boldsymbol{\mu}_{\epsilon}\right)\right| d X+\left|\int_{K} \phi\left(\mathbf{F}^{\mathrm{e}} \boldsymbol{\mu}_{\epsilon}-\mathbf{F}^{\mathrm{e}} \mathbf{F}^{\mathrm{p}}\right) d X\right| .
\end{aligned}
$$

The first term, $\int_{K}\left|\phi\left(D \boldsymbol{\varphi}_{\epsilon}-\mathbf{F}_{\epsilon}^{\mathrm{e}} \mathbf{F}_{\epsilon}^{\mathrm{p}}\right)\right| d X$, tends to zero by Proposition 6.1. The third term, $\int_{K}\left|\phi\left(\mathbf{F}_{\epsilon}^{\mathrm{e}} \boldsymbol{\mu}_{\epsilon}-\mathbf{F}^{\mathrm{e}} \boldsymbol{\mu}_{\epsilon}\right)\right| d X$, tends to zero by the strong $L^{1}(K)$ convergence of $\mathbf{F}_{\epsilon}^{\mathrm{e}}$, cf. (6.32), and $\boldsymbol{\mu}_{\epsilon} \in L^{\infty}(K)$. The last term, $\left|\int_{K} \phi\left(\mathbf{F}^{\mathrm{e}} \boldsymbol{\mu}_{\epsilon}-\mathbf{F}^{\mathrm{e}} \mathbf{F}^{\mathrm{p}}\right) d X\right|$, tends to zero since $\phi \mathbf{F}^{\mathrm{e}} \in L^{1}(K)$ and $\boldsymbol{\mu}_{\epsilon}$ weakly* converges to $\mathbf{F}^{\mathrm{p}}$ in $L^{\infty}(K)$. Finally, in the second term, $\left|\int_{K} \phi\left(\mathbf{F}_{\epsilon}^{\mathrm{e}} \mathbf{F}_{\epsilon}^{\mathrm{p}}-\mathbf{F}_{\epsilon}^{\mathrm{e}} \boldsymbol{\mu}_{\epsilon}\right) d X\right|$, we add and subtract the identity, use $\mathbf{I} * \eta_{\epsilon}=\mathbf{I}$ and a change of variables in the second term, to get

$$
\begin{aligned}
\int_{K} \phi\left(\mathbf{F}_{\epsilon}^{\mathrm{e}} \mathbf{F}_{\epsilon}^{\mathrm{p}}-\mathbf{F}_{\epsilon}^{\mathrm{e}} \boldsymbol{\mu}_{\epsilon}\right) d X & =\int_{K}\left[\phi \mathbf{F}_{\epsilon}^{\mathrm{e}}\left(\mathbf{F}_{\epsilon}^{\mathrm{p}}-\mathbf{I}\right)-\phi \mathbf{F}_{\epsilon}^{\mathrm{e}}\left(\left(\mathbf{F}_{\epsilon}^{\mathrm{p}}-\mathbf{I}\right) * \eta_{\epsilon}\right)\right] d X \\
& =\int_{K}\left[\phi \mathbf{F}_{\epsilon}^{\mathrm{e}}\left(\mathbf{F}_{\epsilon}^{\mathrm{p}}-\mathbf{I}\right)-\left(\left(\phi \mathbf{F}_{\epsilon}^{\mathrm{e}}\right) * \eta_{\epsilon}\right)\left(\mathbf{F}_{\epsilon}^{\mathrm{p}}-\mathbf{I}\right)\right] d X \\
& =\int_{K \cap \mathcal{J}}\left[\phi \mathbf{F}_{\epsilon}^{\mathrm{e}}-\left(\phi \mathbf{F}_{\epsilon}^{\mathrm{e}}\right) * \eta_{\epsilon}\right] \mathbf{b}_{\epsilon} \otimes \mathbf{N} d \mathcal{H}^{1} .
\end{aligned}
$$

We cover the jump set $\mathcal{J}$ with balls $B_{\epsilon}\left(\mathbf{Y}_{l}\right)$ such that the double balls $B_{2 \epsilon}\left(\mathbf{Y}_{l}\right)$ are contained in $\Omega$ and have finite overlap. We use (6.3) in each ball, both for $\phi \mathbf{F}_{\epsilon}^{\mathrm{e}}$ and for $\left(\phi \mathbf{F}_{\epsilon}^{\mathrm{e}}\right) * \eta_{\epsilon}$, with $\overline{\phi \mathbf{F}_{\epsilon}^{\mathrm{e}}}$ and $\overline{\phi \mathbf{F}_{\epsilon}^{\mathrm{e}} * \eta_{\epsilon}}$ the averages of $\phi \mathbf{F}_{\epsilon}^{\mathrm{e}}$ and $\phi \mathbf{F}_{\epsilon}^{\mathrm{e}} * \eta_{\epsilon}$ over $B_{2 \epsilon}\left(\mathbf{Y}_{l}\right)$, and the fact that by Poincaré's inequality $\epsilon^{2}\left|\overline{\phi \mathbf{F}_{\epsilon}^{\mathrm{e}}}-\overline{\phi \mathbf{F}_{\epsilon}^{\mathrm{e}} * \eta_{\epsilon}}\right| \leq$ $C \epsilon\left|D\left(\phi \mathbf{F}_{\epsilon}^{\mathrm{e}}\right)\right|\left(B_{2 \epsilon}\left(\mathbf{Y}_{l}\right)\right)$ to obtain

$$
\begin{aligned}
\left|\int_{K} \phi\left(\mathbf{F}_{\epsilon}^{\mathrm{e}} \mathbf{F}_{\epsilon}^{\mathrm{p}}-\mathbf{F}_{\epsilon}^{\mathrm{e}} \boldsymbol{\mu}_{\epsilon}\right) d X\right| & \leq \sum_{l} n \epsilon \int_{B_{\epsilon}\left(\mathbf{Y}_{l}\right) \cap \mathcal{J}}\left|\phi \mathbf{F}_{\epsilon}^{\mathrm{e}}-\left(\phi \mathbf{F}_{\epsilon}^{\mathrm{e}}\right) * \eta_{\epsilon}\right| d \mathcal{H}^{1} \\
& \leq \sum_{l} n \epsilon C_{18}\left|D\left(\phi \mathbf{F}_{\epsilon}^{\mathrm{e}}\right)\right|\left(B_{2 \epsilon}\left(\mathbf{Y}_{l}\right)\right) \\
& \leq C_{19} n \epsilon\left(\|\phi\|_{L^{\infty}(\Omega)}\left|D \mathbf{F}_{\epsilon}^{\mathrm{e}}\right|(\Omega)+\|D \phi\|_{L^{\infty}(\Omega)} \int_{\Omega}\left|\mathbf{F}_{\epsilon}^{\mathrm{e}}\right| d \mathbf{X}\right) \leq C_{20} \epsilon,
\end{aligned}
$$

where we have further used the product rule, the quadratic growth of the elastic energy and the energy bound. Therefore $D \boldsymbol{\varphi}_{\epsilon}$ converges distributionally to $\mathbf{F}^{\mathrm{e}} \mathbf{F}^{\mathrm{p}}$. By uniqueness of the limit, then $\mathbf{F}=\mathbf{F}^{\mathrm{e}} \mathbf{F}^{\mathrm{p}}$. Further, since $\mathbf{F}^{\mathrm{e}} \in L^{2}\left(\Omega ; \mathbb{R}^{2 \times 2}\right)$, and $\mathbf{F}^{\mathrm{p}} \in L^{\infty}\left(\Omega ; \mathbb{R}^{2 \times 2}\right)$, it immediately follows that $\mathbf{F} \in L^{2}\left(\Omega ; \mathbb{R}^{2 \times 2}\right)$. By the Poincaré inequality, $\varphi-\bar{\varphi} \in L^{2}\left(\Omega ; \mathbb{R}^{2}\right)$. Since $\Omega$ is bounded, also $\varphi \in L^{2}\left(\Omega ; \mathbb{R}^{2}\right)$.

Our last result shows that the limiting plastic strain $\mathbf{F}^{\mathrm{p}}$ satisfies $\operatorname{det} \mathbf{F}^{\mathrm{p}}=1$. This is highly nontrivial since the mesoscopic plastic strains $\mathbf{F}_{\epsilon}^{\mathrm{p}}$ are singular and, therefore, possess no determinant in a classical sense; 
at the same time the convergence is only weakly in measures and does not, in general, lead to convergence of the determinants. We therefore resort to the detailed structure of the mesoscopic deformation, whose plastic part was volume-preserving, and to a suitable iterative mollification scheme to defined regularized determinants.

Theorem 6.4. Let $\boldsymbol{\varphi}_{\epsilon}$ as defined in Section 5, with $\sup _{\epsilon} E_{\epsilon}\left(\boldsymbol{\varphi}_{\epsilon}\right)<\infty, \mathbf{F}^{\mathrm{p}}$ as in Theorem 6.2. Then $\operatorname{det} \mathbf{F}^{\mathrm{p}}=1$.

Proof. The strategy for the proof consists in constructing a mollified version of $\mathbf{F}_{\epsilon}^{\mathrm{p}}$, denoted by $\mathbf{F}_{\epsilon \alpha}^{\mathrm{p}}$, starting from the representation of the mesoscopic deformation as a local concatenation of slips. Since the individual slips are volume-preserving, the determinant of $\mathbf{F}_{\epsilon \alpha}^{\mathrm{p}}$ is exactly one away from the dislocations. One key difficulty is that this definition is local, and care needs to be taken at the interface between different patches. Once this is done, a global definition is obtained, and it only remains to show that $\mathbf{F}_{\epsilon \alpha}^{\mathrm{p}}$ and its determinant converge to $\mathbf{F}^{\mathrm{p}}$ and $\operatorname{det} \mathbf{F}^{\mathrm{p}}$, respectively.

Consider the set $\Omega_{2}^{\prime}=\left\{\mathbf{X} \in \Omega: \operatorname{dist}(\mathbf{X}, \partial \Omega) \geq 2 L^{2} \epsilon\right\}$, with $L$ as defined in Lemma 5.1. Next, we cover $\Omega_{2}^{\prime} \backslash \bigcup_{k=1}^{N_{\epsilon}} B_{c \epsilon}\left(\mathbf{X}_{k}\right)$ with balls $B_{\epsilon}\left(\mathbf{Y}_{l}\right)$, such that $\mathbf{Y}_{l} \in \Omega_{2}^{\prime}$ and $\left|\mathbf{Y}_{l}-\mathbf{X}_{k}\right|>c \epsilon$ for all $l, k$. Note that this cover is possible by the constraint on the minimum distance between dislocations, cf. Eq. (5.6), and our choice of $\Omega_{2}^{\prime}$. We fix a mollification kernel $\eta_{\alpha} \in C_{c}^{\infty}\left(B_{\alpha}\right)$, where $\alpha=\epsilon / 10$. In $B_{2 L^{2} \epsilon}\left(\mathbf{Y}_{l}\right)$ we choose a decomposition as in $(\mathrm{C} 2), \varphi_{\epsilon}^{\mathrm{p}}=\varphi_{\epsilon, N_{s}}^{\mathrm{p}} \circ \ldots \circ \varphi_{\epsilon, 1}^{\mathrm{p}}$. We define, in the smaller ball $B_{\epsilon}\left(\mathbf{Y}_{l}\right)$, the regularized plastic deformation by

$$
\varphi_{\epsilon \alpha}^{\mathrm{p}}=\left(\varphi_{\epsilon, N_{s}}^{\mathrm{p}} * \eta_{\alpha}\right) \circ \ldots \circ\left(\varphi_{\epsilon, \nu}^{\mathrm{p}} * \eta_{\alpha}\right) \circ \ldots \circ\left(\boldsymbol{\varphi}_{\epsilon, 1}^{\mathrm{p}} * \eta_{\alpha}\right)
$$

We observe that $\varphi_{\epsilon \alpha}^{\mathrm{p}}$ is smooth, and $\operatorname{det} D\left(\boldsymbol{\varphi}_{\epsilon, \nu}^{\mathrm{p}} * \eta_{\alpha}\right)=1$ implies $\operatorname{det} D \boldsymbol{\varphi}_{\epsilon \alpha}^{\mathrm{p}}=1$ everywhere. Indeed, by the chain rule the gradient of the composition is the product of the gradients (evaluated at appropriate points); standard properties of the determinants show that the determinant of the product is the product of the determinants. Further, since the jumps of each $\varphi_{\epsilon, \nu}^{\mathrm{p}}$ are bounded by $n^{\prime} \epsilon$ and separated by at least $\epsilon$, cf. (C2), we obtain $\left|\varphi_{\epsilon, \nu}^{\mathrm{p}} * \eta_{\alpha}-\varphi_{\epsilon, \nu}^{\mathrm{p}}\right| \leq n^{\prime} \epsilon$ and $\left|D\left(\varphi_{\epsilon, \nu}^{\mathrm{p}} * \eta_{\alpha}\right)\right| \leq c^{\prime} n^{\prime} \epsilon / \alpha$ for some constant $c^{\prime}$. Iterating we deduce that $\left|\varphi_{\epsilon \alpha}^{\mathrm{p}}-\varphi_{\epsilon}^{\mathrm{p}}\right| \leq C_{21} \epsilon$ and $\left|D \varphi_{\epsilon \alpha}^{\mathrm{p}}\right| \leq C_{22}$.

We now show that $D \varphi_{\epsilon \alpha}^{\mathrm{p}}$ is uniquely defined, independently on the choice of $l$ and the decomposition of $\varphi_{\epsilon}^{\mathrm{p}}$. Let $\hat{\varphi}_{\epsilon}^{\mathrm{p}}=\hat{\varphi}_{\epsilon, N_{s}}^{\mathrm{p}} \circ \ldots \circ \hat{\varphi}_{\epsilon, 1}^{\mathrm{p}}$ be a different decomposition. By Lemma 5.3, there are constants $\mathbf{c}_{\nu}$ such that $\boldsymbol{\varphi}_{\epsilon, \nu}^{\mathrm{p}}(\mathbf{X})=\hat{\boldsymbol{\varphi}}_{\epsilon, \nu}^{\mathrm{p}}\left(\mathbf{X}-\mathbf{c}_{\nu-1}\right)+\mathbf{c}_{\nu}$, which implies $\left(\boldsymbol{\varphi}_{\epsilon, \nu}^{p} * \eta_{\alpha}\right)(\mathbf{X})=\left(\hat{\boldsymbol{\varphi}}_{\epsilon, \nu}^{\mathrm{p}} * \eta_{\alpha}\right)\left(\mathbf{X}-\mathbf{c}_{\nu-1}\right)+\mathbf{c}_{\nu}$. Iterating as in (5.49-5.50) gives $D \varphi_{\epsilon \alpha}^{\mathrm{p}}=D \hat{\varphi}_{\epsilon \alpha}^{\mathrm{p}}$. We define $\mathbf{F}_{\epsilon \alpha}^{\mathrm{p}}$ as $D \varphi_{\epsilon \alpha}^{\mathrm{p}}$ on $\cup_{l} B_{\epsilon}\left(\mathbf{Y}_{l}\right)$ and zero elsewhere.

Next, we choose a constant $1 / 2<\rho<1$, and define $\Omega^{\prime \prime}=\left\{\mathbf{X} \in \Omega\right.$ : $\left.\operatorname{dist}(\mathbf{X}, \partial \Omega) \geq \epsilon^{\rho}\right\}$. We now construct a cover of $\Omega^{\prime \prime}$ with balls of radius of order $\epsilon^{\rho}$. Precisely, we first select balls $B_{\epsilon} \rho\left(\mathbf{X}_{k}\right)$ centered at the dislocation points, and then finitely many $\mathbf{Y}_{l} \in H=\left\{\mathbf{X} \in \Omega: \operatorname{dist}(\mathbf{X}, \partial \Omega) \geq \epsilon^{\rho} / 2\right\} \backslash \bigcup_{k=1}^{N_{\epsilon}} B_{\epsilon^{\rho} / 2}\left(\mathbf{X}_{k}\right)$ such that $H \subset \cup_{l} B_{\epsilon^{\rho} / 4}\left(\mathbf{Y}_{l}\right)$ (this is possible since $H$ is compact). Let $\phi_{k}^{\prime} \in C_{c}^{\infty}\left(B_{\epsilon^{\rho} / 2}\left(\mathbf{X}_{k}\right)\right), \phi_{l} \in C_{c}^{\infty}\left(B_{\epsilon^{\rho} / 4}\left(\mathbf{Y}_{l}\right)\right)$ be a partition of unity on $\left\{\mathbf{X} \in \Omega: \operatorname{dist}(\mathbf{X}, \partial \Omega) \geq \epsilon^{\rho} / 2\right\}$. We define $\theta_{k}^{\prime}=\phi_{k}^{\prime} * \eta_{\epsilon^{\rho} / 4}$ and $\theta_{l}=\phi_{l} * \eta_{\epsilon^{\rho}} / 4$. Then $\sum_{k} \theta_{k}^{\prime}+\sum_{l} \theta_{l}=1$ on $\overline{\Omega^{\prime \prime}}, \theta_{k}^{\prime} \in C_{c}^{\infty}\left(B_{\epsilon^{\rho}}\left(\mathbf{X}_{k}\right)\right), \theta_{l} \in C_{c}^{\infty}\left(B_{\epsilon \rho / 2}\left(\mathbf{Y}_{l}\right)\right)$, and $\left|D \theta_{k}^{\prime}\right|,\left|D \theta_{l}\right| \leq C_{23} \epsilon^{-\rho}$.

For $\phi \in C_{c}^{\infty}(\Omega)$, consider $K$ such that $\operatorname{supp}(\phi) \subset \subset K \subset \subset \Omega$ and take $\epsilon<(\operatorname{dist}(K, \partial \Omega))^{(1 / \rho)}$, so that $K \subset \Omega^{\prime \prime}$. Then, using the previously obtained relation $\sum_{l} \theta_{l}+\sum_{k} \theta_{k}^{\prime}=1$ one obtains, writing as above $\mathbf{F}_{\epsilon}^{\mathrm{p}} d X$ for $d \mathbf{F}_{\epsilon}^{\mathrm{p}}$, and $\omega_{l}=B_{\epsilon^{\rho} / 2}\left(\mathbf{Y}_{l}\right)$

$$
\begin{aligned}
& \lim _{\epsilon \rightarrow 0}\left|\int_{\Omega}\left(\mathbf{F}_{\epsilon \alpha}^{\mathrm{p}}-\mathbf{F}_{\epsilon}^{\mathrm{p}}\right) \phi d X\right|=\lim _{\epsilon \rightarrow 0}\left|\int_{K}\left(\mathbf{F}_{\epsilon \alpha}^{\mathrm{p}}-\mathbf{F}_{\epsilon}^{\mathrm{p}}\right) \phi d X\right| \\
&\left.\leq \lim _{\epsilon \rightarrow 0} \sum_{l}\left|\int_{\omega_{l}} \theta_{l}\left(\mathbf{F}_{\epsilon \alpha}^{\mathrm{p}}-\mathbf{F}_{\epsilon}^{\mathrm{p}}\right) \phi d X\right|+\lim _{\epsilon \rightarrow 0} \sum_{k=1}^{N_{\epsilon}} \mid \int_{B_{\epsilon} \rho} \mathbf{X}_{k}\right) \\
& \theta_{k}^{\prime}\left(\mathbf{F}_{\epsilon \alpha}^{\mathrm{p}}-\mathbf{F}_{\epsilon}^{\mathrm{p}}\right) \phi d X \mid
\end{aligned}
$$

In the first term we integrate by parts and use the previously obtained relations $\left|\varphi_{\epsilon \alpha}^{\mathrm{p}}-\varphi_{\epsilon}^{\mathrm{p}}\right| \leq C_{21} \epsilon$ and 


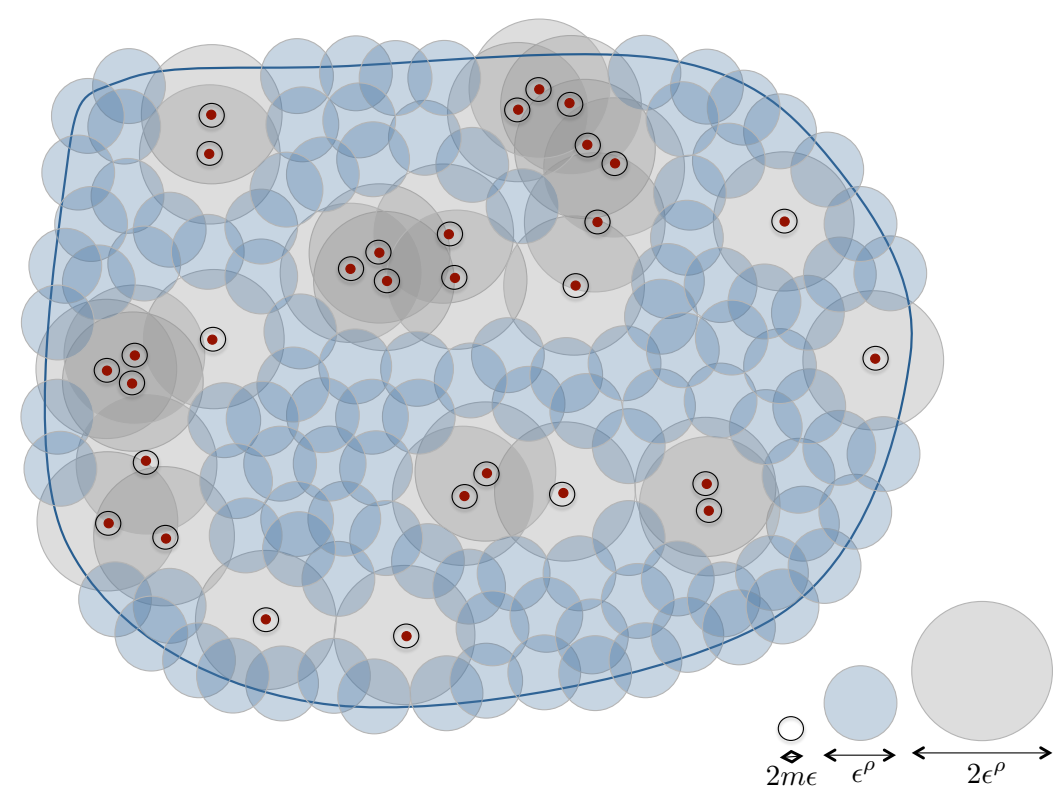

Figure 10: Cover of $\Omega^{\prime \prime}$ with balls $B_{\epsilon} \rho$ centered at the dislocation points $\mathbf{X}_{k}$ (grey balls), and balls $B_{\epsilon^{\rho} / 2}$ with finite overlap (blue balls) that do not overlap with the dislocation cores $B_{m \epsilon}\left(\mathbf{X}_{k}\right)$.

$\left|D \theta_{l}\right| \leq C_{23} \epsilon^{-\rho}$,

$$
\begin{aligned}
\lim _{\epsilon \rightarrow 0} \sum_{l}\left|\int_{\omega_{l}} \theta_{l}\left(\mathbf{F}_{\epsilon \alpha}^{\mathrm{p}}-\mathbf{F}_{\epsilon}^{\mathrm{p}}\right) \phi d X\right| & =\lim _{\epsilon \rightarrow 0} \sum_{l}\left|\int_{\omega_{l}} \theta_{l}\left(D \boldsymbol{\varphi}_{\epsilon \alpha}^{\mathrm{p}}-D \boldsymbol{\varphi}_{\epsilon}^{\mathrm{p}}\right) \phi d X\right| \\
& =\lim _{\epsilon \rightarrow 0} \sum_{l}\left|\int_{\omega_{l}}\left(\boldsymbol{\varphi}_{\epsilon \alpha}^{\mathrm{p}}-\boldsymbol{\varphi}_{\epsilon}^{\mathrm{p}}\right) D\left(\theta_{l} \phi\right) d X\right| \\
& \leq \lim _{\epsilon \rightarrow 0} C_{24} \epsilon\left(\epsilon^{-\rho}\|\phi\|_{L^{\infty}}+\|D \phi\|_{L^{\infty}}\right)=0 .
\end{aligned}
$$

In the second one we use $\left|\theta_{k}^{\prime}\right| \leq 1$ to estimate

$$
\lim _{\epsilon \rightarrow 0} \sum_{k=1}^{N_{\epsilon}}\left|\int_{B_{\epsilon} \rho} \theta_{\left.\mathbf{X}_{k}\right)} \theta_{k}^{\prime}\left(\mathbf{F}_{\epsilon \mathcal{1}}^{\mathrm{p}}-\mathbf{F}_{\epsilon}^{\mathrm{p}}\right) \phi d X\right| \leq\|\phi\|_{L^{\infty}} \lim _{\epsilon \rightarrow 0} \sum_{k=1}^{N_{\epsilon}}\left(\left\|\mathbf{F}_{\epsilon \alpha}^{\mathrm{p}}\right\|_{L^{\infty}}\left|B_{\epsilon^{\rho}}\left(\mathbf{X}_{k}\right)\right|+\left|\mathbf{F}_{\epsilon}^{\mathrm{p}}\right|\left(B_{\epsilon^{\rho}}\left(\mathbf{X}_{k}\right)\right)\right) .
$$

By Lemma 5.4, $\left|\mathbf{F}_{\epsilon}^{\mathrm{p}}\right|\left(B_{\epsilon^{\rho}}\left(\mathbf{X}_{k}\right)\right) \leq C_{25} \epsilon^{2 \rho}$, and recalling that $\left\|\mathbf{F}_{\epsilon \alpha}^{\mathrm{p}}\right\|_{L^{\infty}}$ is bounded, $N_{\epsilon} \leq C / \epsilon$ and $\rho>1 / 2$ we conclude

$$
\left.\lim _{\epsilon \rightarrow 0} \sum_{k=1}^{N_{\epsilon}} \mid \int_{B_{\epsilon} \rho} \mathbf{X}_{k}\right)\left(\theta_{k}^{\prime}\left(\mathbf{F}_{\epsilon \alpha}^{\mathrm{p}}-\mathbf{F}_{\epsilon}^{\mathrm{p}}\right) \phi d X \mid \leq\|\phi\|_{L^{\infty}} \lim _{\epsilon \rightarrow 0} C_{26} N_{\epsilon} \epsilon^{2 \rho}=0 .\right.
$$

As a result $\mathbf{F}_{\epsilon \alpha}^{\mathrm{p}} \stackrel{*}{\rightarrow} \mathbf{F}^{\mathrm{p}}$ in $\mathcal{M}$ and therefore in $L^{\infty}$ as $\epsilon$ tends to zero. Moreover, by the definition of $\varphi_{\epsilon \alpha}^{\mathrm{p}}$, Curl $\mathbf{F}_{\epsilon \alpha}^{\mathrm{p}}=0$ in $\Omega_{2}^{\prime} \backslash \bigcup_{k=1}^{N_{\epsilon}} B_{c \epsilon}\left(\mathbf{X}_{k}\right)$. Furthermore, $\mid$ Curl $\mathbf{F}_{\epsilon \alpha}^{\mathrm{p}} \mid\left(\Omega_{2}^{\prime}\right) \leq N_{\epsilon} N_{s} n \epsilon<\infty$ since each of the $N_{\epsilon}$ dislocation points has at most $N_{s}$ radial jump sets and each of them has a Burgers vector bounded by $n \epsilon$, see Fig. 11. We recall that the determinant of a 2 by 2 tensor can be expressed as the scalar product of two vectors whose Curl and Div (divergence) are controlled by the Curl of the tensor. Indeed,

$$
\operatorname{det} \mathbf{F}=\left(F_{11}, F_{12}\right) \times\left(F_{21}, F_{22}\right)=\left(F_{11}, F_{12}\right) \cdot\left(F_{22},-F_{21}\right),
$$



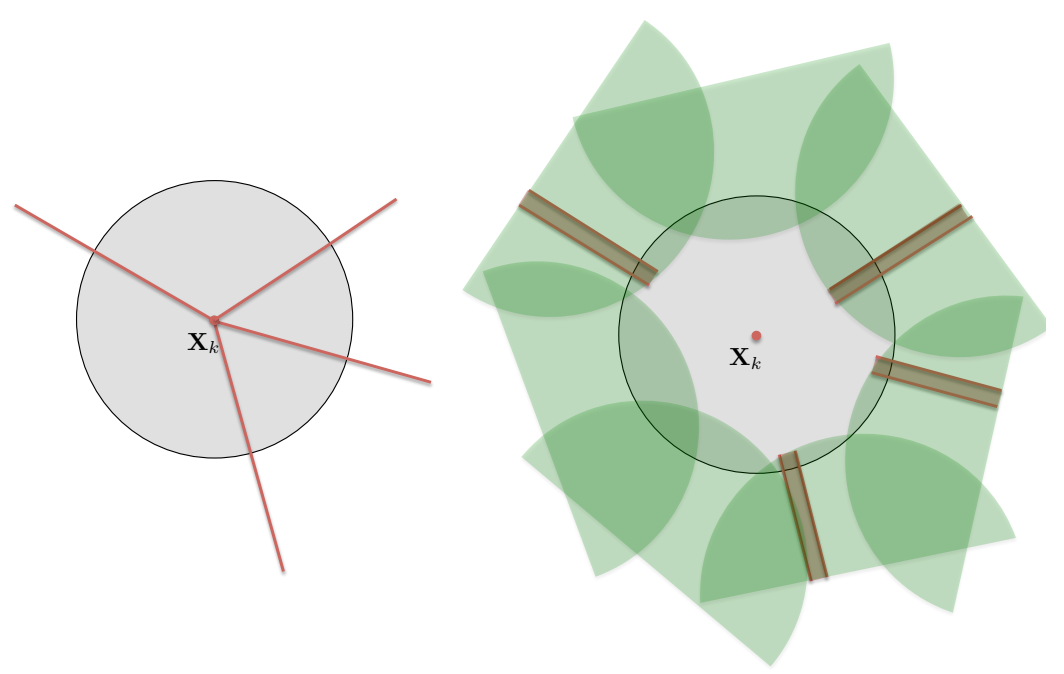

Figure 11: The figure shows the support of $\mathbf{F}_{\epsilon}^{\mathrm{p}}$ in red (left image) and $\mathbf{F}_{\epsilon \alpha}^{\mathrm{p}}$ (right image) near a given dislocation core $B_{c \epsilon}\left(\mathbf{X}_{k}\right)$. The support of $\operatorname{Curl}_{\epsilon}$ is $\mathbf{X}_{k}$, whereas the support of $\operatorname{Curl}_{\epsilon \alpha}^{\mathrm{p}}$ will be $N_{d k} \leq N_{s}$ diffuse dislocations located at the boundary of the domains $B_{\epsilon}$ in which a global definition of $\mathbf{F}_{\epsilon \alpha}^{\mathrm{p}}$ was obtained.

with $\operatorname{Curl}\left(F_{11}, F_{12}\right)=F_{12,1}-F_{11,2}$, and Div $\left(F_{22},-F_{21}\right)=F_{22,1}-F_{21,2}=\operatorname{Curl}\left(F_{21}, F_{22}\right)$. Therefore the div-curl lemma (Evans, 1990) implies $\operatorname{det} \mathbf{F}_{\epsilon \alpha}^{\mathrm{p}} \stackrel{*}{\rightarrow} \operatorname{det} \mathbf{F}^{\mathrm{p}}$ in $L^{\infty}$.

Next we show that $\operatorname{det} \mathbf{F}_{\epsilon \alpha}^{\mathrm{p}} \rightarrow 1$ in $L^{1}$. Since $\operatorname{det} \mathbf{F}_{\epsilon \alpha}^{\mathrm{p}}=1$ on the union of the $\omega_{l}$, and it is zero elsewhere,

$$
\left\|\operatorname{det} \mathbf{F}_{\epsilon \alpha}^{\mathrm{p}}-1\right\|_{L^{1}\left(\Omega_{2}^{\prime}\right)} \leq \sum_{k=1}^{N_{\epsilon}}\left|B_{\epsilon^{\rho}}\left(\mathbf{X}_{k}\right)\right| \leq \pi N_{\epsilon} \epsilon^{2 \rho} \rightarrow 0
$$

since $\rho>1 / 2$. Therefore $\operatorname{det} \mathbf{F}^{\mathrm{p}}=1$ a.e., which is the sought-after result.

In closing we remark that the determinant of the gradient of a $S B V$ function such as $\varphi_{\epsilon}$ can be given a distributional interpretation. This formulation is not used in the proof above, but illustrates the idea behind the statement $\operatorname{det} \mathbf{F}^{\mathrm{p}}=1$. Indeed, in Appendix A we show that if $\varphi_{\epsilon} \in L^{\infty} \cap S B V\left(\Omega ; \mathbb{R}^{2}\right)$ and $\phi \in C_{c}^{\infty}(\Omega)$, the determinant can be interpreted as

$$
\begin{aligned}
\left\langle\operatorname{det} D \boldsymbol{\varphi}_{\epsilon}, \phi\right\rangle & =-\frac{1}{2} \int_{\Omega} \boldsymbol{\varphi}_{\epsilon}^{T} \operatorname{cof} \nabla \boldsymbol{\varphi}_{\epsilon} D \phi d X+\frac{1}{2} \int_{\mathcal{J}}\left(\boldsymbol{\varphi}_{\epsilon} \times \llbracket \boldsymbol{\varphi}_{\epsilon} \rrbracket\right) \cdot(\mathbf{N} \times D \phi) d \mathcal{H}^{1} . \\
& =-\frac{1}{2} \int_{\Omega}\left(\boldsymbol{\varphi}_{\epsilon} \otimes D \phi\right): d \operatorname{cof} D \boldsymbol{\varphi}_{\epsilon},
\end{aligned}
$$

where the precise definition of $\varphi_{\epsilon}$ on $\mathcal{J}$ is irrelevant since $\varphi_{\epsilon}^{+} \times \llbracket \varphi_{\epsilon} \rrbracket=\varphi_{\epsilon}^{-} \times \llbracket \varphi_{\epsilon} \rrbracket$. One can therefore choose for $\varphi_{\epsilon}(\mathbf{X})$ on $\mathbf{X} \in \mathcal{J}$ any convex combination of $\varphi_{\epsilon}^{+}(\mathbf{X})$ and $\varphi_{\epsilon}^{-}(\mathbf{X})$.

\section{Conclusions}

The kinematic assumption $\mathbf{F}=\mathbf{F}^{\mathrm{e}} \mathbf{F}^{\mathrm{p}}$ pertaining to the modeling of elastoplasticity in the setting of large deformations was introduced in the 1960's based on heuristic arguments and has become standard in the continuum mechanics community. However, the lack of a micromechanical justification has raised some skcepticism over the years about its validity, in particular questioning the existence and uniqueness of the multiplicative decomposition. 
In this paper, we provide for the first time, to the best of the authors' knowledge, a rigorous proof of the expression $\mathbf{F}=\mathbf{F}^{\mathrm{e}} \mathbf{F}^{\mathrm{p}}$ for a general elastoplastic deformation of a single crystal, from the independent definitions of $\mathbf{F}, \mathbf{F}^{\mathrm{e}}$ and $\mathbf{F}^{\mathrm{p}}$. In particular, the proof is based on the coarse graining of a mesoscopic description of the deformation, where the dislocations and slip surfaces are individually resolved and the displacement field can be treated as continuous in all the domain except at the surfaces over which dislocations have glided. At such scale, as previously shown by two of the authors (Reina and Conti, 2014), there exists physically-based definitions of the different tensors $\mathbf{F}_{\epsilon}, \mathbf{F}_{\epsilon}^{\mathrm{e}}$ and $\mathbf{F}_{\epsilon}^{\mathrm{p}}$ that are uniquely defined from the microscopic deformation gradient $\varphi_{\epsilon}$ and that do not make use of any multiplicative relationship between them for their definition. Instead, $\varphi_{\epsilon}$ is described in the compatible regions away from the dislocations as the composition of an elastic and plastic deformation, $\varphi_{\epsilon}=\varphi_{\epsilon}^{\mathrm{e}} \circ \varphi_{\epsilon}^{\mathrm{p}}$. The corresponding continuum quantities at the macroscopic scale $\boldsymbol{\varphi}$, $\mathbf{F}, \mathbf{F}^{\mathrm{e}}$ and $\mathbf{F}^{\mathrm{p}}$ are defined respectively as the limit of $\boldsymbol{\varphi}_{\epsilon}, \mathbf{F}_{\epsilon}, \mathbf{F}_{\epsilon}^{\mathrm{e}}$ and $\mathbf{F}_{\epsilon}^{\mathrm{p}}$ as the lattice parameter $\epsilon$ tends to zero in the appropriate topology. We show that the limiting deformation mapping $\boldsymbol{\varphi}$ is indeed continuous, that $\mathbf{F}=\mathbf{F}^{\mathrm{e}} \mathbf{F}^{\mathrm{p}}$ holds in the limit with $\operatorname{det} \mathbf{F}^{\mathrm{p}}=1$, and that Curl $\mathbf{F}^{\mathrm{p}}$ represents the dislocation density tensor when it is expressed in the reference configuration.

The results presented in this paper are of great generality, although limited so far to two dimensional deformations due to the mathematical complexity involved in the proofs. In particular, the assumptions made consist of standard growth conditions for the elastic energy, dislocations separated from each other and from the boundary by a distance of the order of several atomic spacings, a finite variation of the elastic deformation and a precise sequence of slips assumed in the compatible regions of the domain. We remark that this latter assumption is only needed for the proof of $\operatorname{det} \mathbf{F}^{\mathrm{p}}=1$ and it has only been introduced for mathematical simplicity. It is yet to be studied in further detail whether or not some of these assumptions are necessary for the final results to hold.

\section{Appendix A.}

Distributional Curl of a second order tensor. Let $\phi \in C_{c}^{\infty}(\Omega)$, and $\mathbf{F}^{\mathrm{p}}$ smooth. Then using indicial notation and integration by parts,

$$
\int_{\Omega} \phi\left(\operatorname{Curl} \mathbf{F}^{\mathrm{p}}\right)_{i} d X=\int_{\Omega} \phi e_{3 k j}\left(F^{\mathrm{p}}\right)_{i j, k} d X=-\int_{\Omega} e_{3 k j} \phi_{, k}\left(F^{\mathrm{p}}\right)_{i j} d X=-\int_{\Omega}\left(D \phi \times \mathbf{F}^{\mathrm{p}}\right)_{i} d X,
$$

where $e_{i j k}$ is the Levi-Civita symbol and the cross product in the last term is defined row-wise for $\mathbf{F}^{\mathrm{p}}$. The equality of the first and last term is taken as the definition for the distributional Curl.

Determinant of $D \varphi$, with $\varphi \in \mathrm{SBV}$. Let $\phi \in C_{c}^{\infty}(\Omega)$, and $\varphi$ smooth. Then, the weak and strong form of the determinant coincide and read

$$
\int_{\Omega}(\operatorname{det} D \varphi) \phi d X=-\frac{1}{2} \int_{\Omega} \varphi^{T}(\operatorname{cof} D \varphi) D \phi d X=-\frac{1}{2} \int_{\Omega}(\varphi \otimes D \phi):(\operatorname{cof} D \varphi) d X .
$$

Indeed,

$$
\begin{aligned}
\varphi^{T}(\operatorname{cof} D \varphi) D \phi & =\left(\begin{array}{ll}
\varphi_{1} & \varphi_{2}
\end{array}\right)\left(\begin{array}{cc}
\varphi_{2,2} & -\varphi_{2,1} \\
-\varphi_{1,2} & \varphi_{1,1}
\end{array}\right)\left(\begin{array}{c}
\phi_{, 1} \\
\phi_{, 2}
\end{array}\right)=\left(\begin{array}{cc}
\varphi_{1} & \varphi_{2}
\end{array}\right)\left(\begin{array}{c}
\varphi_{2,2} \phi_{, 1}-\varphi_{2,1} \phi_{, 2} \\
-\varphi_{1,2} \phi_{, 1}+\varphi_{1,1} \phi_{, 2}
\end{array}\right) \\
& =\varphi_{1} \varphi_{2,2} \phi_{, 1}-\varphi_{1} \varphi_{2,1} \phi_{, 2}-\varphi_{2} \varphi_{1,2} \phi_{, 1}+\varphi_{2} \varphi_{1,1} \phi_{, 2}
\end{aligned}
$$

Therefore, after integrating by parts,

$$
\begin{aligned}
-\int_{\Omega} \varphi^{T} & (\operatorname{cof} D \varphi) D \phi d X \\
& =\int_{\Omega}\left(\varphi_{1,1} \varphi_{2,2}+\varphi_{1} \varphi_{2,21}-\varphi_{1,2} \varphi_{2,1}-\varphi_{1} \varphi_{2,12}-\varphi_{2,1} \varphi_{1,2}-\varphi_{2} \varphi_{1,21}+\varphi_{2,2} \varphi_{1,1}+\varphi_{2} \varphi_{1,12}\right) \phi d X \\
& =2 \int_{\Omega}\left(\varphi_{1,1} \varphi_{2,2}-\varphi_{1,2} \varphi_{2,1}\right) \phi d X=2 \int_{\Omega}(\operatorname{det} D \varphi) \phi d X
\end{aligned}
$$


For $\varphi \in S B V, \operatorname{cof} D \varphi=\operatorname{cof} \nabla \varphi \mathcal{L}^{2}+\operatorname{cof}(\llbracket \varphi \rrbracket \otimes \mathbf{N}) \mathcal{H}^{1}\lfloor\mathcal{J}$, with

$$
\begin{aligned}
& \operatorname{cof}(\llbracket \varphi \rrbracket \otimes N)=\operatorname{cof}\left(\begin{array}{cc}
\llbracket \varphi_{1} \rrbracket N_{1} & \llbracket \varphi_{1} \rrbracket N_{2} \\
\llbracket \varphi_{2} \rrbracket N_{1} & \llbracket \varphi_{2} \rrbracket N_{2}
\end{array}\right)=\left(\begin{array}{cc}
\llbracket \varphi_{2} \rrbracket N_{2} & -\llbracket \varphi_{2} \rrbracket N_{1} \\
-\llbracket \varphi_{1} \rrbracket N_{2} & \llbracket \varphi_{1} \rrbracket N_{1}
\end{array}\right) \\
& =\left(\begin{array}{c}
-\llbracket \varphi_{2} \rrbracket \\
\llbracket \varphi_{1} \rrbracket
\end{array}\right) \otimes\left(\begin{array}{c}
-N_{2} \\
N_{1}
\end{array}\right)=\llbracket \varphi \rrbracket^{\perp} \otimes \mathbf{N}^{\perp}, \\
& \boldsymbol{\varphi}^{T} \operatorname{cof}(\llbracket \boldsymbol{\varphi} \rrbracket \otimes N) D \phi=\left(\boldsymbol{\varphi} \cdot \llbracket \boldsymbol{\varphi} \rrbracket^{\perp}\right)\left(\mathbf{N}^{\perp} \cdot D \phi\right) \\
& =\left(\left(\begin{array}{c}
\varphi_{1} \\
\varphi_{2}
\end{array}\right) \cdot\left(\begin{array}{c}
-\llbracket \varphi_{2} \rrbracket \\
\llbracket \varphi_{1} \rrbracket
\end{array}\right)\right)\left(\left(\begin{array}{c}
-N_{2} \\
N_{1}
\end{array}\right) \cdot\left(\begin{array}{c}
\phi_{, 1} \\
\phi_{, 2}
\end{array}\right)\right) \\
& =(-\varphi \times \llbracket \varphi \rrbracket) \cdot(\mathbf{N} \times D \phi),
\end{aligned}
$$

therefore giving (6.64).

\section{Acknowledgements}

C. Reina thanks Prof. Arash Yavari for sharing his knowledge on the origins of the multiplicative decomposition. C. Reina acknowledges the NSF grant CMMI - 1401537. S. Conti acknowledges support from the Deutsche Forschungsgemeinschaft through SFB 1060 "The Mathematics of emergent effects", project A5. All the authors further acknowledge support from the Hausdorff Center for Mathematics.

\section{References}

A. Acharya and J. L. Bassani. Lattice incompatibility and a gradient theory of crystal plasticity. Journal of the Mechanics and Physics of Solids, 48:1565-1595, 2000.

R. A. Adams and J. J. F. Fournier. Sobolev spaces, volume 140 of Pure and Applied Mathematics (Amsterdam). Elsevier/Academic Press, Amsterdam, second edition, 2003. ISBN 0-12-044143-8.

E. C Aifantis. Exploring the applicability of gradient elasticity to certain micro/nano reliability problems. Microsystem Technologies, 15(1):109-115, 2009.

R. Alicandro, L. De Luca, A. Garroni, and M. Ponsiglione. Dynamics of discrete screw dislocations on glide directions. preprint CVGMT, 2015.

L. Ambrosio, N. Fusco, and D. Pallara. Functions of Bounded Variation and Free Discontinuity Problems. Oxford Science Publications, 2000.

D. J. Bammann and G. C. Johnson. On the kinematics of finite-deformation plasticity. Acta Mechanica, 70(1-4):1-13, 1987.

N. R Barton, A. Arsenlis, and J. Marian. A polycrystal plasticity model of strain localization in irradiated iron. Journal of the Mechanics and Physics of Solids, 61(2):341-351, 2013.

B. A. Bilby. Continuous distributions of dislocations: a new application of the methods of non-riemannian geometry. Proceedings of the Royal Society of London. Series A, Mathematical and PhysicalSciences, 231:263-273, 1955.

B. A. Bilby, L. R. T. Gardner, and A. N. Stroh. Continuous distributions of dislocations and the theory of plasticity. In 9th International Congress in Applied Mechanics, volume 8, pages 35-44, 1957.

T. Blass, I. Fonseca, G. Leoni, and M. Morandotti. Dynamics for systems of screw dislocations. SIAM J. Appl. Math., 75: 393-419, 2015.

J. Casey. A convenient form of the multiplicative decomposition of the deformation gradient. Mathematics and Mechanics of Solids, page 1081286515598662, 2015.

J. Casey and P. M. Naghdi. A remark on the use of the decomposition $F=F_{e} F_{p}$ in plasticity. Journal of Applied Mechanics, 47:672-675, 1980 .

J. Casey and P. M. Naghdi. A prescription for the identification of finite plastic strain. International Journal of Engineering Science, 30:1257-1278, 1992 .

P. Cermelli and M. E. Gurtin. On the characterization of geometrically necessary dislocations in finite plasticity. Journal of the Mechanics and Physics of Solids, 49:1539-1568, 2001.

J. D. Clayton, C. S. Hartley, and D. L. McDowell. The missing term in the decomposition of finite deformation. International Journal of Plasticity, 52:5176, 2014.

R. J. Clifton. On the equivalence of $\mathrm{F}^{e} \mathrm{~F}^{p}$ and $\mathrm{F}^{p} \mathrm{~F}^{e}$. Journal of Applied Mechanics, 39:287-230, 1972.

S. Conti and M. Ortiz. Dislocation microstructures and the effective behavior of single crystals. Arch. Rat. Mech. Anal., 176: 103-147, 2005.

S. Conti and F. Theil. Single-slip elastoplastic microstructures. Arch. Rat. Mech. Anal., 178:125-148, 2005.

S. Conti, G. Dolzmann, and C. Kreisbeck. Asymptotic behavior of crystal plasticity with one slip system in the limit of rigid elasticity. SIAM J. Math. Anal., 43:2337-2353, 2011. 
Y. F. Dafalias. Issues on the constitutive formulation at large elastoplastic deformations, part 1: Kinematics. Acta Mechanica, 69:119-138, 1987.

Dassault Systèmes. Abaqus 6.12 theory manual. Dassault Systèmes Simulia Corp., Providence, Rhode Island, 2012.

L. Davison. Kinematics of finite elastoplastic deformation. Mechanics of materials, 21(1):73-88, 1995.

L. De Luca, A. Garroni, and M. Ponsiglione. $\Gamma$-convergence analysis of systems of edge dislocations: the self energy regime. Archive for Rational Mechanics and Analysis, 206(3):885-910, 2012.

G. Del Piero and D. R. Owen. Structured deformations of continua. Arch. Rational Mech. Anal., 124:99-155, 1993.

G. Del Piero and D. R. Owen. Multiscale modeling in continuum mechanics and structured deformations. Springer, 2004.

L. Deseri and D. R. Owen. Invertible structured deformations and the geometry of multiple slip in single crystals. International Journal of Plasticity, 18:833-849, 2002.

C. Eckart. The thermodynamics of irreversible processes. iv. the theory of elasticity and anelasticity. Physical Review, $73(4)$ : 373,1948 .

J. D. Eshelby. The continuum theory of lattice defects. Solid State Physics, 3:79-144, 1956.

L. C Evans. Weak convergence methods for nonlinear partial differential equations. Number 74. American Mathematical Soc., 1990 .

L. C. Evans and R. F. Gariepy. Measure Theory and Fine Properties of Functions. CRC Press, 1991.

N. Fox. A continuum theory of dislocations for single crystals. Journal of the Institute of Mathematics and its applications, 2: $285-288,1966$.

A. Garroni, G. Leoni, and M. Ponsiglione. Gradient theory for plasticity via homogenization of discrete dislocations. Journal of the European Mathematical Society, 12:1231-1266, 2010.

J. M. Gerken and P. R. Dawson. A crystal plasticity model that incorporates stresses and strains due to slip gradients. Journal of the Mechanics and Physics of Solids, 56:1651-1672, 2008.

A. E. Green and P. M. Naghdi. Some remarks on elastic-plastic deformation at finite strain. International Journal of Engineering Science, 9:1219-1229, 1971.

K. Hackl, S. Heinz, and A. Mielke. A model for the evolution of laminates in finite-strain elastoplasticity. ZAMM-Journal of Applied Mathematics and Mechanics/Zeitschrift für Angewandte Mathematik und Mechanik, 92(11-12):888-909, 2012.

D. L. Henann and L. Anand. A large deformation theory for rate-dependent elastic-plastic materials with combined isotropic and kinematic hardening. International Journal of Plasticity, 25(10):1833-1878, 2009.

E. Kröner. Allgemeine Kontinuumstheorie der Versetzungen und Eigenspannungen. Archive for rational mechanics and analysis, 4:273-334, 1959 .

E. H. Lee and D. T. Liu. Finite strain elastic-plastic theory with application to plane wave analysis. Journal of Applied Physics, 38:19-27, 1967.

A. Lion. Constitutive modelling in finite thermoviscoplasticity: a physical approach based on nonlinear rheological models. International Journal of Plasticity, 16:469-494, 2000.

V. A. Lubarda. Duality in constitutive formulation of finite-strain elastoplasticity based on $F=F_{e} F_{p}$ and $F=F_{p} F_{e}$ decompositions. International Journal of Plasticity, 15:1277-1290, 1999.

V. A. Lubarda and E. H. Lee. A correct definition of elastic and plastic deformation and its computational significance. Journal of Applied Mechanics, 48:35-40, 1981.

S. Luckhaus and L. Mugnai. On a mesoscopic many-body Hamiltonian describing elastic shears and dislocations. Continuum Mechanics And Thermodynamics, 22:251-290, 2010.

A. Mainik and A. Mielke. Global existence for rate-independent gradient plasticity at finite strain. J. Nonlinear Sci., 19(3): 221-248, 2009.

J. Mandel. Equations constitutives et directeurs dans les milieux plastiques et viscoplastiques. International Journal of Solids and Structures, 9:725-740, 1973.

A. Mielke and S. Müller. Lower semicontinuity and existence of minimizers in incremental finite-strain elastoplasticity. $Z A M M$ Journal of Applied Mathematics and Mechanics/Zeitschrift für Angewandte Mathematik und Mechanik, 86:233-250, 2006.

A. Mielke and U. Stefanelli. Linearized plasticity is the evolutionary C-limit of finite plasticity. J. Eur. Math. Soc. (JEMS), 15(3):923-948, 2013.

S. Müller, L. Scardia, and C. I. Zeppieri. Geometric rigidity for incompatible fields and an application to strain-gradient plasticity. Indiana University Mathematics Journal, 63:1365-1396, 2015.

P. M. Naghdi. A critical review of the state of finite plasticity. Journal of Applied Mathematics and Physics, 41:315-675, 1990.

S. Nemat-Nasser. Decomposition of strain measures and their rates in finite deformation elastoplasticity. International Journal of Solids and Structures, 15:155-166, 1979.

S. Nemat-Nasser. Plasticity: a treatise on finite deformation of heterogeneous inelastic materials. Cambridge University Press, 2004.

M. Ortiz and E. A. Repetto. Nonconvex energy minimization and dislocation structures in ductile single crystals. Journal of the Mechanics and Physics of Solids, 47:397-462, 1999.

S. T. Pantelides. First principles mesoscopic dynamics in heterogeneous materials. Journal of Applied Physics, 3264:155-166, 1994.

C. Reina and S. Conti. Kinematic description of crystal plasticity in the finite kinematic framework: A micromechanical understanding of $\mathrm{F}=\mathrm{F}^{e} \mathrm{~F}^{p}$. Journal of the Mechanics and Physics of Solids, 67:40-61, 2014.

J. R. Rice. Inelastic constitutive relations for solids: an internal-variable theory and its application to metal plasticity. Journal of the Mechanics and Physics of Solids, 19:433-455, 1971.

S. Sadik and A. Yavari. On the origins of the idea of the multiplicative decomposition of the deformation gradient. Mathematics and Mechanics of Solids, pages 1-2, 2015. 
L. Scardia and C. I. Zeppieri. Line-tension model for plasticity as the $\Gamma$-limit of a nonlinear dislocation energy. SIAM J. Math. Anal., 44:2372-2400, 2012

L. Scardia, R. Peerlings, M. A. Peletier, and M. G. D. Geers. Mechanics of dislocation pile-ups: A unification of scaling regimes. Journal of the Mechanics and Physics of Solids, 70:42-61, 2014.

J. C. Simo. A framework for finite strain elastoplasticity based on maximum plastic dissipation and the multiplicative decomposition. Part II: Computational aspects. Computer Methods in Applied Mechanics and Engineering, 68(1):1-31, 1988.

C. Truesdell. Hypo-elasticity. Journal of Rational Mechanics and Analysis, 4(1):83-131, 1955.

K. Y. Volokh. An approach to elastoplasticity at large deformations. European Journal of Mechanics-A/Solids, 39:153-162, 2013

J. R. Willis. Second-order effects of dislocations in anisotropic crystals. International Journal of Engineering Science, 5: 171-190, 1967.

H. Xiao, O. T. Bruhns, and A. Meyers. Elastoplasticity beyond small deformations. Acta Mechanica, 182(1-2):31-111, 2006.

H. M. Zbib. On the mechanics of large inelastic deformations: kinematics and constitutive modeling. Acta Mechanica, 96: 119-138, 1993. 